\title{
Mitteilungen aus dem Laboratorium für Geophysik der Carnegie-Institution in Washington. Nr. 25. \\ Das ternäre System: Calciumoxyd-Aluminiumoxyd-Silicium-2-oxyd.
}

\author{
Von G. A. Rankin. \\ Optische Untersuchungen von Fred. E. Wright. ${ }^{1}$ \\ Mit 19 Figuren im Text.
}

\section{Einleitung.}

Die Stabilitätsbeziehungen des ternären Systemes: CalciumoxydAluminiumoxyd-Silicium-2-oxyd festzustellen, ist nicht nur aus rein wissenschaftlichen Gesichtspunkten von Bedeutung, sondern auch wegen der Beziehungen mancher auf diese Weise aufgefundenen Tatsachen zu geologischen Fragen, sowie zu dem Problem der Natur der Portland-Zement-Klinker. Es sind bereits viele Arbeiten über diesen Gegenstand veröffentlicht worden, in der Hauptsache ist aber das bekannt gewordene Material noch lückenhaft und nicht ausreichend, die großen allgemeinen Fragen, um die es sich handelt, vollständig zu lösen. Die vorliegende Untersuchung ging darauf hinaus, das fragliche System ziemlich vollständig zu behandeln, um seine Gleichgewichtsbeziehungen sicherzustellen. Zu diesem Zweck haben wir alle möglichen Verbindungen, die sich in den trockenen Schmelzen der drei Oxyde: Calciumoxyd, Aluminiumoxyd, Silicium2-oxyd bilden, bestimmt, wobei wir ins besondere diejenigen studierten, die in Gegenwart von Flüssigkeit stabil sind; dies bedingt die Messung der Schmelzpunkte oder Dissoziationstemperaturen und die Bestimmung der invarianten Punkte, der Grenzkurven (monovariante Systeme) und der Stabilitätsfelder (divariante Systeme) bei den verschiedenen Verbindungen.

Auf den folgenden Seiten legen wir eine Gesamtübersicht über die durchgeführten Untersuchungen vor; wir glauben, es ist der erste durchgreifende Versuch, alle Verbindungen - binäre sowohl wie ternäre - festzustellen, die nur aus Calciumoxyd, Aluminium-

${ }^{1}$ Aus dem Manuskript ins Deutsche übertragen von J. Kopper-Berlin. 
oxyd und Sicilium-2-oxyd bestehen, sowie die wechselseitigen Beziehungen dieser Verbindungen, von denen viele natürlich bereits synthetisch von anderen dargestellt worden sind. Die erhaltenen Daten werden bei einer Besprechung über Natur und Konstitution der Portland-Zement-Klinker und über die Bildung gewisser natürlicher Mineralien aus dem Magma verwendet.

Drei Arbeiten über dieses System sind bereits aus diesem Laboratorium hervorgegangen. Die ersten beiden beschäftigten sich mit den binären Systemen ${ }^{1}$, während in der dritten ${ }^{2}$ vorläufig die ternären Quintupelpunkte und Grenzkurven festgelegt und die erhaltenen Beziehungen zu einer Besprechung der Konstitution der Portland-Zement-Klinker verwendet wurde. In der vorliegenden Arbeit können wir die Lage der eutektischen und Quintupelpunkte sowie der Grenzkurven näher angeben und ebenso die entsprechenden Temperaturen mitteilen. Wegen der großen Anzahl von Daten ist es nicht möglich, in einer Arbeit von dieser Art mehr als die Mittelwerte, die aus einer großen Anzahl von Bestimmungen der verschiedenen Punkte erhalten wurden, mitzuteilen.

\section{Die Komponenten und die binären Systeme.}

Es wird zweckmäßig sein, eine kurze Übersicht über die bei der Untersuchung der binären Systeme erhaltenen Resultate zu geben, einerseits damit wir die Schlüsse klar vor uns haben, auf denen diese Untersuchung begründet ist, und andererseits weil verbesserte $\mathrm{Me}$ thoden zur Schmelzpunktbestimmung für einige Punkte neue Temperaturwerte geliefert haben.

Die drei Komponenten: Calciumoxyd, Aluminiumoxyd, Silicium-2-oxyd.

Calciumoxyd, CaO. Der Schmelzpunkt von reinem Calciumoxyd ist neuerdings von $\mathrm{K}_{\mathrm{ANOLT}} \mathrm{T}^{\mathbf{3}} \mathrm{zu} 2570^{\circ}$ bestimmt worden. KanoLT benutzte einen Graphitofen und wandte besondere Vorsichtsmaßregeln an, um eine Verunreinigung der Schmelze zu verhindern. Es

1 Die Kalk-Silicium-2-oxydreihe der Mineralien, A. L. DAY, E. S. SHEPHERD, F. E. WRIG HT, Amer. Journ. Sci. (4) 22 (1906), 265. - Die binären Systeme von Aluminiumoxyd mit Silicium-2-oxyd, Kalk und Magnesia, E. S. SHEPHERD, G. A. RANkIN, F. E. WRIGHT, Amer. Journ. Sci. (4) 28 (1909), 293.

2 Vorläufiger Bericht über das ternäre System $\mathrm{CaO}-\mathrm{Al}_{2} \mathrm{O}_{3}-\mathrm{SiO}_{2}$, eine Untersuchung über die Konstitution der Portland-Zement-Klinker, E. S. SHEPHerd, G. A. Rankin, F. E. Wriatr, J. Ind. Eng. Chem. 3 (1911), 211-221.

${ }^{3}$ J. Wash. Acad. Sci. 3 (1913), 315. 
ist schwierig festzustellen, welche Genauigkeit dieser Messung zukommt, jedenfalls ist sie die beste, die zurzeit vorhanden ist. Bisher sind keine Andeutungen über die Existenz einer zweiten Form bekannt geworden.

Gut entwickelte Kristalle von Kalk wurden nur aus verhältnismäßig großen Schmelzen von reinem Calciumoxyd erhalten. Die Kristalle sind farblos oder schwach gelb, durchsichtig, und wurden nur in unveränderten Würfeln des isometrischen Systemes beobachtet. Die Spaltbarkeit ist vollkommen nach dem Würfel (100). Die Kristalle sind isotrop; gelegentlich zeigen die größeren Individuen optische Anomalien, die wahrscheinlich auf innere Spannungen zurückzuführen sind. Bei den Präparaten erscheint das freie Caliciumoxyd gewöhnlich in feinen isotropen Körnern, die meist abgerundeten Umriß und sehr hohes Brechungsvermögen besitzen (etwa 1.83). Die Körner sind leicht zu erkennen, selbst wenn sie nur einen Durchmesser von wenigen Tausendsteln Millimeter haben.

Aluminiumoxyd, $\mathrm{Al}_{2} \mathrm{O}_{3} \cdot{ }^{1} \quad \mathrm{~K} \ddot{n}$ stlicher Korund. Der Schmelzpunkt von Aluminiumoxyd ist von $\mathrm{KaNOLT}^{2}$ zu $2050^{\circ}$ bestimmt worden. Die optischen Eigenschaften des künstlichen Korunds stimmen gut mit denen des natürlichen Minerales überein. Aus einer Schmelze von reinem Aluminiumoxyd kristallisierte Körner zeigen nur selten Kristallbegrenzung; sie sind abgerundet und haben einen Durchmesser von weniger als $0.05 \mathrm{~mm}$. Kleine Lufthöhlungen in den Körnern sind reichlich und charakteristisch. Die Härte ist 9. Die Brechungsindizes sind $\omega_{N a}=1.768 \pm 0.003: \varepsilon_{N a}=1.760 \pm 0.003$; die Doppelbrechung ist ziemlich schwach, etwa 0.009. Die Kristalle sind einachsig und optisch negativ. Aus Gemischen kristallisiert es gewöhnlich in dünnen, scharf begrenzten hexagonalen Platten.

Silicium-2-oxyd, $\mathrm{SiO}_{2}$. Eine ausgedehnte Untersuchung über die Stabilitätsbeziehungen der verschiedenen Kristallformen von $\mathrm{SiO}_{2}$ ist in diesem Laboratorium von C. N. FenNer ${ }^{3}$ ausgeführt worden. Die Ergebnisse dieser Arbeit sind kurz folgende:

1 Eine zweite Form von Aluminiumoxyd ist neuerdings bei Arbeiten über das ternäre System $\mathrm{CaO}-\mathrm{AlO}-\mathrm{MgO}$ aufgefunden worden. Eine vollständige Beschreibung derselben soll erfolgen, wenn diese Untersuchung veröffentlicht wird.

2 J. Wash. Acad. Sci. 3 (1913), 315.

${ }^{3}$ Amer. Journ. Sci. (4) 36 (1913), 331. 


\begin{tabular}{|c|c|c|}
\hline Umwandlung & Temperatur & Bemerkungen \\
\hline 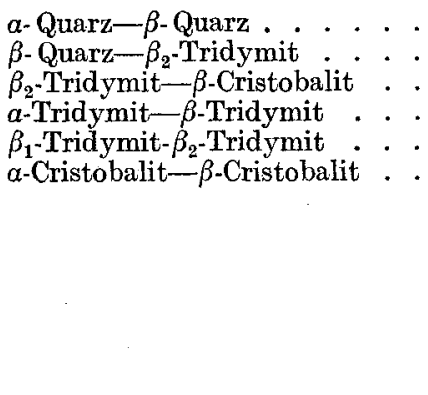 & $\begin{array}{l}575^{\circ} \\
870^{\circ} \pm 10^{0} \\
1470^{\circ} \pm 10^{0} \\
117^{\circ} \\
163^{\circ} \\
270^{\circ}-275^{\circ}\end{array}$ & $\begin{array}{l}\text { Schnell, umkehrbar. } \\
\text { Sehr träge, umkehrbar. } \\
\text { Sehr träge, umkehrbar. } \\
\text { Schnell, umkehrbar. } \\
\text { Schnell, umkehrbar. } \\
\text { Schnell, umkehrbar; Temp. } \\
\text { hängt von der Vorbehand- } \\
\text { lung ab. In Gegenwart } \\
\text { eines Flußmittels bei ver- } \\
\text { hältnismäßig niedrigen Tem- } \\
\text { peraturen geht er langsam } \\
\text { in Chalcedon, Tridymit oder } \\
\text { Quarz über. }\end{array}$ \\
\hline
\end{tabular}

Der Schmelzpunkt von Cristobalit liegt bei etwa $1625^{\circ}$, der des metastabilen Quarzes bei $1470^{\circ}$. Neuere Untersuchungen von N. L. BoweN ${ }^{1}$ in diesem Laboratorium haben gezeigt, daß die Schmelztemperatur von Cristobalit unter gewissen Bedingungen beträchtlich über $1625^{\circ}$ liegt, und daß wahrscheinlich die Schmelztemperatur, ebenso wie die Umwandlungstemperatur wechselnd ist, so daß sich Cristobalit in dieser Beziehung wie die feste Lösung zweier Komponenten verhält.

Die bei niedrigen Temperaturen auftretenden Formen sind: $\alpha$-Quarz, $\alpha$-Tridymit, $\alpha$-Cristobalit und Chalcedon.

Die Kristalle von $\alpha-$ Quarz aus Schmelzen von Natriumwolframat haben nur selten eine Länge von mehr als $0.1 \mathrm{~mm}$; sie sind doppelt vom Einheitsprisma und Rhomboeder als Grenzflächen begrenzt. Die Kristalle sind hexagonal und gleichen in jeder Beziehung den natürlichen Quarzkristallen. Brechungsindizes für Natriumlicht $\omega_{N a}=1.544 ; \varepsilon_{N a}=1.553 ;$ Doppelbrechung etwa 0.01 . Die Kristalle sind einachsig und optisch positiv.

$\alpha$-Tridymit-Kristalle treten in kryptokristallinen Aggregaten von hexagonaler Form auf. Die Brechungsindizes sind ungefähr $\alpha_{N a}=1.469, \quad \gamma_{N a}=1.473$. Die Doppelbrechung ist außerordentlich schwach. Winkel der optjschen Achsen groß. Die $\alpha$-Form ist wahrscheinlich rhombisch, während die oberhalb $117^{\circ}$ stabile $\beta$-Form hexagonal ist.

a-Cristobalit tritt gleichfalls in kryptokristallinen Aggregaten auf. Brechungsindizes: $\alpha_{N a}=1.484, \gamma_{N a}=1.487$; Doppelbrechung schwach. Das Kristallsystem ist wahrscheinlich tetragonal oder rhombisch. Die $\beta$-Form kristallisiert isometrisch.

1 Amer. Journ. (4) 38 (1914), 218. 
Chalcedon wurde künstlich nicht erhalten; die optischen Eigenschaften des natửrlichen Minerales finden sich in den Handbüchern der mikroskopischen Petrographie.

\section{Das System Calciumoxyd-Silicium-2-oxyd.}

Wenn man Calciumoxyd und Silicium-2-oxyd miteinander erhitzt, so können sich vier verschiedene Verbindungen bilden:

1. Das Metasilicat $\mathrm{CaO} \cdot \mathrm{SiO}_{2}$;

2. die Verbindung $3 \mathrm{CaO} \cdot 2 \mathrm{SiO}_{2}$;

3. das Orthosilicat $2 \mathrm{CaO} \cdot \mathrm{SiO}_{2}$;

4. das Trisilicat $3 \mathrm{CaO} \cdot \mathrm{SiO}_{2}$.

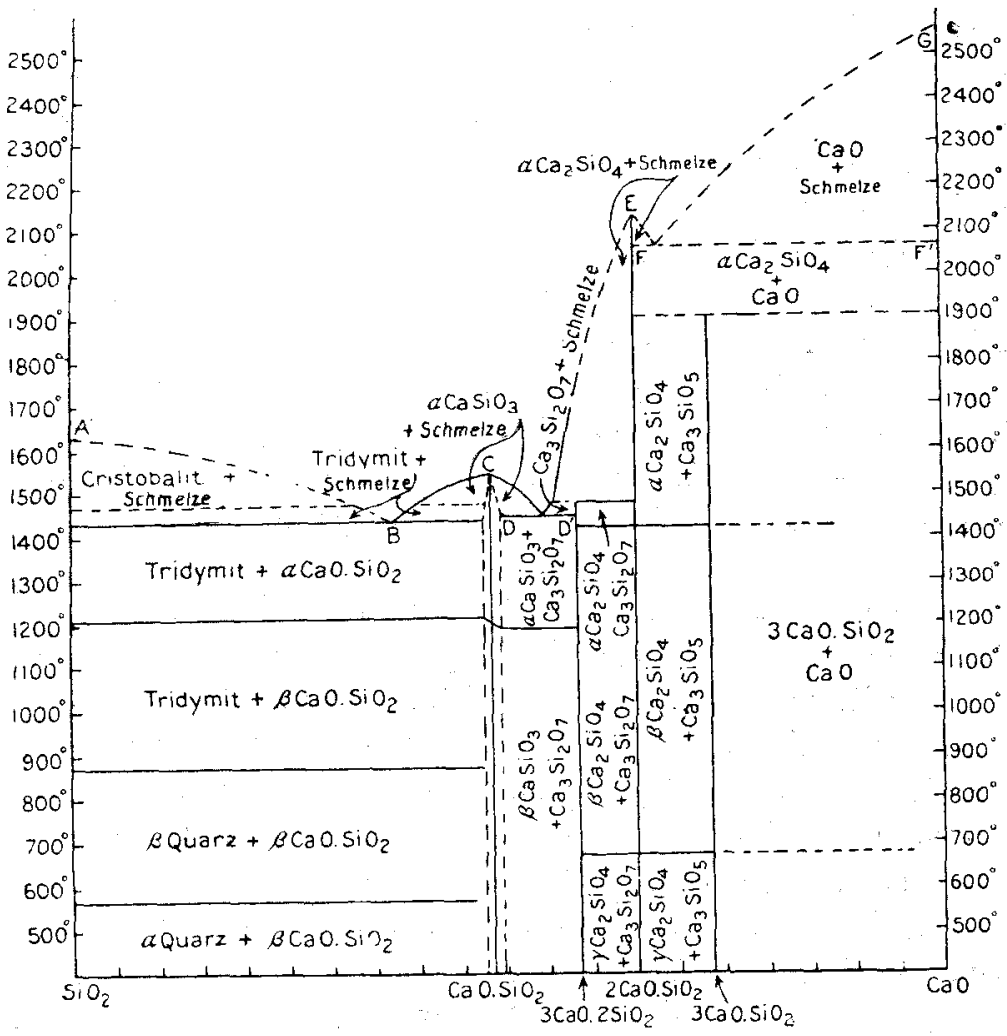

Fig. 1. Konzentrations-Temperatur-Diagramm des Systemes $\mathrm{CaO}-\mathrm{SiO}_{2}$.

Mehrere dieser Verbindungen treten in mehr als einer Kristallform auf. Das Temperatur-Konzentrations-Diagramm dieses binären Systems ist in Fig. 1 dargestellt. 
(1) Calcium-meta-silicat $\mathrm{CaO} \cdot \mathrm{SiO}_{2}\left(\mathrm{CaO}=48.3 \%, \mathrm{SiO}_{2}=\right.$ $51.7 \%)^{1}$ Diese Verbindung tritt in zwei verschiedenen Formen auf.

1. Als Mineral Wollastonit $\left(\beta-\mathrm{CaO} \cdot \mathrm{SiO}_{2}\right)$, welches besonders in kontaktmetamorphischen Aureolen von Kalkstein gefunden wird und bis $1200^{\circ}$, dem umkehrbaren Umwandlungspunkt, stabil ist;

2. als Pseudowollastonit $\left(\alpha-\mathrm{CaO} \cdot \mathrm{SiO}_{2}\right)$, das in der Natur bisher niemals beobachtet worden ist, sondern nur in künstlichen Schmelzen und Schlacken; es ist die stabile Form zwischen $1200^{\circ}$ und dem Schmelzpunkt $1540^{\circ} \pm 2$. Pseudowollastonit nimmt etwa $2 \%$ Calciumoxyd oder Silicium-2-oxyd in fester Lösung auf; derartige Zusätze verändern den Umwandlungspunkt; der Kalk erniedrigt ihn auf $1190^{\circ}$, während das Silicium-2-oxyd ihn auf $1210^{\circ}$ erhöht. Das Eutektikum zwischen $\alpha$-CaO $\cdot \mathrm{SiO}_{2}$ und Tridymit (der stabilen Form von Silicium-2-oxyd bei der eutektischen Temperatur) hat die Zusammensetzung $\mathrm{CaO}=37 \%, \mathrm{SiO}_{2}=63 \%$ in Gewichtsprozenten; seine Schmelztemperatur ist $1436^{\circ} \pm 2^{\circ}$. Die anderen allgemeinen Beziehungen ergeben sich aus der Zeichnung.

$\alpha-\mathrm{CaO} \cdot \mathrm{SiO}_{2}$. Kristallisiert in gleichmäßigen Körnern, ohne entschiedene Kristallbegrenzung; polysynthetische Zwillingsbildung ist nicht ungewöhnlich und deutet, zusammen mit den optischen Eigenschaften, darauf hin, daß das Kristallsystem wahrscheinlich monoklin ist. Brechungsindizes für Natriumlicht: $\gamma_{N a}=1.654 \pm 0.002$; $\alpha_{N a}=1.610 \pm 0.002$; Doppelbrechung stark, $\gamma$ - $\alpha$ etwa 0.041 ; Winkel der optischen Achsen klein, fast einachsig; optischer Charakter: positiv.

$\beta$ - $\mathrm{CaO} \cdot \mathrm{SiO}_{2}$ tritt in lattenförmigen Individuen auf, die dem natürlichen Mineral Wollastonit in jeder Beziehung ähnlich sind. Spaltbarkeit, parallel zur Längsrichtung der Latten und Fasern, ist gut ausgebildet. Kristallsystem monoklin; Brechungsindizes $\gamma_{N a}=1.631 \pm 0.002, \quad \beta_{N a}=1.629 \pm 0.002, \quad \alpha_{N a}=1.616 \pm 0.002$, mittlere Doppelbrechung, $\gamma-\alpha$ etwa gleich 0.015; Auslöschung parallel zur Längsrichtung; Winkel der optischen Achsen $2 E$ etwa 70 . Optischer Charakter: negativ; Ebene der optischen Achsen: normal zu den Spaltlinien.

(2.) Die Verbindung $3 \mathrm{CaO} \cdot 2 \mathrm{SiO}_{2}\left(\mathrm{CaO}=58.2 \%, \quad \mathrm{SiO}_{2}=\right.$ $41.8 \%$ ) spaltet sich in $2 \mathrm{CaO} \cdot \mathrm{SiO}_{2}$ und Flüssigkeit bei $1475^{\circ} \pm 5^{\circ}$ und zeigt demnach keinen wahren Schmelzpunkt. In der binären

1 Alle Zusammensetzungen sind in Gewichtsprozenten ausgedrückt, wenn nichts anderes angegeben ist. 
Reihe tritt sie als primäre Phase auf der Flüssigkeitslinie nur bei Gemischen zwischen 54.5 und 55.5\% Calciumoxyd auf. Sie wird am besten gebildet, wenn man ein Glas der Zusammensetzung $3 \mathrm{CaO} \cdot 2 \mathrm{SiO}_{2}$ etwas unter $1475^{\circ}$ hält, bis die Kristallisation eintritt.

Diese Verbindung kristallisiert in abgerundeten, unregelmäßigen Körnern ohne bestimmte Kristallbegrenzung und ohne deutliche Spaltbarkeit. Das Kristallsystem ist wahrscheinlich rhombisch. Die Brechungsindizes sind $\gamma_{N a}=1.650 \pm 0.002, \alpha_{N a}=1.641 \pm 0.002$; die Doppelbrechung ist ziemlich schwach, $\gamma-\alpha$ etwa 0.01 ; Winkel der optischen Achsen groß; optischer Charakter: positiv.

Man dachte zuerst, daß diese Substanz reiner Åkermanit wäre, dessen Formel nach Vogt $4 \mathrm{CaO} \cdot 3 \mathrm{SiO}_{2}$ ist; aber dies Mineral kristallisiert tetragonal und einachsig mit Brechungsindizes $\gamma$ von etwa 1.640, $\alpha=$ etwa 1.635. Die Gegenwart von Magnesia in Voats Âkermanit mag über die beobachteten Unterschiede im optischen Verhalten der beiden Stoffe Aufschluß geben; und wenn dies der Fall ist, so sollte die Formel für reinen Åkermanit eher $3 \mathrm{CaO} \cdot 2 \mathrm{SiO}_{2}$ sein als $4 \mathrm{CaO} \cdot 3 \mathrm{SiO}_{2}$.

Die Verbindung $3 \mathrm{CaO} \cdot 2 \mathrm{SiO}_{2}$ bildet mit $\alpha-\mathrm{CaO} \cdot \mathrm{SiO}_{2}$ ein eutektisches Gemisch von der Zusammensetzung $\mathrm{CaO}=54.5 \%, \mathrm{SiO}_{2}=$ $45.5 \%$ (Gewichtsprozente); dieses schmilat bei $1455^{\circ} \pm 2^{0}$. Sie bildet kein Eutektikum mit $2 \mathrm{CaO} \cdot \mathrm{SiO}_{2}$; der Quadrupelpunkt, bei dem diese beiden Verbindungen stabil nebeneinander und neben Flüssigkeit und Dampf bestehen, hat die Zusammensetzung $\mathrm{CaO}=$ $55.5 \%, \mathrm{SiO}_{2}=44.5 \%$; die entsprechende Temperatur ist $1475^{\circ} \pm 5^{\circ}$.

(3.) Calcium-ortho-silicat, $2 \mathrm{CaO} \cdot \mathrm{SiO}_{2} \quad(\mathrm{CaO}=65.0 \%$, $\mathrm{SiO}_{2}=35.0 \%$ ). Diese Verbindung tritt in drei Formen $\alpha, \beta, \gamma$ und wahrscheinlich in einer vierten $\beta^{\prime}$ auf, die monotrop ist. Die bei hohen Temperaturen stabile $\alpha$-Form schmilzt bej $2130^{\circ} \pm 20^{\circ}$; bei $1420^{\circ} \pm 2^{\circ}$ erfährt sie eine umkehrbare Umwandlung in die $\beta$-Form, die wiederum bei $675^{\circ}$ umkehrbar in die $\gamma$-Form übergeht. Die Umwandlung $\beta \rightarrow \gamma$ ist von einer Zunahme des Volumens um etwa $10 \%$ begleitet, wodurch die Masse in feinen Staub zersprengt wird. Diese Erscheinung, die man häufig beim Brennen von Portlandzement beobachtet, ist unter dem Namen „Verstäubung“ wohlbekannt.

$\alpha-2 \mathrm{CaO} \cdot \mathrm{SiO}_{2}$ kommt in unregelmäBigen Körnern vor, die so verworren und verzwillingt liegen, daß eine zufriedenstellende Messung ihrer optischen Eigenschaften außerordentlich schwierig ist. Sie zeigen gelegentlich prismatische Entwicklung mit guter Spalt- 
barkeit parallel zur Prismenachse. Zwillingsbildung ist eine charakteristische Eigenschaft; sie tritt häufig in äußerst komplizierter Form auf. Verschiedene Gruppen von Zwillingslamellen schneiden sich unter verschiedenen Winkeln und bilden auch einander durchdringende Massen, nicht unähnlich dem Mikroklin oder dem Leucit. Gelegentlich ist in einem dünnen Teilehen nur eine Gruppe polysynthetischer Zwillingslamellen vorhanden und es ist dann einem nach dem Albitgesetz verzwillingten Plagioklasschnitt ähnlich. Das Kristallsystem ist entweder monoklin oder triklin. Brechungsindizes $\alpha_{N a}=1.715$ $\pm 0.002, \quad \beta_{N a}=1.720 \pm 0.003, \quad \gamma_{N a}=1.737 \pm 0.002$. Doppelbrechung ziemlich stark, etwa 0.023; Winkel der optischen Achsen groß; Ebene der optischen Achsen: fast parallel mit der Längsrichtung der Kristalle; optischer Charakter: positiv. Die Auslöschungswinkel in verzwillingten Teilen wurden bisweilen mit so hohen Werten wie c : $\alpha^{\prime}=18^{\circ}$ beobachtet; allerdings traten kleinere Winkel häufiger auf. Die Härte liegt zwischen 5 und 6 .

$\beta-2 \mathrm{CaO} \cdot \mathrm{SiO}_{2}$ tritt in unregelmäßigen, abgerundeten Körnern, oftmals von prismatischer Gestalt, mit Spaltbarkeit parallel zur Prismenachse auf. Das Kristallsystem ist augenscheinlich rhombisch, wenngleich das Auftreten von Zwillingslamellen mit geringen Auslöschungswinkeln bis zu c: $\alpha^{\prime}=10^{\circ}$ in einigen der Körner, die der $\alpha$-Form angehören mögen, auf eine noch geringe Symmetrie deuten könnte.

Brechungsindizes: $\alpha_{N a}=1.717 \pm 0.002 ; \quad \gamma_{N a}=1.735 \pm 0.002 ;$ Doppelbrechung mittelgroß; die Interferenzfarben in den fein gepulverten Körnern gehen selten über das Blau der zweiten Ordnung hinaus; Winkel der optischen Achsen groß, optischer Charakter: positiv. Ebene der optischen Achsen parallel mit der Längenausdehnung. Die optischen Eigenschaften $\operatorname{der} \alpha$ - und der $\beta$-Form sind leider so ähnlich, daß es oft außerordentlich schwierig ist, zwischen beiden zu unterscheiden. Die verwickelte Zwillingsbildung der $\alpha$-Form scheint bei der $\beta$-Form nicht aufzutreten und ist augenscheinlich das beste Unterscheidungsmerkmal. Die Doppelbrechung der $\beta$-Form scheint auch etwas geringer zu sein als die der $a$-Form.

$\gamma-2 \mathrm{CaO} \cdot \mathrm{SiO}_{2}$ erhält man bei der Umwandlung der $\beta$-Form beim Abkühlen nur als feines Pulver durch die oben erwähnte „Verstäubung". Sie ist farblos, von prismatischer Tracht mit vollkommener Spaltbarkeit nach der Prismenachse. Gelegentlich fand man Andeutungen von Zwillingslamellen, parallel der Spaltrichtung, die kleine Auslöschungswinkel, nicht über $c: \gamma^{\prime}=3^{0}$ zeigten; ge- 
wöhnlich ist die Auslöschung parallel. Kristallsystem wahrscheinlich monoklin. Brechungsindizes: $\alpha_{N a}=1.642 \pm 0.002, \beta_{N a}=1.645 \pm$ $0.003, \gamma_{N a}=1.654 \pm 0.002$; Doppelbrechung mittelgro $B$, etwa 0.015 ; Winkel der optischen Achsen $2 \mathrm{E}$ ungefähr 520; Ebene der optischen Achsen normal zur Spaltrichtung; optischer Charakter: negativ. Diese Form des Orthosilicates ist leicht von der $\alpha$ - und $\beta$-Form durch ihre geringere Brechbarkeit zu unterscheiden, sowie durch die Lage ihrer Ebene der optischen Achsen und ihren optischen Charakter. $\beta^{\prime}-2 \mathrm{CaO} \cdot \mathrm{SiO}_{2}$ ist eine instabile Form, die man nur erhielt durch Abschrecken einer alten hydratisierten Probe von etwa 14250; sie konnte aus frischen Präparaten nicht erhalten werden, und wir haben sie in der Tat nur aus einem einzigen Muster gewinnen können und dann in feinen, gleichmäßigen Körnern, die augenscheinlich keine Spaltbarkeit zeigten. Der mittlere Brechungsindex ist etwa 1.715 und die Doppelbrechung sehr schwach. Interferenzfiguren sind schwierig zu erhalten wegen der Feinkörnigkeit und der schwachen Doppelbrechung; aber der optische Achsenwinkel scheint klein oder 0 zu sein, und der optische Charakter ist positiv. Die Form scheint monotrop zu sein, aber die bisher erlangten Beweise für die Existenz dieser Form sind durchaus noch nicht frei von allen Zweifeln.

4. Tricalciumsilicat $3 \mathrm{CaO} \cdot \mathrm{SiO}_{2} \quad\left(\mathrm{CaO}=73.6 \%, \quad \mathrm{SiO}_{2}=\right.$ $26.4 \%$ ) ist bei səinem Schmelzpunkt instabil, aber ungleich der Verbindung $3 \mathrm{CaO} \cdot 2 \mathrm{SiO}_{2}$ tritt es nicht als primäre Phase an irgendeinem Punkt der Flüssigkeitslinie des binären Systems Kalk-Kieselsäure auf. Wenngleich es eine bestimmte binäre Verbindung ist, so wurde es doch nur als primäre Phase in einem ternären System gefunden. Sie zerfällt bei $1900^{\circ} \pm 20^{\circ}$ in $\mathrm{CaO}$ und $2 \mathrm{CaO} \cdot \mathrm{SiO}_{2}$. Die Tatsache, daß diese Temperatur unterhalb der eutektischen Temperatur zwischen $\mathrm{CaO}$ und $2 \mathrm{CaO} \cdot \mathrm{SiO}_{2}$ (2065 ${ }^{\circ}$, Zusammensetzung $67,5 \% \mathrm{CaO}, 32.5 \% \mathrm{SiO}_{2}$ ) liegt, erklärt, warum $3 \mathrm{CaO} \cdot \mathrm{SjO}_{2}$ nicht in Berührung mit Schmelze im binären System vorkommt.

Diese Verbindung tritt in kleinen gleichmäßigen Körnern von nicht über $0.03 \mathrm{~mm}$ Durchmesser auf, ist farblos und augenscheinlich ohne Spaltbarkeit. Mittlerer Brechungsindex ungefähr 1.715; Doppelbrechung sehr schwach, nicht über 0.005. Die Körner erscheinen einachsig oder zweiachsig mit kleinem Winkel der optischen Achsen; optischer Charakter: negativ. Bei gewissen Präparaten aus ternären Gemischen wurden feine Zwillingslamellen mit niedrigen Auslöschungswinkeln beobachtet an einigen der Körner, woraus hervorging, daß das Kristallsystem möglicherweise monoklin ist. 
Es ist von Interesse, daß bei der Bildung dieser Verbindung aus den gemischten Oxyden bei $1400^{\circ}$ oder $1500^{\circ}$ zuerst Orthosilicat entsteht, das sich dann beim längeren Erhitzen mit freiem Calciumoxyd zu Tricalciumsilicat vereinigt.

\section{Das binäre System: Aluminiumoxyd-Silicium-2-oxyd.}

Das allgemeine Verhalten des Systemes AluminiumoxydSilicium-2-oxyd ergibt sich aus der Zeichnung von Fig. 2. In dieser

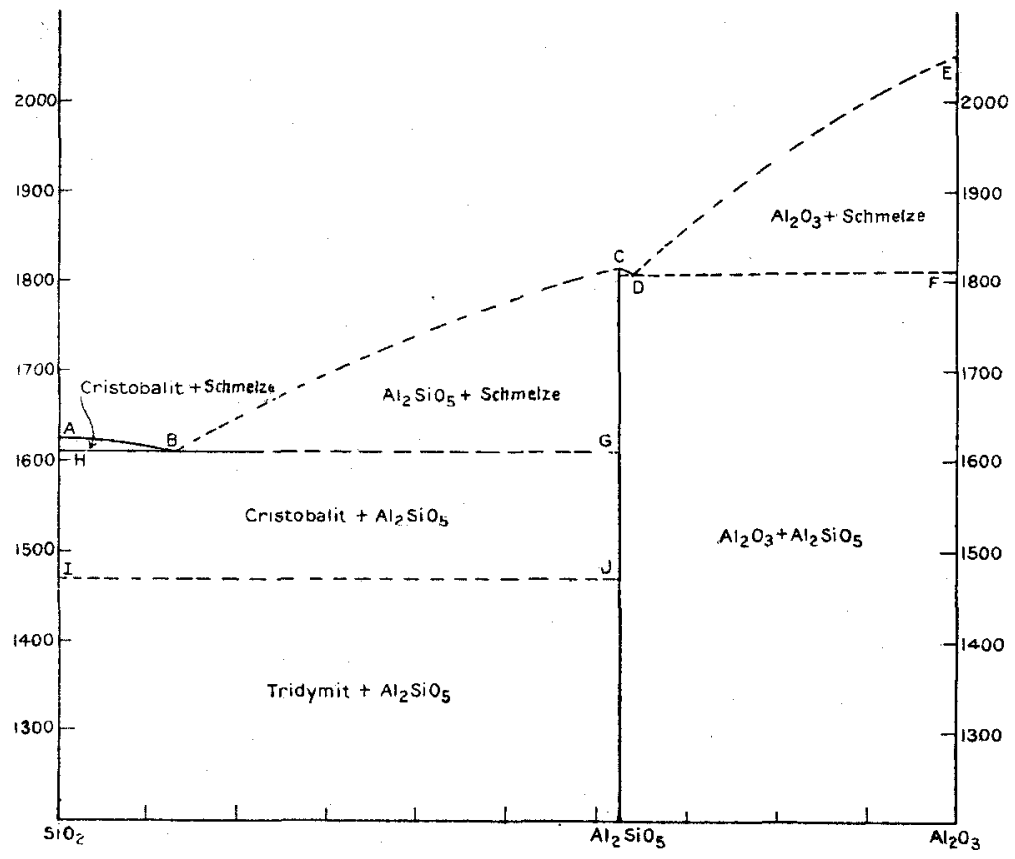

Fig. 2. Konzentrations -Temperatur-Diagramm des Systemes $\mathrm{Al}_{2} \mathrm{O}_{3}-\mathrm{SiO}_{2}$.

binären Reihe tritt nur eine Verbindung, der Sillimanit, $\mathrm{Al}_{2} \mathrm{O}_{3} \cdot \mathrm{SiO}_{2}$ auf; dieser schmilat bei $1816^{\circ} \pm 10^{\circ}$ und bildet eutektische Gemische mit Aluminiumoxyd sowohl wie mit Silicium-2-oxyd. Das Eutektikum zwischen Sillimanit $\mathrm{Al}_{2} \mathrm{O}_{3} . \mathrm{SiO}_{2}$ und $\mathrm{Al}_{2} \mathrm{O}_{3}$ hat die $\mathrm{Zu}$ sammensetzang $64 \% \mathrm{Al}_{2} \mathrm{O}_{3}, 36 \% \mathrm{SiO}_{2}$ und schmilzt bei $1810^{\circ} \pm 10^{\circ}$. Das Eutektikum zwischen Sillimanit und Cristobalit (die einzige Form von $\mathrm{SiO}_{2}$, die auf der Flüssigkeitslinie dieses Systemes erscheint) besteht aus $13 \% \mathrm{Al}_{2} \mathrm{O}_{3}$ und $87 \% \mathrm{SiO}_{2}$; es schmilzt bei $1610^{\circ}$. Zwei andere Formen von $\mathrm{Al}_{2} \mathrm{O}_{3} \cdot \mathrm{SiO}_{2}$, nämlich Andalusit und Cyanit oder Disthen, kommen in der Natur vor, sind aber bisher im Labo- 
ratorium nicht beobachtet worden; auch ist über ihre Beziehung zum Sillimanit nichts weiter bekannt geworden, als daß sie beim Erhitzen in diese Form übergehen. Die Erfahrungen im Laboratorium und im Felde, soweit sie vorhanden sind, zeigen, daß diese Formen in bezug auf Sillimanit monotrop sind.

Künstlicher Sillimanit kristallisiert aus der Schmelze in feinkörnigen, faserigen bis lattenförmigen Individuen, die, wie das natürliche Mineral, oft in dichten parallelen Gruppen auftreten. Endschnitte der Fasern haben rechteckigen Umriß, sind schwach doppelbrechend und löschen parallel den Diagonalen des Rechtecks aus. Im Mittelpunkt von fast jedem Schnitt ist ein kleiner Einschluß vorhanden, oft in Form eines Kreuzes, dessen Arme den Seiten parallel sind. Brechungsindizes $\alpha_{N a}=1.638 \pm 0.003, \beta_{N a}=1.642$ $\pm 0.003, \gamma_{N a}=1.653 \pm 0.003$; sie sind merklich niedriger als die des natürlichen Minerales; Doppelbrechung mittelgro $B, \gamma-\alpha$ etwa 0.014. Der Winkel der optischen Achsen ist nicht groß. Optischer Charakter: positiv; die spitze Bisektrix liegt in der Längsrichtung der Fasern, nach welcher deutlich Spaltbarkeit vorhanden ist. Kleine Einschlüsse einer weniger brechenden isotropen Substanz ( $n$ etwa 1.530) sind fast stets in den Präparaten von künstlichem Sillimanit vorhanden; sie bestehen augenscheinlich aus Glas. Eine zufriedenstellende Erklärung für ihr Auftreten ist nicht gefunden worden; es sei denn, daß Sillimanit bei seinem Schmelzpunkt instabil ist und in $\mathrm{Al}_{2} \mathrm{O}_{3}$ und Glas zerfällt. Dies würde auch die feinen, kreuzförmigen Einschlüsse, die oben erwähnt wurden, erklären. Die Temperatur des möglichen Zerfalles liegt jedoch so hoch (über $1800^{\circ}$ ), daß keine zufriedenstellenden Methoden zur Prüfung dieser Annahme vorhanden sind.

\section{Das binäre System: Calciumoxyd-Aluminiumoxyd.}

In diesem System treten vier verschiedene Verbindungen auf, nämlich 1. Tricalciumaluminat $\left(3 \mathrm{CaO} \cdot \mathrm{Al}_{2} \mathrm{O}_{3}\right), 2$. die Verbindung $5 \mathrm{CaO} .3 \mathrm{Al}_{2} \mathrm{O}_{3}$, 3. Calciumaluminat $\left(\mathrm{CaO} \cdot \mathrm{Al}_{2} \mathrm{O}_{3}\right)$ und 4. die Verbindung $3 \mathrm{CaO} .5 \mathrm{Al}_{2} \mathrm{O}_{3}$. Verschiedene dieser Verbindungen sind dimorph. Das Konzentrations-Temperatur-Diagramm ist in Fig. 3 dargestellt.

1. Tricalciumaluminat $3 \mathrm{CaO} . \mathrm{Al}_{2} \mathrm{O}_{3} \quad(62.2 \% \quad \mathrm{CaO}, \quad 37.8 \%$ $\mathrm{Al}_{2} \mathrm{O}_{3}$ ) ist bei seinem Schmelzpunkt nicht stabil, tritt aber als primäre Phase der Flüssigkeitslinie von Gemischen der Zusammensetzung zwischen 50 bis $59 \% \mathrm{CaO}$ und 50 bis $41 \% \quad \mathrm{Al}_{2} \mathrm{O}_{3}$ auf. Es 
zersetzt sich bei $1535^{\circ} \pm 5^{0}$ in $\mathrm{CaO}$ und Flüssigkeit; aus diesem Grund erhält man es am besten aus Glas seiner eigenen Zusammensetzung bei einer Temperatur unterhalb $1535^{\circ}$. Es bildet mit der Verbindung $5 \mathrm{CaO} .3 \mathrm{Al}_{2} \mathrm{O}_{3}$ ein eutektisches Gemisch der Zusammen-

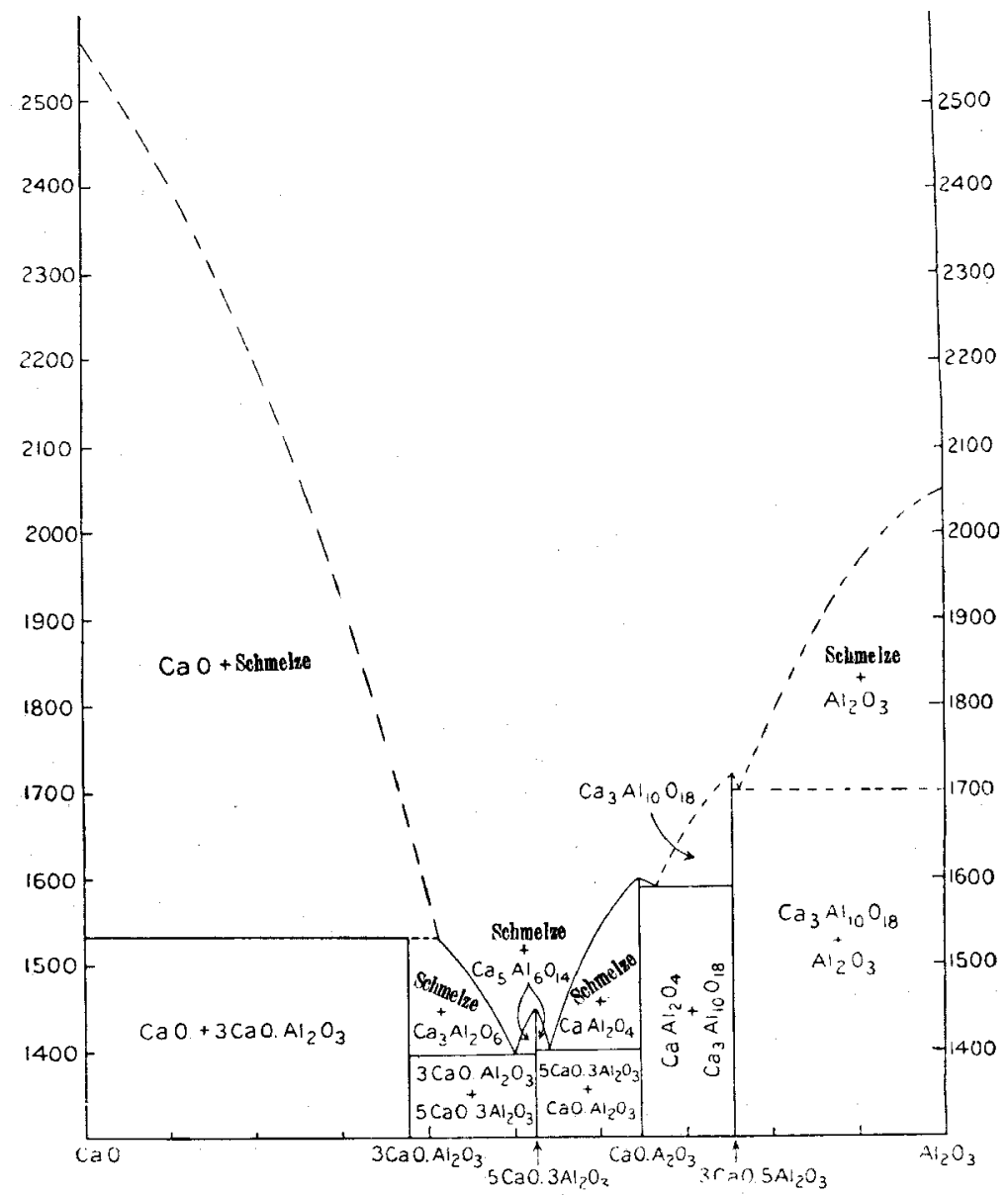

Fig. 3. Konzentrations -Temperatur-Diagramm des Systemes $\mathrm{CaO}-\mathrm{Al}_{2} \mathrm{O}_{3}$.

setzung $50 \% \mathrm{CaO}, 50 \% \mathrm{Al}_{2} \mathrm{O}_{3}$, das bei $1395^{\circ}$ schmilzt. Es bildet kein Eutektikum mit $\mathrm{CaO}$, aber das Gemisch $59 \% \mathrm{CaO}, 41 \% \mathrm{Al}_{2} \mathrm{O}_{3}$ ist der Quadrupelpunkt (invariant), bei dem diese beiden Verbindungen in Berührung mit Flüssigkeit und Dampf stabil sind; die Gleichgewichtstemperatur beträgt $1535^{\circ}$.

Diese Verbindung kommt in gleichmäßigen farblosen Körnern von $0.1 \mathrm{~mm}$ und geringerem Durchmesser vor, die oft hexagonalen 
oder rechteckigen Umriß haben, mit Andeutungen von unvollkommener Spaltbarkeit nach dem Oktaeder oder dem Rhombendodekaeder. Kristallsystem isometrisch. Brechungsindex $n_{N a}=$ $1.710 \pm 0.001$. Härte 6. Bruch muschelig. Glanz glasig. Gelegentlich wurden schwache, grave Interferenzfarben beobachtet, die augenscheinlich durch Spannungen hervorgerufen waren.

2. Die Verbindung $5 \mathrm{CaO} .3 \mathrm{Al}_{2} \mathrm{O}_{3}\left(47.8 \% \mathrm{CaO}, 52.2 \% \mathrm{Al}_{2} \mathrm{O}_{3}\right)$ kommt in zwei Formen vor, von denen I stabil ist, während II instabil und monotrop ist.

I. Die stabile Form schmilat bei $1455^{\circ} \pm 5^{0}$. Sie bildet mit der Verbindung $3 \mathrm{CaO} \cdot \mathrm{Al}_{2} \mathrm{O}_{3}$ das erwähnte eutektische Gemisch und mit Calciumaluminat ein eutektisches Gemisch der Zusammensetzung $47 \% \mathrm{CaO}, 53 \% \mathrm{Al}_{2} \mathrm{O}_{3}$, das bei $1400^{\circ} \pm 5^{0}$ schmilzt.

Die stabile Form kristallisiert in abgerundeten Körnern ohne bestimmte Kristallbegrenzung und bestimmte Spaltbarkeit. Glanz glasig; Bruch muschelig und oft unterbrochen; Härte 5. Kristallsystem isometrisch; Brechungsindex $n_{N a}=1.608 \pm 0.001$. Der Brechungsindex vom Glas derselben Zusammensetzung ist etwa 1.662.

II. Die instabile Form hat weder einen bestimmten Schmelzpunkt, noch ein Temperaturgebiet, in dem sie wirklich stabil ist. Man erhält sie nur unter besonderen Bedingungen beim Abkühlen, und gewöhnlich ist sie nicht gut in einzelnen Kristalliten entwickelt, sondern tritt in radialen Sphäruliten oder Aggregaten von Fasern auf, die sich überlappen und oft angenähert parallel sind. Kristalltracht faserig und prismatisch; Glanz glasig; Farbe gewöhnlich schwach grün; Härte etwa 5; Kristallsystem wahrscheinlich rhombisch. Brechungsindizes $\alpha_{N a}=1.687 \pm 0.002, \gamma_{N a}=1.692 \pm 0.002$; Doppelbrechung schwach; Winkel der optischen Achsen augenscheinlich groß; Ebene der optischen Achsen und der Auslöschung sind parallel mit der Faserrichtung; optischer Charakter augenscheinlich negativ, aber schwierig zu bestimmen wegen der einander überlappenden Fasern. Die tiefer gefärbten Körner sind pleochroitisch $\alpha=$ blaugrün, $\gamma=$ olivgrün; Absorption $\alpha>\gamma$.

3. Calciumaluminat $\mathrm{CaO} \cdot \mathrm{Al}_{2} \mathrm{O}_{3}\left(35.4 \% \mathrm{CaO}, 64.6 \% \quad \mathrm{Al}_{2} \mathrm{O}_{3}\right)$ schmilat bei $1600^{\circ} \pm 5^{0}$. Es bildet mit der Verbindung $5 \mathrm{CaO} .3 \mathrm{Al}_{2} \mathrm{O}_{3}$ das oben erwähnte eutektische Gemisch und mit der Verbindung $3 \mathrm{CaO} .5 \mathrm{Al}_{2} \mathrm{O}_{3}$ ein eutektisches Gemisch der Zusammensetzung 33.5 $\mathrm{CaO}, 66.5 \mathrm{Al}_{2} \mathrm{O}_{3}$, das bei $1590^{\circ} \pm 5^{0}$ schmilzt.

Es kristallisiert leicht in unregelmäßigen Körnern und oft in prismatischen bis faserigen Aggregaten. Zwillingsbildung ist eine 
charakteristische Eigensehaft, besonders hervortretend auf Schnitten fast normal zur spitzen Bisektrix. Derartige Schnitte sind in verwickelter Weise in ein hexagonales Maschenwerk von ineinander eingreifenden Sechsecken geteilt, die in verschiedenen Lagen auslöschen. Spaltbarkeit parallel der Faserrichtung ist gelegentlich angedeutet. Härte etwa 6.5; Glanz glasig. Kristallsystem monoklin oder triklin, wahrscheinlich monoklin. Brechungsindizes: $\alpha_{N a}=$ $1.643 \pm 0.002, \beta_{N a}=1.655 \pm 0.002, \gamma_{N a}=1.663 \pm 0.003 ;$ Doppelbrechung ziemlich stark, etwa 0.02. Winkel der optischen Achsen $2 V=36^{0} \pm 4^{0}$. Optischer Charakter : negativ.

4. Die Verbindung $3 \mathrm{CaO} .5 \mathrm{Al}_{2} \mathrm{O}_{3}\left(24.8 \% \mathrm{CaO}, 75.2 \% \mathrm{Al}_{2} \mathrm{O}_{3}\right)$ kristallisiert in zwei Formen:

I ist stabil, II ist instabil und augenscheinlich monotrop gegen I.

I. Diese Form schmilzt bei $1720^{\circ} \pm 10^{\circ}$. Sie bildet mit Calciumaluminat das oben erwähnte eutektische Gemisch und mit Aluminiumoxyd ein eutektisches Gemisch der Zusammensetzung $24 \% \mathrm{CaO}$, $76 \% \mathrm{Al}_{2} \mathrm{O}_{3}$, das bei $1700^{\circ} \pm 10^{\circ}$ schmilzt.

Sie kristallisiert leicht in abgerundeten farblosen Körnern, die oft Andeutung eines rechteckigen Umrisses zeigen. Spaltbarkeit schwach, wenn vorhanden. Die Härte beträgt etwa 6.5; Glanz ist glasig. Kristallsystem wahrscheinlich tetragonal. Brechungsindizes $\omega_{N a}=1.617 \pm 0.002, \varepsilon_{N a}=1.652 \pm 0.002$. Doppelbrechung stark, ungefähr 0.035; einachsig, optisch positiv. Andeutungen von zweiachsigem Charakter mit kleinem Winkel $2 E$ wurden gelegentlich beobachtet. Die Interferenzfiguren sind leicht zu erhalten, selbst an Körnern, die nur $0.01 \mathrm{~mm}$ Durchmesser haben. Die Körner enthalten oft feine, fadenartige Einschlüsse von höherem Brechungsvermögen, schwach doppelbrechend und isotrop, die für eine zufriedenstellende Bestimmung zu fein sind. Thre gesamte Menge ist sehr gering.

II. Die instabile Form kristallisiert gelegentlich aus der schnell abgekühlten Flüssigkeit. Sie kommt nur selten in den ternären Gemischen vor; hat keinen bestimmten Schmelzpunkt und augenscheinlich kein bestimmtes Gebiet wirklicher Stabilität. Sie wandelt sich bei hohen Temperaturen so schnell in die stabile Form um, daß völlig homogene Präparate nicht erhalten werden konnten. Kristalltracht prismatisch bis faserig mit mäßiger prismatischer Spaltbarkeit; Glanz glasig; Härte 5.5 bis 6 . Kristallsystem rhombisch oder monoklin, wahrscheinlich rhombisch, wenngleich bei 
einem Präparat Zwillingsbildung beobachtet wurde, die auf monokline Symmetrie deutet. Brechungsindizes: $\gamma_{N a}=1.674 \pm 0.002$, $\beta_{\text {Na }}=1.671 \pm 0.002$.

\section{Anwendung der Phasenregel auf ternäre Systeme.}

Es wird zweckmäBig sein, die allgemeinen Grundsätze, auf denen die experimentelle Untersuchung eines ternären Systemes beruht, auseinander $\mathrm{zu}$ setzen, bevor die angewandten Versuchsmethoden und die erhaltenen Ergebnisse mitgeteilt werden. Das wichtigste führende Prinzip ist die Phasenregel, die natürlich auf Gemische von mineralischen Oxyden, die nur bei hohen Temperaturen flüssig sind, ebenso anwendbar ist wie auf die gewöhnlichen Lösungen. In jedem Falle ist es notwendig, die Phasenregel in der geeigneten Weise anzuwenden; in der Tat ist dies das einzige Verfahren, derartig komplizierte Fragen, wie die Untersuchung des Systemes Calciumoxyd-Aluminiumoxyd-Silicium-2-oxyd, in Angriff zu nehmen; denn in diesem System haben wir nicht weniger als 14 verschiedene Verbindungen aufgefunden, die alle ein stabiles Existenzgebiet unter bestimmten Bedingungen besitzen.

\section{Anwendung der Phasenregel auf die Untersuchung der Gleichgewichte im System Calciumoxyd-Aluminiumoxyd-Silicium-2-oxyd.}

Für Systeme, die aus Calciumoxyd, Aluminiumoxyd und Silicium-2-oxyd aufgebaut sind, lassen sich gewisse Einschränkungen vornehmen, die die Bestimmtheit der Gleichgewichtsbedingungen nicht merklich beeinflussen, dagegen die Zahl der zu betrachtenden Möglichkeiten erheblich herabsetzen.

Da die Änderung des Dampfdruckes bei diesen drei Oxyden in dem zu untersuchenden Temperaturgebiet unwichtig ist, so können wir annehmen, daß unter konstantem Druck (Atmosphärendruck) jedes System durch die Freiheitsgrade, Temperatur und Konzentration definiert ist. Die Dampfphase muß jedoch jederzeit als vorhanden betrachtet werden. Als Flüssigkeiten sind diese 3 Oxyde in allen Verhältnissen mischbar, so daß für alle Systeme nur eine flüssige Faser auftritt. Aus der experimentellen Untersuchung wissen wir, daß die verschiedenen Oxyde and ihre Verbindungen als feste Stoffe praktisch nicht mischbar sind, so daß bei der Besprechung der theoretischen Möglichkeit die feste Lösung fortgelassen werden kann. 
Die Systeme, welche wir zu betrachten haben, sind demnach solche, in denen 1. der Dampfdruck des festen Stoffes und der Flüssigkeit während der Temperaturänderung wesentlich konstant bleibt, 2. nur eine flüssige Phase vorhanden ist und 3. feste Lösungen nicht gebildet werden.

Nach der Phasenregel ist $P+F=C+2$; da demnach in diesem Falle $C=3$, so ist die Anzahl der Phasen und Freiheitsgrade $\left(P+F^{\prime}\right)=5$, so $\operatorname{da} \beta F=0,1$ oder 2 sein kann.

Damit ein System aus drei Komponenten invariant sei, sind 5 Phasen notwendig; es sind daher zwei Systeme möglich: $S-S-S-S-V$ und $S-S-S-L-V$ ( $S=$ fest, $L=$ flüssig, $V=$ Dampf). Wenn das System monovariant sein soll, sind 4 Phasen notwendig. Es gibt zwei derartige mögliche Systeme: $S-S-S-V$ und $S-S-L-V$. Für ein divariantes System sind 3 Phasen erforderlich, und die folgenden zwei Systeme sind möglich: $S-S-V$ und $S-L-V$. Die festen Phasen können die Komponenten, binäre oder ternäre Verbindungen sein.

Aus den allgemeinen Gleichgewichtsbedingungen, die sich unter den erwähnten Verhältnissen aus der Phasenregel für Systeme aus drei Komponenten ableiten lassen, kann jetzt einiges über die Natur dieser Systeme gefolgert werden.

Die Gleichgewichtsbeziehungen eines Systemes aus drei Komponenten werden am leichtesten verstanden, wenn man die Zusammensetzung in ein gleichseitiges Dreieck einträgt; derselbe Maßstab wird für die binären Systeme auf den Seiten des Dreiecks wie für das ternäre System im Innern angewendet. In einer solchen Zeichnung ${ }^{1}$ werden die reinen Komponenten durch die Ecken des Dreiecks dargestellt, die invarianten Systeme durch Punkte (Quintupelpunkte), die monovarianten Systeme durch Linien (Grenzkurven) und die divarianten Systeme durch Felder, die vom Dreieck umschlossen werden. Alle Zusammensetzungen werden hier nach Gewichtsprozenten der Komponenten angegeben werden.

Die Einflüsse der Änderung der Variabeln (Temperatur) werden anschaulicher, wenn man ein räumliches Modell herstellt, indem man in jedem Punkt der Zeichenebene des Dreiecks senkrechte Linien errichtet, deren Länge proportional der Gleichgewichts-

${ }_{1}$ Roozeboom, Zeitschr. phys. Chem. 15 (1894), 13. - BANCRoft, Journ. phys. Chem. 1 (1897), 403. 
temperatur bei dieser Zusammensetzung ist. ${ }^{1}$ Für den vorliegenden Zweck jedoch werden Projektionen der ternären Kurven auf die Ebene des Dreiecks benutzt werden.

„Ein Theorem von Van RiJn Van ALkemade dient als sehr verläßlicher Führer für die Temperaturänderungen im Innern des Dreiecks. Wenn die beiden Punkte im Dreieck, die der Zusammensetzung zweier fester Phasen entsprechen, durch eine Linie verbunden werden, so wird die Temperatur, bei der diese zwei Phasen mit Lösung und Dampf im Gleichgewicht sind, steigen, wenn die Grenzkurve sich dieser Linie nähert und wird ein Maximum erreichen beim Schnitt, wenngleich die Grenzkurye vielfach nicht mehr stabil ist, bevor dieser Punkt erreicht wird. Wenn die beiden festen Phasen zwei der Komponenten sind, so ist die Linie, die ihre Zusammensetzungen verbindet, eine der Seiten des Dreiecks. Es ist demnach klar, daß die Temperatur immer steigen muß, wenn

1 Eine geistreiche Methode für die Herstellung eines räumlichen Konzentration-Temperaturmodelles ist von Herrn ENGLAND in diesem Laboratorium vorgeschlagen worden. Dieses Modell stellt genau die Konzentrationen und die entsprechenden Temperaturen für ein System aus drei Komponenten dar. Auf ein flaches Stück von gut abgelagertem Holz wird ein Stück Weißblech gut befestigt; dies dient als Basis für das feste Modell. Auf die Oberfläche des Bleches ist ein gleichseitiges Dreieck gezeichnet, in dem die Zusammensetzungen der binären und ternären Verbindungen, die Quadrupel- und Quintupelpunkte, sowie die Grenzkurven, die die Grenzen der verschiedenen ternären Felder bilden, als Linien aufgetragen sind. Das Konzentrations-Temperaturdiagramm für jedes binäre System (nicht allein die binären Systeme, von denen die Seiten des Dreiecks die Zusammensetzung geben, sondern auch die binären Systeme, die im ternären System eingeschlossen sind) und jede Grenzkurve wird aus einem Stück Weißblech ausgeschnitten. Die niedrigste Temperatur für jedes Konzentrations-Temperaturdiagramm muß natürlich eine gemeinsame Temperatur sein, nämlich die durch die Ebene der Basis des Modelles dargestellte. Jedes der Konzentrations-Temperaturdiagramme wird entlang seiner zugehörigen Konzentrationslinie auf der dreieckigen Basis senkrecht zur Ebene dieser Basis aufgestellt und dann auf diesem Platz festgelötet. Die entstehende Figur ist eine Skelettform, die die Zusammensetzungen und entsprechende Temperaturen der Verbindungen, die Quadrupelpunkte, Quintupelpunkte und Grenzkurven darstellt. Wenn wir nun in jeden Raum des Skelettes Gips einfüllen, dessen Oberfläche so gestaltet wird, daß sie mit dem Verlauf der jeden Raum um. gebenden Kurven übereinstimmt, so haben wir ein räumliches Modell, dessen Oberflächenpunkte die Zusammensetzungen und die entsprechenden Schmelztemperaturen der ternären Mischungen angeben. Umwandlungen, die in festen, ternären kristallisierten Gemischen auftreten, können natürlich in diesem Modell nicht zur Darstellung kommen. 
sie entlang einer Grenzkurve auf die Seite des Dreiecks vorrückt, wenn das Theorem von VAN ALKemade richtig ist."1

Die Nützlichkeit des Theorems von VAN Aukmmade wird deutlicher zutage treten, wenn wir es auf die Untersuchung der verschiedenen Typen der Dreikomponentensysteme anwenden.

\section{Typische Dreikomponentensysteme.}

Sechs einfache Typen von Systemen aus drei Komponenten ohne feste Lösungen sind in den Zeichnungen der Fig. 4 dargestellt, um einige der allgemeinen Prinzipien deutlich zu machen.
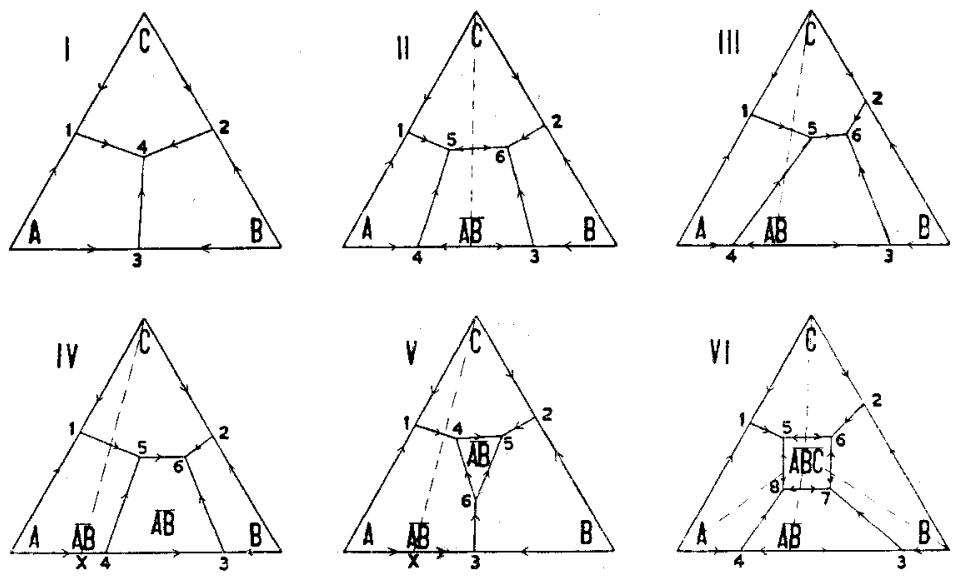

Fig. 4. Sechs typische Fälle von Systemen aus drei Komponenten.

Der einfachste Fall (1) ist der, bei dem die drei Komponenten die einzigen festen Phasen sind. $A, B, C$ sind die drei Komponenten, deren entsprechende Schmelzpunkte durch die Ecken des Dreiecks dargestellt werden, dessen Seiten die Schmelztemperaturen für die binären Gemische $A-B, B-C$ und $C-A$ darstellen. Die Pfeile auf den Seiten des Dreiecks zeigen die Richtung fallender Temperatur an und die Punkte 1, 2 und 3 sind die Quadrupelpunkte, in denen jedes Paar der Komponenten $C-A, B-C$ und $A-B$ im Gleichgewicht mit Lösung und Dampf existieren kann. Wenn, wie im vorliegenden Fall, die Quadrupelpunkte die niedrigsten Schmelz-

1 Bancroft, ,The Phase Rule", S. 149. 
temperaturen für jedes Zweikomponentensystem sind, bezeichnet man sie als eutektische Punkte.

Im Innern des Dreiecks ist für jede Komponente ein bestimmtes Gebiet (Feld), in dem es mit Lösung und Dampf im Gleichgewicht steht; mit anderen Worten, es gibt ein bestimmtes Feld, das die Schmelztemperaturen einer Komponente in Gemischen, die die anderen beiden Komponenten enthalten, darstellt. Die Linion 1-4, 2-4 und 3-4, die diese Felder trennen, sind die Grenzkurven, von denen jede die Bedingungen des 4-Phasen-Gleichgewichtes zwischen zwei Komponenten, Flüssigkeit und Dampf darstellt; das heißt, eine Grenzkurve gibt die Schmelztemperaturen von Gemischen zweier Komponenten an, die wechselnde Mengen der dritten Komponente enthalten. Der Ausgangspunkt einer Grenzkurve $1-4$ ist ein Quadrupelpunkt (1) in einem binären System $A-C$; wenn wir der Grenzkurve entlang in das ternäre System vorrücken, wird die entsprechende Gleichgewichtstemperatur fallen, wie das Theorem von Van Alkemade fordert. Die beiden Phasen in diesem Fall sind die Komponenten $A$ und $C$; nach dem Theorem müssen die Temperaturen entlang 4-1 steigen, wenn diese Linie sich $A-C$ nähert (die Temperaturen auf $\mathbf{1 - 4}$ müssen fallen, da diese Linie $A-C$ verläßt und in das ternäre System eintritt). Der Punkt 4, in dem sich die drei Grenzkurven begegnen, ist ein Quintupelpunkt, bei $\operatorname{dem} A, B, C$ im Gleichgewicht mit Lösung und Dampf sind. Da 4 die niedrigste Schmelatemperatur für irgendein Gemisch von $A, B$ und $C$ ist, bezeichnet man ihn als eutektischen Punkt.

Die nächst einfachen Fälle von Dreikomponentensystemen sind diejenigen, in denen eine binäre Verbindung auftritt. Sie werden veranschaulicht durch die Figuren II, III, IV und V (Fig. 4).

Bei II vereinigen sich $A$ und $B$ zur Verbindung $A B$, die bei ihrem Schmelzpunkt stabil ist. Das binäre System $-B$ wird dann ein Maximum (den Schmelzpunkt von $A B$ ) und zwei eutektische Punkte 3 und 4 besitzen. Im Dreieck werden dann 4 Felder sein; eines für jede der Komponenten $A, B, C$ und eines für die Verbinàung $A B$; ferner treten 5 Grenzkurven auf, von denen $5-6$ die einzige ist, die wir besonders betrachten müssen, da die anderen alle den bereits besprochenen analog sind. Die Grenzkurve 5-6, die gänzlich innerhalb des Dreiecks liegt, begrenzt die Felder der Komponente $C$ und der Verbindung $A B$. Wenn nun die Linie $C-A B 5-6$ kreuzt, so folgt aus dem Theorem von VaN Aukgmade, daß der Schnittpunkt die Maximaltemperatur der Grenze 5-6 sein 
wird. Die Punkte 5 und 6 werden demnach eutektische Punkte sein, wie durch die Pfeile angedeutet wird, die die Richtung der fallenden Temperatur auf den Grenzkurven angeben. Wenn jedoch, wie bei III, die Linie $C-A B$ die Grenzkurve 5-6 nicht kreuzt, so besitzt diese kein Maximum und die Temperatur auf dieser Linie fällt kontinuierlich von 5 nach 6 , und 6 ist ein eutektischer Punkt. Dies ist ein Fall, wo die Grenzkurve ein Maximum erreicht, aber bevor dies der Fall ist, hört die Verbindung auf, stabil zu sein. Punkt 5 ist ein Quintupelpunkt, aber kein eutektischer Punkt; d. h. 5 ist nicht die niedrigste Schmelztemperatur der drei Grenzkurven, die sich in ihm schneiden.

In IV ist $A B$ eine bei ihrem Schmelzpunkt instabile Verbindung, die bei der Temperatur des Punktes IV in festes $A$ und Flüssigkeit zerfällt, so daß die Zusammensetzung von $A B$ (Punkt $\mathrm{X}$ ) außerhalb des Feldes $4-5-6-3$ liegt, in dem $A B$ als primäre Phase auftritt. Punkt 4 ist ein Quadrupelpunkt, in $\operatorname{dem} A$ und $A B$ im Gleichgewicht mit Flüssigkeit und Dampf bestehen, aber 4 ist kein eutektischer Punkt. Im ternären System ist 6 ein eutektischer und 5 ein Quintupelpunkt, aber nicht eutektisch, wie im Fall III.

In $\mathrm{V}$ ist $A B$ eine instabile binäre Verbindung, die in festes $A$ und festes $B$ zerfällt, so daB $A B$ nicht auf der Flüssigkeitslinie binärer Gemische vorkommt. In ternären Gemischen jedoch ist $A B$ in Berührung mit Lösung stabil, weil die Schmelztemperaturen gewisser ternärer Gemische unterhalb der Zerfallstemperatur von $A B$ liegen. Das Feld für $A B$ ist von Kurven begrenzt, die vollständig innerhalb des Dreiecks liegen, und die Temperatur auf zweien dieser Kurven wird bis zu einem gemeinsamen Scheitel steigen, der auf das binäre System weist, in $\operatorname{dem} A B$ vorkommt.

Der Fall VI (Fig. 4) stellt ein System dar, in dem eine binäre und eine ternäre Verbindung $(A B$ und $A B C)$ auftreten, die beide einen bestimmten Schmelzpunkt haben. Das Feld für die ternäre Verbindung ist von Grenzkurven umgeben, die vollständig innerhalb des Dreiecks liegen; Zusammensetzung und entsprechender Schmelzpunkt der ternären Verbindung $A B C$ sind dargestellt durch das Maximum innerhalb des Feldes.

Wenn eine ternäre Verbindung bei ihrem Schmelzpunkt instabil ist, so kann sie entweder in zwei feste Phasen und Flüssigkeit zerfallen, in welchem Falle die Zusammensetzung außerhalb ihres Existenzgebietes liegt; oder aber sie kann in drei feste Stoffe dis- 
soziieren, und dann ist kein Feld vorhanden, in dem sie in Gleichgewicht mit Lösung vorkommt.

In einem ternären System kann natürlich eine beliebige Zahl binärer und ternärer, stabiler und instabiler Verbindungen vorkommen; aber die allgemeinen Regeln, die für die einfachen Fälle gelten, lassen sich auch auf die komplizierten Systeme anwenden. Aus diesem Grunde haben wir nicht alle Fälle, sondern nur einige typische Beispiele aufgenommen. Eine vollständigere Besprechung der in Dreikomponentensystemen auftretenden Möglichkeiten findet man in W. D. Bancrofts ,Phasenregel" oder in Roozebooms "Heterogene Gleichgewichte" III, 1.

Die Phasenregel und das Theorem von Van Alkemade ermöglichen uns auf diese Weise vorauszusagen, wieviel feste Phasen wir im Gleichgewicht unter bestimmten Bedingungen zu erwarten haben; und sie belehren uns nicht nur über die Lage der Maxima auf den Grenzkurven, sondern auch über die Richtung, nach welcher auf diesen Grenzkurven die Temperatur steigt und fällt; dies alles unterstützt die Einreihung neuer Verbindungen und die Bestimmung der Bedingungen, unter denen diese Verbindungen existieren. Die tatsächliche Feststellung der Verbindungen, die Bedingungen ihrer Existenz und ihre Beziehungen zu einander (sowie die Festlegung der Quintupelpunkte und Grenzkurven) kann jedoch nur mit Hilfe des Versuches geschehen.

\section{Experimentelle Methoden.}

Bei einer Untersuchung dieser Art sind drei allgemeine Versuchsmethoden vorhanden, die alle in weitem Maße hier Verwendung gefunden haben, wenngleich die dritte unserem Zweck am besten gedient hat.

1. Das erste Verfahren besteht darin, daß man die Energieänderungen, die im System auftreten und die durch ein Thermoelement registriert werden, feststellt und sich bemüht, in dieser Weise die Beziehungen der Phasen zu entwirren. Dies Verfahren ist ohne Wert, aber wie alle bei der Untersuchung neuer Gebiete benutzte Methoden gewinnt es an Kraft, wenn man es in Verbindung mit anderen Methoden benutzt, selbst wenn diese in bezug auf ihre praktische Anwendbarkeit von geringerer Brauchbarkeit sind. Bei Silikaten, wie bei Legierungen ist es viel leichter, die thermischen Daten zu deuten, wenn man die Natur der reagierenden Phasen kennt. 
2. Das zweite Verfahren besteht darin, daß man die verschiedenen Präparate lange Zeit bei geeigneten Temperaturen hält, damit die Reaktionen vollständig verlaufen und die Kristalle auf meßbare Größe wachsen können, wodurch die mikroskopische Prüfung sehr erleichtert wird.

3. Die dritte Methode hat den Zweek, die Grenzen der verschiedenen Felder, in denen besondere Phasen ihr stabiles Existenzgebiet besitzen, festzustellen, d. h. sie soll die Grenzkurven festlegen. In ternären Systemen bei gewöhnlicher Temperatur geschieht dies im allgemeinen so, daß man die zu untersuchende Phase zusetzt und feststellt, ob sie sich in der gesättigten Lösung auflöst oder nicht, oder indem man die gesättigte Lösung in geringem Umfang kristallisieren läßt, so daß die ersten sich bildenden Kristalle identifiziert werden können. ${ }^{1}$ Um dies Verfahren auch bei hohen Temperaturen anzuwenden, wird eine kleine Menge des Materiales der gewünschten Zusammensetzung in einen Ofen gebracht und bei konstanter Temperatur gehalten, bis beim Abschrecken (plötzliche Abkühlung durch Einwerfen in ein Bad aus Quecksilber oder Wasser), nur eine Art von Kristallen vorhanden ist und der Rest der Lösung glasig erstarrt, bevor er Zeit hat zu kristallisieren. Die genane Temperatur für ein gegebenes Gemisch muß durch eine Reihe von Versuchen unter ausreichender Abänderung der Versuchsbedingung bestimmt werden, um die durch Reaktionsträgheit oder Verzögerung der Kristallisation bedingten Wirkungen auszuschalten. Auf diese Weise findet man Temperaturen, bei denen die Beschickung völlig aus Glas besteht, und andere Temperaturen, bei denen das durch Abschrecken erhaltene Produkt völlig kristallisiert ist. Das Verfahren gibt auch ausreichend Zeit, daß die Kristalle zu meßbarer Größe anwachsen können, und unterstützt so die mikroskopische Prüfung. Mit anderen Worten, das Verfahren liefert die primäre Phase (Bodenkörper) eingebettet in das Glas (Lösung) und gestattet eine schnelle und sehr zufriedenstellende Aufzeichnung der Stabilitätsgebiete für jede Phase. Das Verfahren ist jedoch nicht ausführbar, wenn die Temperaturen höher sind, als die Anwendung eines Platinofens gestattet $\left(1600^{\circ}\right)$; der Iridium-Ofen $\left(1600^{\circ}\right.$ bis $\left.2100^{\circ}\right)$ ist hier nicht gut anwenabar. Natürlich arbeitet das Verfahren nicht so gut, wenn zwei Arten von Kristallen vorhanden sind. Um recht zufriedenstellende Ergebnisse, d. h. hinreichend große Kristalle der

1 Vgl. Roozkвoом, ,Heterogene Gleichgewichte“" 
primären Phase zu erhalten, soll die Beschickung auf einer Temperatur gehalten werden, die gerade unter der Temperatur vollständigen Schmelzens des fraglichen Gemisches liegt.

Das nach einem der drei Verfahren zu untersuchende Material wird folgendermaßen hergestellt:

Die feingepulverten Oxyde: Calciumoxyd (als Calciumkarbonat), Aluminiumoxyd, Silicium-2-oxyd, werden in den gewünschten Mengen gemischt, in einen Platintiegel gebracht und in einem Gasofen nach Fuetcher geschmolzen. Dann entfernt man die Beschickung aus dem Tiegel, zerkleinert sie in einem Stahlmörser, reibt sie in einem mechanisch betriebenen Achatmörser fein und schmilzt nochmals. Der Schmelzprozeß sowie das Feinreiben werden wenigstens dreimal wiederholt, um ein chemisch homogenes Produkt zu erhalten. Bei diesem Vorgehen sind zwei Operationen vorhanden, bei denen die Gefahr besteht, daß ein Teil der Beschickung verloren geht und fremdes Material hinzutritt:

1. beim Zerkleinern der Mischung im Stahlmörser und

2. beim Feinreiben im Achatmörser.

Wenn man jedoch sehr sorgfältig arbeitet, so können Mischungen auf diese Weise hergestellt werden, ohne da $B$ mehr als $0.2 \%$ fremdes Material hineinkommt oder daß sich der ursprüngliche Prozentgehalt der Oxyde um mehr als $0.2 \%$ ändert. Wir haben gefunden, daß Gemische von $20 \mathrm{~g}$ leicht zu handhaben sind und gewöhnlich für die optische und thermische Untersuchung ausreichen.

Die Mengen des Gemisches, die für jedes der drei Verfahren erforderlich sind, sind folgende:

1. Bei der Bestimmung der Energieänderungen fanden wir, daß zur Erzielung des schärfsten Knickes (der die Angabe der Energieänderung auf der Erhitzungskurve darstellt) eine Beschickung von 2.5 bis $5 \mathrm{~g}$ angewendet werden soll.

2. Für die Dauererhitzung zur Erzielung großer Kristalle wechselt die Beschickung von 1 bis $100 \mathrm{~g}$.

3. Bei der Abschreckung ist eine Beschickung von 0.1 bis $0.5 \mathrm{~g}$ für die mikroskopische Prüfung ausreichend, und diese Menge läßt sich leicht auf konstanter Temperatur halten.

Alle Versuche führte man in einer oxydierenden Atrosphäre (Luft) aus. Es kamen Öfen aus Platin oder Iridium zur Anwendung. Der Platinofen besteht aus einer Spule von Platindraht (Draht von $1.2 \mathrm{~mm}$ Durchmesser, Spule $2^{1 / 2}$ Zoll Durchmesser, 8 Zoll lang, 6 Windungen auf den Zoll), die auf der Innenseite eines Zylinders 
von unreinem Magnesit aufgewunden wurde, welcher mit Magnesia und einem Schamottezylinder isoliert wurde. Die Temperaturen im Platinofen werden mit einem Platin-Rhodium-Thermoelement durch Potentiometer und Galvanometer gemessen. ${ }^{1}$ Der Ofen wird erhitzt durch einen Strom aus einer Akkumulatorenbatterie (110 Volt, 300 Amp.-Stunden Kapazität) und die Temperatur im Ofen kann bei $1550^{\circ} 12$ Stunden lang bis auf $\pm 2^{\circ}$ konstant gehalten werden; bei niedrigeren Temperaturen läßt sich die Temperatur viel längere Zeit konstant halten. Der Iridiumofen besteht aus einem geraden Iridiumrohr von $18 \mathrm{~cm}$ Länge und $4 \mathrm{~cm}$ Durchmesser, welches durch einen Wechselstrom von niedriger Spannung geheizt wird. Die Temperaturen dieses Ofens mißt man mit einem optischen Pyrometer. ${ }^{2}$ Er wird nur für die Bestimmung von Stoffen gebraucht, die vollständig bei einer bestimmten Temperatur zwischen $1600^{\circ}$ und $2100^{\circ}$ schmelzen.

\section{Die experimentelle Untersuchung.}

\section{Allgemeine Beziehungen der binären Verbindungen im ternären System.}

Die Untersuchung der drei binären Systeme hat gezeigt, daß im ternären System neben den Komponenten selbst die folgenden binären Verbindungen auftreten: $\mathrm{CaO} . \mathrm{SiO}_{2} ; 3 \mathrm{CaO} .2 \mathrm{SiO}_{2} ; 2 \mathrm{CaO} . \mathrm{SiO}_{2}$; $3 \mathrm{CaO} \cdot \mathrm{Al}_{2} \mathrm{O}_{3} ; 5 \mathrm{CaO} .3 \mathrm{Al}_{2} \mathrm{O}_{3} ; \mathrm{CaO} \cdot \mathrm{Al}_{2} \mathrm{O}_{3} ; 3 \mathrm{CaO} .5 \mathrm{Al}_{2} \mathrm{O}_{3} ; \mathrm{Al}_{2} \mathrm{O}_{3} \cdot \mathrm{SiO}_{2}$; $3 \mathrm{CaO} . \mathrm{SiO}_{2}$.

In Fig. 5 sind die Zusammensetzungen der binären Verbindungen und die Quadrupelpunkte als Punkte auf den Seiten des ternären Diagrammes eingezeichnet. Diese Quadrupelpunkte bilden den Ausgang der Grenzkurven, die in das Dreieck von den Seiten eintreten; es kann aber nichts bestimmtes über den Verlauf dieser Grenzkurven aus den binären Systemen vorausgesagt werden, außer daß die Richtung fallender Temperatur von den Seiten des Dreiecks nach dem Innern liegen.

Für die Festlegung dieser Grenzkurven ist es jedoch von großem Wert, ihre Ausgangspunkte zu kennen; zu wissen, daB sie in gewissen Richtungen die Stabilitätsgebiete dieser Verbindungen begrenzen, die als primäre Phasen in den binären und ternären Systemen auftreten, und daß für jedes dieser Felder zwei solcher Grenzkurven

1 W. P. White, Potentiometeranordnung, insbesondere für hohe Temperaturen und thermoelektrische Arbeiten. Phys. Rev. 25 (1907), 334.

2 DAY, Shepherd, WRIGHT, Die Kalk-Kieselsäurereihe der Mineralien, Amer. Journ. Sci. (4) 22 (1906), 286. 
bestehen. Wenn diese beiden Grenzkurven von den beiden Seiten der Zusammensetzung einer Verbindung ausgehen, dann ist diese Verbindung bei ihrem Schmelzpunkt stabil, und ihre Zusammensetzung liegt in dem Feld, wo sie sich als primäre Phase ausscheidet. Solche stabilen binären Verbindungen sind die folgenden: $\mathrm{CaO} . \mathrm{SiO}_{2}$; $2 \mathrm{CaO} . \mathrm{SiO}_{2} ; 5 \mathrm{CaO} .3 \mathrm{Al}_{2} \mathrm{O}_{3} ; \mathrm{CaO} \cdot \mathrm{Al}_{2} \mathrm{O}_{3} ; 3 \mathrm{CaO} .5 \mathrm{Al}_{2} \mathrm{O}_{3} ; \mathrm{Al}_{2} \mathrm{O}_{3} \cdot \mathrm{SiO}_{2}$; die drei Komponenten verhalten sich in gleicher Weise. Wenn

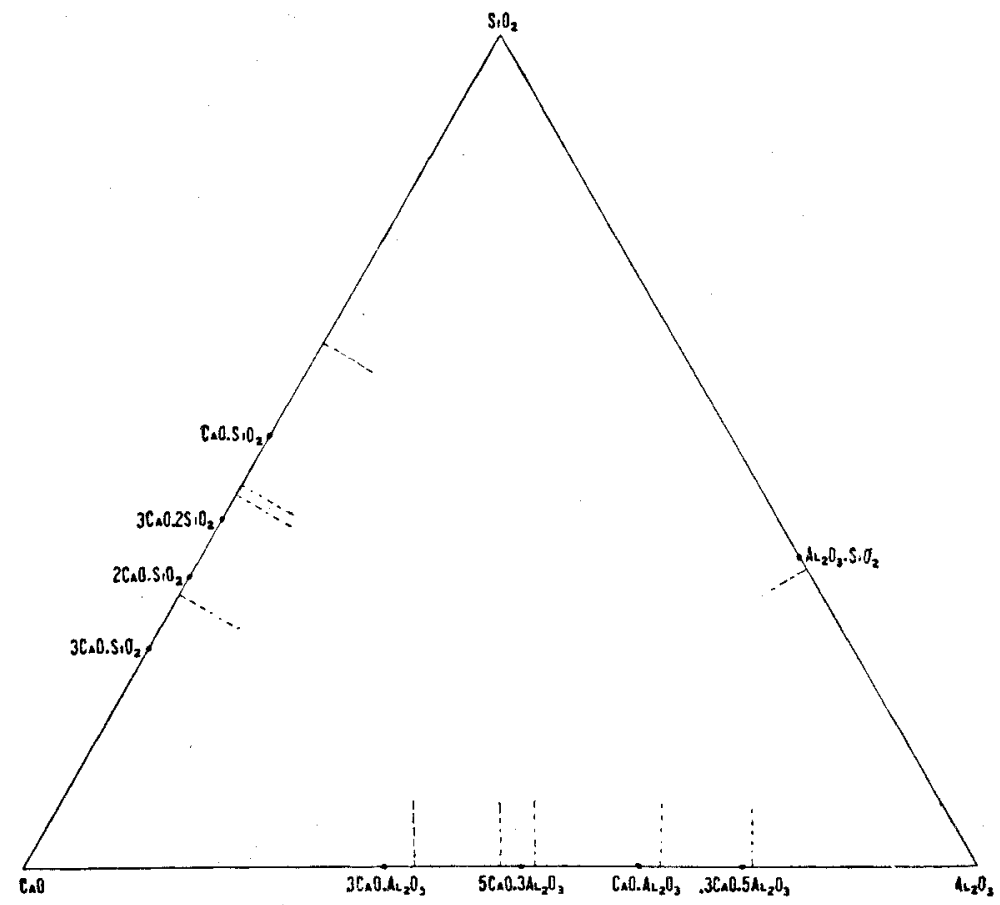

Fig. 5. Zusammensetzungen der binären Verbindungen und der Quadrupelpunkte, projiziert auf das ternäre Diagramm.

andererseits die Ausgangspunkte der beiden Grenzkurven auf derselben Seite der Zusammensetzung einer Verbindung liegen, dann ist diese bei ihrem Schmelzpunkt instabil, und ihre Zusammensetzung liegt außerhalb des ternären Feldes, in dem sie als primäre Phase auftritt. Derartige instabile binäre Verbindungen sind die folgenden: $3 \mathrm{CaO} \cdot \mathrm{Al}_{2} \mathrm{O}_{3}$ und $3 \mathrm{CaO} .2 \mathrm{SiO}_{2}$.

Für die instabile binäre Verbindung $3 \mathrm{CaO} . \mathrm{SiO}_{2}$, die nicht als primäre Phase in einem binären System auftritt, ist es nicht möglich, nach der Untersuchung der binären Systeme auszusagen, ob sie ein Stabilitätsgebiet im ternären System besitzt und von welcher 
Natur dieses sein mag; nur so viel läßt sich angeben: wenn das Feld drei Seiten besitzt, so muß die Temperatur auf zwei Grenzkurven bis zu einem gemeinsamen Gipfel steigen, der nach der Richtung des binären Systemes zeigt, in dem die Verbindung $3 \mathrm{CaO} . \mathrm{SiO}_{2}$ vorkommt.

Als wir die experimentelle Untersuchung des Systemes Calciumoxyd-Aluminiumoxyd-Silicium-2-oxyd aufnahmen, wußten wir schon beim Beginn, daß3 wenigstens 12 Verbindungen vorhanden wären, von denen mehrere in mehr als einer Kristallform auftreten, deren Stabilitätsbeziehungen im ternären System festgelegt werden mußten. Außer diesen 12 binären, konnte noch eine beliebige Anzahl ternärer Verbindungen vorhanden sein, die alle in dem untersuchten ternären System ihre Stabilitäts bedingungen zugewiesen erhalten mußten.

Der erste Schritt bei dieser Untersuchung bestand in der Herstellung von Gemischen, deren Zusammensetzung sich in Abständen von je $5 \%$ über das ganze Gebiet der ternären Gemische erstreckte. Dann bestimmte man die primäre Kristallphase für jedes dieser Gemische, welches bei Temperaturen unter $1600^{\circ}$ so abgeschreckt werden konnte, daß man eine einzige Kristallphase in Glas eingebettet erhalten konnte. Aus dieser vorläufigen Kenntnis erhielt man dann einen allgemeinen Begriff von der Anzahl und den Eigenschaften der Kristallphasen, die in Berührung mit Flüssigkeit stabil sind, sowie von ihren Wechselbeziehungen in den ternären Gemischen; mit anderen Worten, wir legten ungefähr die Felder mit ihren Grenzkurven und den Quintupelpunkten fest. Auf Grund dieser allgemeinen Daten wurden dann andere zwischenliegende Gemische hergestellt und ihre primären Phasen bestimmt. Dies sicherte eine genauere Festlegung der Grenzkurven und der Quintupelpunkte, so daß die Zusammensetzungen auf den Grenzkurven bis auf $10 \%$ und die der Quintupelpunkte bis auf einen Radius von $0.5 \%$ genau wurden.

Die Grenzen der Stabilitätsgebiete der verschiedenen Kristallphasen, die man in dieser Weise bestimmt hatte, sind in dem Dreieckdiagramm von Fig. 6 dargestellt. Die Punkte auf der Zeichnung stellen die untersuchten Zusammensetzungen dar; der Klarheit wegen sind sie in benachbarten Feldern verschieden bezeichnet. Diese Punkte jedoch umfassen nicht alle untersuchten Konzentrationen oder die vielen einzelnen Erhitzungsversuche und Prüfungen, die erforderlich waren, um die Beziehungen einer jeden Konzentration festzustellen. 
Für die vollständige Untersuchung des Systemes war die Darstellung ron etwa 1000 verschiedenen Gemischen notwendig, und mehr als 7000 Erhitzungsversuche und mikroskopische Prüfungen mußten ausgeführt werden.

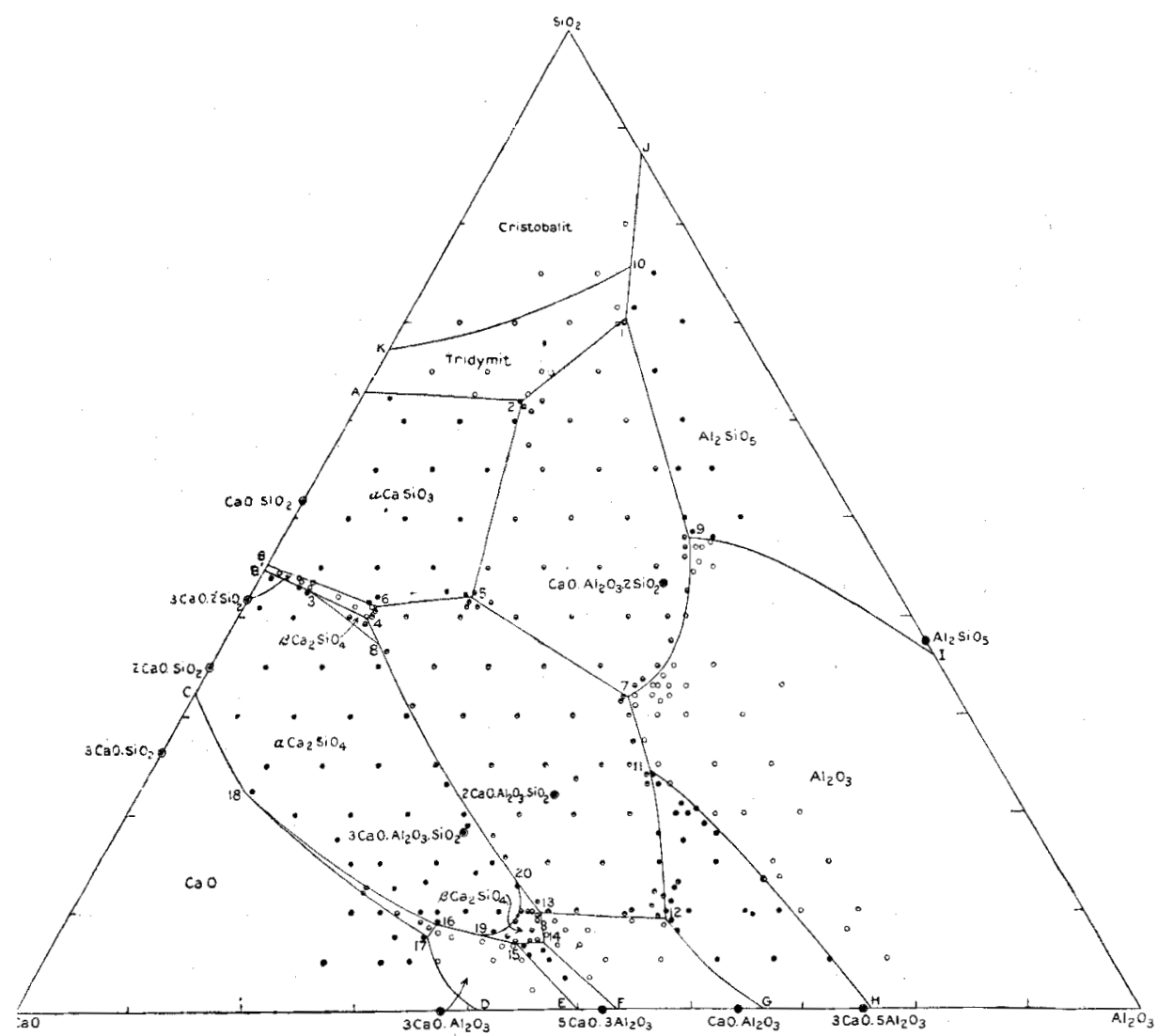

Fig. 6. Projektion des Konzentration-Temperatur-Diagrammes für das System $\mathrm{CaO}-\mathrm{Al}_{2} \mathrm{O}_{3}-\mathrm{SiO}_{2}$; enthält die Gemische, aus deren primären Phasen die Lage der Grenzkurven und Quintupelpunkte abgeleitet ist.

Wenn die Zusammensetzungen innerhalb eines jeden Feldes, entlang jeder Grenzkurve und bei jedem Quintupelpunkt bekannt sind, so ist es noch notwendig, die Zusammensetzung einer jeden unbekannten kristallisierten Phase, sowie die Gleichgewichtstemperaturen innerhalb der Felder auf den Grenzkurven und bei jedem Quintupelpunkt zu ermitteln. Die Prüfung von Fig. 6 zeigt, daß drei neue kristallisierte Phasen auftreten, die in den binären Reihen 
nicht vorkommen und die ternäre Verbindungen sind; es finden sich 14 Felder, 30 Grenzkurven und 21 Quintupelpunkte.

\section{Die ternären Verbindungen.}

Von den drei ternären Verbindungen erwiesen sich zwei als stabil in Berührung mit Flüssigkeit, und da das Feld für diese beiden Verbindungen je ein Maximum besitzt, wird ihre Zusammensetzung durch die Lage des Maximums dargestellt. Die Molekularzusammensetzung dieser beiden Verbindungen ist $\mathrm{CaO} \cdot \mathrm{Al}_{2} \mathrm{O}_{3} \cdot 2 \mathrm{SiO}_{2}$ und $2 \mathrm{CaO} \cdot \mathrm{Al}_{2} \mathrm{O}_{3} \cdot \mathrm{SiO}_{2}$.

Die dritte ternäre Verbindung erwies sich als instabil in Berührung mit Flüssigkeit, und daher war es notwendig, um ihre Zusammensetzung zu ermitteln, Gläser verschiedener Zusammensetzung zur Kristallisation zu bringen, bis man eine Zusammensetzung erhielt, in der die fragliche Verbindung die einzige vorhandene Phase war. Auf diese Weise ergab sich als Molekularzusammensetzung dieser Verbindung $3 \mathrm{CaO} \cdot \mathrm{Al}_{2} \mathrm{O}_{3} \cdot \mathrm{SiO}_{2}$.

Künstlicher Anorthit, $\quad \mathrm{CaO} . \mathrm{Al}_{2} \mathrm{O}_{3} \cdot 2 \mathrm{SiO}_{2} \quad(20.1 \% \quad \mathrm{CaO}$, $36.6 \% \quad \mathrm{Al}_{2} \mathrm{O}_{3}, 43.3 \% \mathrm{SiO}_{2}$ ). Reiner künstlicher Anorthit schmilzt bei $1550^{\circ} \pm 2^{\circ}$; sein ternäres Feld wird begrenzt von den ternären Feldern des Tridymits, von $a-\mathrm{CaO} . \mathrm{SiO}_{2}, 2 \mathrm{CaO} . \mathrm{Al}_{2} \mathrm{O}_{3} . \mathrm{SiO}_{2}, \mathrm{Al}_{2} \mathrm{O}_{3}$. $\mathrm{SiO}_{2}, \mathrm{Al}_{2} \mathrm{O}_{3}$; er bildet Quintupelpunktgemische mit Tridymit und $\alpha$-CaO. $\mathrm{SiO}_{2}$, mit Tridymit und $\mathrm{Al}_{2} \mathrm{O}_{3} . \mathrm{SiO}_{2}$, mit $\mathrm{Al}_{2} \mathrm{O}_{3} . \mathrm{SiO}_{2}$ and $\mathrm{Al}_{2} \mathrm{O}_{3}$, mit $\mathrm{Al}_{2} \mathrm{O}_{3}$ und $2 \mathrm{CaO} \cdot \mathrm{Al}_{2} \mathrm{O}_{3} \cdot \mathrm{SiO}_{2}$, sowie mit $2 \mathrm{CaO} \cdot \mathrm{Al}_{2} \mathrm{O}_{3} \cdot \mathrm{SiO}_{2}$ und $a-\mathrm{CaO} \cdot \mathrm{SiO}_{2}$.

In den Präparaten tritt er entweder in lattenförmigen Individuen, die nach der Kante (001) (010) verlängert sind, oder als tafelförmige Kristalle nach dem Seitenpinakoid $M(010)$ auf. Polysynthetische Zwillingsbildung nach dem Albitgesetz ist gewöhnlich und charakteristisch. Kristallsystem triklin. Brechungsindizes $\gamma_{N a}=1.589 \pm 0.001 ; \quad \beta_{N a}=1.585 \pm 0.001, \quad \alpha_{N a}=1.576 \pm 0.001$. (Diese Werte sind innerhalb der Fehlergrenzen mit denen des natürlichen Anorthits identisch.) Doppelbrechung nicht stark, $\gamma-a$ etwa 0.013. Auslöschungswinkel auf dem basalen Pinakoid ungefäh $r$ $35^{\circ}$; Winkel der optischen Achse groß; optischer Charakter negativ.

Die Verbindung $2 \mathrm{CaO} \cdot \mathrm{Al}_{2} \mathrm{O}_{3} \cdot \mathrm{SiO}_{2} \quad(40.8 \% \quad \mathrm{CaO}, \quad 37.2 \%$ $\mathrm{Al}_{2} \mathrm{O}_{3}, 22 \%, \mathrm{SiO}_{2}$ ) schmilzt bei $1590^{\circ} \pm 2^{0}$; ihr ternäres Feld ist begrenzt von den ternären Feldern für $\mathrm{CaO} . \mathrm{Al}_{2} \mathrm{O}_{3} .2 \mathrm{SiO}_{2}, \mathrm{Al}_{2} \mathrm{O}_{3}$, $3 \mathrm{CaO} .5 \mathrm{Al}_{2} \mathrm{O}_{3}, \mathrm{CaO} . \mathrm{Al}_{2} \mathrm{O}_{3}, \alpha-2 \mathrm{CaO} . \mathrm{SiO}_{2}, \beta-2 \mathrm{CaO} . \mathrm{SiO}_{2}, 3 \mathrm{CaO} .2 \mathrm{SiO}_{2}$, 
$\alpha$-CaO. $\mathrm{SiO}_{2}$; sie bildet Quintupelpunktsgemische mit $\mathrm{CaO} \cdot \mathrm{Al}_{2} \mathrm{O}_{3}$. $2 \mathrm{SiO}_{2}$ und $\mathrm{Al}_{2} \mathrm{O}_{3}$, mit $\mathrm{Al}_{2} \mathrm{O}_{3}$ und $3 \mathrm{CaO} .5 \mathrm{Al}_{2} \mathrm{O}_{3}$, mit $3 \mathrm{CaO} .5 \mathrm{Al}_{2} \mathrm{O}_{3}$ und $\mathrm{CaO} \cdot \mathrm{Al}_{2} \mathrm{O}_{3}$, mit $\mathrm{CaO} \cdot \mathrm{Al}_{2} \mathrm{O}_{3}$ und $\beta-2 \mathrm{CaO} . \mathrm{SiO}_{2}$ mit $\alpha-2 \mathrm{CaO} \cdot \mathrm{SiO}_{2}$ und $\beta-2 \mathrm{CaO} . \mathrm{SiO}_{2}$, mit $\beta-2 \mathrm{CaO} . \mathrm{SiO}_{2}$ und $3 \mathrm{CaO} .2 \mathrm{SiO}_{2}$, mit $3 \mathrm{CaO}$. $2 \mathrm{SiO}_{2}$ und $\alpha$-CaO. $\mathrm{SiO}_{2}$, mit $\alpha-\mathrm{CaO} . \mathrm{SiO}_{2}$ und $\mathrm{CaO} \cdot \mathrm{Al}_{2} \mathrm{O}_{3} .2 \mathrm{SiO}_{2}$.

Sie kristallisiert in klaren, glasartigen, farblosen Körnern, die deutliche aber nicht vollkommene basale Spaltbarkeit zeigen. Der Bruch ist muschelig, die Härte beträgt etwa 6. Spezifisches Gewicht 3.038. Kristallsystem tetragonal. Brechungsindizes $\omega_{N a}=1.669$ $\pm 0.001, \varepsilon_{N a}=1.658 \pm 0.001$; Doppelbrechung nicht stark, die Interferenzfarben sind gewöhnlich auf die der ersten Ordnung beschränkt; eine ausgesprochene Gelbfärbung ist bemerkenswert häufig. Einachsig; optisch negativ.

Die Verbindung $3 \mathrm{CaO} . \mathrm{Al}_{2} \mathrm{O}_{3} . \mathrm{SiO}_{2} \quad(50.9 \% \quad \mathrm{CaO}, \quad 30.9 \%$ $\mathrm{Al}_{2} \mathrm{O}_{3}, 18.2 \% \mathrm{SiO}_{2}$ ) ist bei ihrem Schmelzpunkt instabil, und im ternären System existiert kein Gebiet, in dem sie primäre Phase ist. Ihre Lage im ternären System ergibt sich aus Fig. 6. Sie zerfällt bei $1335^{\circ} \pm 5^{\circ}$ in $2 \mathrm{CaO} \cdot \mathrm{SiO}_{2}$ und $\mathrm{CaO} \cdot \mathrm{Al}_{2} \mathrm{O}_{3}$; demnach bildet sie sich am besten durch Kristallisation eines Glases ihrer Zusammensetzing bei einer Temperatur unterhalb 13350. Selbst dann erscheint das Präparat unter dem Mikroskop staubig und so wenig zufriedenstellend, daB es nicht mit Sicherheit festzustellen ist, ob es homogen ist. Die kristallisierte Masse ist gewöhnlich krypto- bis mikrokristallin und besteht aus sich überlappenden Fasern und Körnern. Die größeren Körner zeigen unter stärkeren Vergrößerungen die den Kristallskeletten eigenen Rippenstrukturen. Kristallsystem möglicherweise rhombisch. Brechungsindizes $\alpha_{N a}=1.675 \pm 0.005$, $\gamma_{N a}=1.685 \pm 0.005$. Doppelbrechung mittelgro $\beta, \gamma-\alpha$ etwa 0.01 . Winkel der optischen Achsen mittelgroß bis ziemlich groß. Optischer Charakter positiv. Die Ebene der optischen Achsen ist augenscheinlich parallel der Längsrichtung der Fasern.

\section{Die Stabilitäts-Gebiete.}

In dem vollständigen Diagramm Calciumoxyd-AluminiumoxydSilicium-2-oxyd ließen sich 14 getrennte Stabilitätsgebiete nachweisen; d. h. es sind 14 verschiedene Felder - je eins für jede im System vorkommende chemische Verbindung (einschlieBlich der ursprünglichen Komponenten) - , in denen eine einzige besondere Verbindung sich mit Flüssigkeit und Dampf im Gleichgewicht befindet. Jedes Feld kann betrachtet werden als Löslichkeitsgebiet 
einer bestimmten Verbindung, in Lösungen von zwei anderen bestimmten Verbindungen, oder als Schmelzgebiet einer Verbindung in Lösungen von drei bestimmten Verbindungen.

Die Schmelztemperaturen in jedem Feld erhielt man entweder aus Erhitzungskurven, die dazu dienten, die Temperatur, bei der die das Schmelzen begleitende Energieänderung eintritt, aufzuzeichnen (eine Schmelztemperatur in einem Feld ist der letzte auf der Erhitzungskurve auftretende Knick), oder durch Abschreckung, um die Temperatur zu bestimmen, oberhalb welcher ein Stoff als Glas (Flüssigkeit) und unterhalb welcher eine einzige kristallisierte Phase nach der optischen Prüfung mit dem Mikroskop in Glas eingebettet erhalten wird. Beide Methoden haben Verwendung gefunden, aber wenn die Energieänderungen wegen der Trägheit der Reaktion oder wegen Zusammentreffen mit anderen Energieänderungen nicht verzeichnet wird, dann ist die Abschreckungsmethode im allgemeinen die zuverlässigere. Wenn jedoch das Abschreckungsverfahren wegen der Schwierigkeit bei der Unterscheidung der festen Phasen nicht angewendet werden kann, dann ist die Aufnahme der Erhitzungskurve vorzuziehen. Für einige Punkte konnten beide Verfahren Anwendung finden; in diesen Fällen kontrollierten sich die beiden Versuchsergebnisse innerhalb der Fehlergrenżen $\left( \pm 5^{0}\right)$. Für gewisse Zusammensetzungen war es nicht möglich, die Schmelatemperaturen nach irgendeinem Verfahren zu ermitteln.

Wir werden jetzt die Felder getrennt betrachten; die Nummern und Buchstaben, die zu ihrer Bezeichnung dienen, entsprechen der Fig. 6. In den Tabellen sind nur Mittelwerte aus einer großen Anzahl von Bestimmungen mitgeteilt.

I. Das Feld von Silicium-2-oxyd $\left(\mathrm{SiO}_{2}\right)-K-A-2-1-J$. In dem Gebiet, wo $\mathrm{SiO}_{2}$ stabile primäre Phase ist, tritt es in den beiden Formen Tridymit und Cristobalit auf; $K-\mathbf{1 0}$ ist die Grenzkurve oberhalb derer Cristobalit und unterhalb derer Tridymit stabile Form ist. Wegen deł außerordentlichen Zähigkeit der Schmelzen in diesem Gebiet konnten zufriedenstellende Schmelztemperaturen weder durch Erhitzungskurven, noch durch Abschreckung erhalten werden; eine Vorstellung von dem allgemeinen Verlauf der Schmelzfläche in diesem Gebiet gewann man aus dem Verlauf der begrenzenden Kurven, nämlich der Gleichgewichtskurven $A-2 ; 2-1 ; 1-J$; $J-\mathrm{SiO}_{2} ; \mathrm{SiO}_{2}-A$.

II. Das Feld von $\mathrm{CaSiO}_{3}-(A-B-6-5-2)$. Wenngleich zwei 
Formen von $\mathrm{CaSiO}_{3}$ vorhanden sind, so tritt doch nur eine von ihnen im ternären System als primäre Phase auf, weil die Temperatur der eutektischen Punkte 2 und 5 höher liegt, als die Umwandlungstemperatur von $\beta$-in $\alpha$-Ca $\mathrm{SiO}_{3}$. Diese Umwandlungstemperatur ändert sich etwas mit der Zusammensetzung des ternären Gemisches, weil $\mathrm{CaSiO}_{3}$ eine kleine Menge - nicht über $2 \%$ - all der Verbindungen aufnehmen kann, mit denen es in den Grenzkurven zusammentrifft; diese Änderung zeigt die folgende Tabelle.

Tabelle 1.

Änderung der Umwandlungstemperatur $\alpha \rightleftharpoons \beta-\mathrm{CaSiO}_{3}$ durch Gegenwart anderer fester Phasen.

\begin{tabular}{|c|c|c|c|c|}
\hline \multicolumn{4}{|r|}{ Zusammensetzung } & \multirow{3}{*}{$\begin{array}{c}\text { Umwand- } \\
\text { lungs- } \\
\text { temperatur }\end{array}$} \\
\hline Gewi & ichtspro & zente & \multirow{2}{*}{ Gewichtsprozente } & \\
\hline $\mathrm{CaO}$ & $\mathrm{Al}_{2} \mathrm{O}_{3}$ & $\mathrm{SiO}_{2}$ & & \\
\hline $\begin{array}{l}48.2 \\
45 \\
50 \\
51.2 \\
46.3 \\
35 \\
48 \\
45\end{array}$ & $\begin{array}{c}0.9 \\
0.9 \\
10 \\
2 \\
5\end{array}$ & \begin{tabular}{l|}
51.8 \\
55 \\
50 \\
47.9 \\
52.8 \\
55 \\
50 \\
50
\end{tabular} & 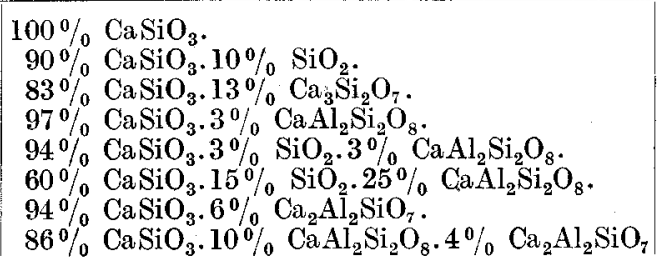 & $\begin{array}{l}1200^{\circ} \pm 2^{\circ} \\
1210^{\circ} \pm 5^{\circ} \\
1190^{0} \pm 5^{\circ} \\
1195^{0} \pm 5^{0} \\
1165^{0} \pm 5^{\circ} \\
1165^{0} \pm 5^{0} \\
1175^{0} \pm 5^{\circ} \\
1155^{0} \pm 5^{\circ}\end{array}$ \\
\hline
\end{tabular}

In dem Feld für $\alpha-\mathrm{CaSiO}_{3}$ lassen sich die Schmelztemperaturen sowohl aus den Erhitzungskurven wie aus Abschreckungsversuchen bestimmen; die mittleren Werte einer Anzahl Versuche mit jeder Zusammensetzung sind in Tabelle 2 gegeben.

\section{Tabelle 2.}

Schmelztemperatur verschiedener Gemische im Gebiet $A-B-6-5-2$. Primäre Phase ist $\alpha$-CaSiO 3 ( $\left.\mathrm{Smp} .1540^{\circ} \pm 2^{\circ}\right)$.

\begin{tabular}{c|r|r|r|r|r|r|r|r|r|r}
\hline \hline $\begin{array}{c}\text { Zusammensetzung } \\
\text { in } \\
\text { Gew. - \% }\end{array}$ & $\mathrm{CaO}_{2} \mathrm{Al}_{2} \mathrm{O}_{3}$ & $\mathbf{5 5}$ & 30 & $\mathbf{4 0}$ & $\mathbf{3 5}$ & $\mathbf{4 5}$ & 40 & 35 & 45 & 40 \\
$\mathrm{SiO}_{2}$ & 60 & 60 & $\mathbf{5 5}$ & $\mathbf{5 5}$ & $\mathbf{5 0}$ & $\mathbf{1 0}$ & $\mathbf{1 5}$ & $\mathbf{1 0}$ & $\mathbf{1 5}$ \\
\hline Schmelztemperatur & & 1408 & $\mathbf{1 3 2 2}$ & $\mathbf{1 4 4 9}$ & $\mathbf{1 3 7 4}$ & $\mathbf{1 4 8 1}$ & $\mathbf{1 4 1 6}$ & $\mathbf{1 3 3 0}$ & $\mathbf{1 4 0 2}$ & $\mathbf{1 3 4 5}$
\end{tabular}

III. Das Feld von $\mathrm{CaO} \cdot \mathrm{Al}_{2} \mathrm{O}_{3} \cdot 2 \mathrm{SiO}_{2}(1-2-5-7-9)$. Das Gebiet in dem $\mathrm{CaO} \cdot \mathrm{Al}_{2} \mathrm{O}_{3} \cdot 2 \mathrm{SiO}_{2}$ stabile primäre Phase ist, besitzt eine Maximaltemperatur von $1550^{\circ} \pm 2^{\circ}$ (Schmelzpunkt dieser Verbindung); die Schmelztemperaturen verschiedener Zusammensetzungen in diesem Feld sind in Tabelle 3 angegeben. 
Tabelle 3.

Schmelztemperatur verschiedener Gemische im Gebiet 1-2-5-7-9. Primäre Phase ist $\mathrm{CaO} \cdot \mathrm{Al}_{2} \mathrm{O}_{3} \cdot 2 \mathrm{SiO}_{2}$ (Smp. $\left.1550^{\circ} \pm 2^{0}\right)$.

\begin{tabular}{|c|c|c|c|c|c|c|c|c|c|c|c|c|c|}
\hline $\begin{array}{l}\text { Zusammen- } \\
\text { setzung in } \\
\text { Gew.- } \%\end{array}$ & $\mid$\begin{tabular}{c|}
$\mathrm{CaO}$ \\
$\mathrm{Al}_{2} \mathrm{O}_{3}$ \\
$\mathrm{SiO}_{2}$
\end{tabular} & $\begin{array}{l}20 \\
65\end{array}$ & $\begin{array}{l}18 \\
21 \\
61\end{array}$ & $\begin{array}{l}15 \\
25 \\
60\end{array}$ & $\begin{array}{l}25 \\
55\end{array}$ & $\begin{array}{l}25 \\
25 \\
50\end{array}$ & $\begin{array}{l}20 \\
30 \\
50\end{array}$ & $\begin{array}{l}30 \\
25 \\
45\end{array}$ & \begin{tabular}{l|}
25 \\
30 \\
45
\end{tabular} & $\begin{array}{l}20 \\
35 \\
45\end{array}$ & $\begin{array}{l}30 \\
30 \\
40\end{array}$ & $\begin{array}{l}25 \\
35 \\
40\end{array}$ & $\begin{array}{l}30 \\
35 \\
35\end{array}$ \\
\hline $\begin{array}{l}\text { Schm } \\
\text { tempe }\end{array}$ & & & & & & & & & & & & & \\
\hline
\end{tabular}

IV. Das Feld von $\mathrm{Al}_{2} \mathrm{SiO}_{5}(J-1-9-I)$. In diesem Gebiet erwies es sich als unausführbar, Schmelztemperaturen zu bestimmen, wegen der außerordentlichen Zähigkeit der Schmelzen in gewissen Teilen des Feldes und wegen sehr hochliegender Schmelztemperaturen in dem übrigen Teil. Trotzdem läBt sich aber der Verlauf der Schmelzfläche in diesem Gebiet ziemlich genau verfolgen, weil das Gebiet ziemlich eng ist und die Schmelztemperaturen an allen seinen Grenzen festgestellt sind.

V. Das Feld von $3 \mathrm{CaO} .2 \mathrm{SiO}_{2}\left(B^{\prime}-B-6-4\right)$. Dieses Feld unterscheidet sich in seiner Art etwas von dem vorhergehenden. Es ist nämlich diese Verbindung instabil, und ihre Zusammensetzung liegt demnach außerhalb des Feldes, in dem sie als primäre Phase auftritt. Der Verlauf dieses schmalen Feldes ergibt sich aus den Schmelztemperaturen seiner Grenzen, die bestimmt worden sind.

VI. Das Feld von 2 $\mathrm{CaO} . \mathrm{Al}_{2} \mathrm{O}_{3}$. $\mathrm{SiO}_{2}$ (6-5-7-11-12-13-4). Dieses Feld, dessen Grenzen völlig innerhalb des Dreiecks liegen, besitzt ein Maximum, welches den Schmelzpunkt für die Zusammensetzung der primären Phase $2 \mathrm{CaO} \cdot \mathrm{Al}_{2} \mathrm{O}_{3} \cdot \mathrm{SiO}_{2} \cdot 1590^{\circ} \pm 2^{\circ}$ darstellt. Die Schmelztemperaturen verschiedener Zusammensetzungen innerhalb dieses Feldes sind in Tabelle 4 zusammengestellt.

\section{Tabelle 4.}

Schmelztemperatur verschiedener Gemische im Gebiet 6-5-7-11-12-13-4. Primäre Phase ist 2CaO. $\mathrm{Al}_{2} \mathrm{O}_{3} \cdot \mathrm{SiO}_{2}\left(\mathrm{Smp} .1590^{\circ}\right)$.

\begin{tabular}{|c|c|c|c|c|c|c|c|c|c|c|}
\hline $\begin{array}{l}\text { Zusammensetzung } \\
\text { in } \\
\text { Gew.- } \%\end{array}$ & $\begin{array}{c}\mathrm{CaO} \\
\mathrm{Al}_{7} \mathrm{O}_{3} \\
\mathrm{SiO}_{8}\end{array}$ & $\begin{array}{l}40 \\
25 \\
35\end{array}$ & $\begin{array}{l}35 \\
30 \\
35\end{array}$ & $\begin{array}{l}45 \\
25 \\
30\end{array}$ & $\begin{array}{l}40 \\
30 \\
30\end{array}$ & $\begin{array}{l}\mathbf{3 5} \\
\mathbf{3 5} \\
30\end{array}$ & $\begin{array}{l}30 \\
40 \\
30\end{array}$ & \begin{tabular}{|l|}
31 \\
41.5 \\
27.5
\end{tabular} & $\begin{array}{l}45 \\
30 \\
25\end{array}$ & $\begin{array}{l}35 \\
40 \\
25\end{array}$ \\
\hline Schmelztemperatur & & 1475 & 1430 & 1554 & 1558 & 1503 & 1394 & 1450 & 1575 & 1580 \\
\hline $\begin{array}{l}\text { Zusammensetzung } \\
\text { in } \\
\text { Gew. } \%\end{array}$ & $\begin{array}{l}\mathrm{CaO} \\
\mathrm{Al}_{2} \mathrm{O}_{3} \\
\mathrm{SiO}_{3}\end{array}$ & \begin{tabular}{|l|}
46.3 \\
33.7 \\
20.0
\end{tabular} & $\begin{array}{l}45 \\
35 \\
20\end{array}$ & $\begin{array}{l}36 \\
46 \\
18\end{array}$ & \begin{tabular}{|l|}
48.8 \\
35.6 \\
15.6
\end{tabular} & $\begin{array}{l}45 \\
40 \\
15\end{array}$ & $\begin{array}{l}40 \\
45 \\
15\end{array}$ & \begin{tabular}{|}
47.6 \\
38.0 \\
14.4
\end{tabular} & $\begin{array}{l}47.0 \\
39.4 \\
13.6\end{array}$ & $\begin{array}{l}40 \\
50 \\
10\end{array}$ \\
\hline mperatur & & 1548 & & $=7$ & 1400 & $1 \mathrm{rac}$ & 50 & $7 \mathrm{ird}$ & & 51 \\
\hline
\end{tabular}


VII. Das Feld vom Aluminiumoxyd $\left(\mathrm{Al}_{2} \mathrm{O}_{3}\right)\left(7-\mathrm{I}_{-} \mathrm{Al}_{2} \mathrm{O}_{3}\right.$ $H-11)$. In dem vorläufigen Bericht war ein Feld angegeben, zwischen diesem Gebiet und dem für $\mathrm{Al}_{2} \mathrm{SiO}_{5}$, das einer Verbindung von unbekannter Zusammensetzung und unbekannten Eigenschaften angehören sollte. Die sorgfältigere Untersuchung hat gezeigt, daß die vermutete neue Verbindung nichts als Aluminiumoxyd ist. Die meisten Schmelztemperaturen in diesem Feld sind so hoch, daß sie sich der Bestimmung entziehen; für gewisse Zusammensetzungen sind sie in Tabelle 5 zusammengestellt.

Tabelle 5 .

Schmelztemperatur verschiedener Gemische im Gebiet 7-I- $\mathrm{Al}_{2} \mathrm{O}_{3}-H-11$. Primäre Phase ist $\mathrm{Al}_{2} \mathrm{O}_{3}$ (Smp. 2050 ). ${ }^{1}$

\begin{tabular}{c|c|l|l|l|l|l|l|l|l}
\hline \hline $\begin{array}{c}\text { Zusammensetzung } \\
\text { in } \\
\text { Gew.- } \%\end{array}$ & $\mathrm{CaO}_{0}$ & $\mathbf{1 4}$ & 18.5 & 20 & 23.5 & 28.5 & 29 & 30 & 29 \\
$\mathrm{Al}_{2} \mathrm{O}_{3}$ & 38.5 & 39 & 40 & 41.5 & 39.5 & 40 & 42.5 & 50.5 \\
$\mathrm{SiO}_{2}$ & 47.5 & 42.5 & 40 & 35 & 32 & 31 & 27.5 & 20.5 \\
\hline \hline Schmelztemperatur & & 1575 & 1550 & 1560 & 1530 & 1395 & 1430 & 1475 & 1550
\end{tabular}

VIII. Das Feld von Calcium-ortho-silikat $\left(\mathrm{Ca}_{2} \mathrm{SiO}_{4}\right)$ $\left(B^{\prime}-4-14-15-16-C\right) . \quad \mathrm{Ca}_{2} \mathrm{SiO}_{4}$ kommt in drei Formen vor, von denen zwei $\left(\alpha\right.$ und $\beta$ ) stabil sind, während eine $\left(\beta^{\prime}\right)$ instabil ist; alle drei finden sich in diesem Feld. Die $\beta$-Form ist stabil in den Gebieten 19-20-14-15 und 3-4-8, während nahe bei diesem letzten Gebiet die $\beta^{\prime}$-Form recht häufig auftritt. Im übrigen Teil des Feldes ist die $\alpha$-Form stabil.

Wenngleich $\mathrm{Ca}_{2} \mathrm{SiO}_{4}$ praktisch keine Änderung seiner optischen Eigenschaften in seinem ganzen Existenzgebiet zeigt, findet doch die Umwandlung $\beta \rightleftharpoons \alpha$ bei verschiedenen Temperaturen statt, die von der Zusammensetzung des untersuchten Gemisches abhängen, wie sich aus Tabelle 6 ergibt.

Tabelle 6.

Änderung der Umwandlungstemperatur $\beta \rightarrow \alpha \mathrm{Ca}_{2} \mathrm{SiO}_{4}$ durch Gegenwart einer weiteren festen Phase.

\begin{tabular}{c|c|c|c|c|c|c|c}
\hline \hline $\begin{array}{c}\text { Zusammensetzung } \\
\text { in } \\
\text { Gew.- } \%\end{array}$ & $\mathrm{CaO}_{0}$ & $\mathbf{6 5}$ & 60 & 60 & $\mathbf{5 5}$ & $\mathbf{5 2}$ & $\mathbf{5 8}$ \\
$\mathrm{Al}_{2} \mathrm{O}_{3}$ & - & 5 & 30 & 35 & 38 & 33 \\
$\mathrm{SiO}_{2}$ & $\mathbf{3 5}$ & $\mathbf{3 5}$ & 10 & 10 & 10 & $\mathbf{9}$ \\
\hline \hline Umwandlungstemperatur & & $\mathbf{1 4 2 0}$ & 1415 & 1395 & $\mathbf{1 3 9 5}$ & 1415 & $\mathbf{1 3 9 5}$
\end{tabular}

$\mathrm{Ca}_{2} \mathrm{SiO}_{4}$ nimmt keine merklichen Mengen von irgendeiner Verbindung, mit der es in diesem System zusammenkommt, in fester

${ }^{1}$ Kanolt, J., Wash. Acad. Sci. 3 (1913) 315. 
Lösung auf. Im größten Teil dieses Feldes sind die Schmelztemperaturen zu hoch, als daß sie genau bestimmt werden könnten. Die ermittelten Temperaturen sind in Tabelle 7 zusammengestellt.

Tabelle 7 .

Schmelztemperatur verschiedener Gemische im Gebiet

$B^{\prime}-4-14-15-16-C$. Primäre Phase ist $\mathrm{Ca}_{2} \mathrm{SiO}_{4}$ (Smp. $2130^{\circ} \pm 20^{\circ}$ ).

\begin{tabular}{c|c|c|c|c|c|c|c|c}
\hline \hline $\begin{array}{c}\text { Zusammensetzung } \\
\text { in }\end{array}$ & $\mathrm{CaO}^{\text {Gew. - } / 0}$ & 55 & 50 & 50 & 50 & 50.7 & 51.3 & 50.9 \\
$\mathrm{Al}_{2} \mathrm{O}_{3}$ & 5 & 10 & 15 & 17.5 & 22 & 29.7 & 30.9 \\
$\mathrm{SiO}_{3}$ & 40 & 40 & 35 & 32.5 & 27.3 & 19 & 18.2 \\
\hline \hline Schmelztemperatur & & 1625 & 1400 & 1500 & 1550 & 1625 & 1590 & 1570 \\
\hline Zusammensetzung & $\mathrm{CaO}$ & 50 & 57 & 60 & 52 & 50 & 49 & 50 \\
in & $\mathrm{Al}_{2} \mathrm{O}_{3}$ & 32.8 & 30 & 27.5 & 38 & 40 & 42 & 42 \\
Gew.- & $\mathrm{SiO}_{3}$ & 17.2 & 13 & 12.5 & 10 & 10 & 9 & 8 \\
\hline Schmelztemperatur & & 1535 & 1580 & 1600 & 1460 & 1420 & 1390 & 137.5
\end{tabular}

IX. Das Feld von $3 \mathrm{CaO} .5 \mathrm{Al}_{2} \mathrm{O}_{3}(11-12-G-H)$. $3 \mathrm{CaO} .5 \mathrm{Al}_{2} \mathrm{O}_{3}$ kommt in zwei Formen vor, einer stabilen und einer instabilen, die man gelegentlich in Schmelzen findet. Die Schmelztemperaturen der stabilen Form sind in Tabelle 8 zusammengestellt.

Tabelle 8.

Schmelztemperatur verschiedener Gemische im Gebiet

11-12-G-H. Primäre Phase ist $3 \mathrm{CaO} .5 \mathrm{Al}_{2} \mathrm{O}_{3}\left(\mathrm{Smp} .1720^{\circ} \pm 10^{\circ}\right)$.

\begin{tabular}{c|c|c|c|c|c|c}
\hline $\begin{array}{c}\text { Zusammensetzung } \\
\text { in } \\
\text { Gew.- } \%\end{array}$ & $\mathrm{ClO}_{2} \mathrm{Al}_{2}$ & 49 & 29 & 34.3 & 35 & 37 \\
$\mathrm{SiO}_{2}$ & 21 & $\mathbf{1 9}$ & $\mathbf{5 2 . 7}$ & 55 & $\mathbf{5 4}$ \\
\hline Schmelztemperatur & & 1540 & $\mathbf{1 5 9 0}$ & 1570 & $\mathbf{1 5 9 0}$ & $\mathbf{1 5 0 9}$ \\
\hline
\end{tabular}

X. Das Feld von $\mathrm{CaO} \cdot \mathrm{Al}_{2} \mathrm{O}_{3}$. Tabelle 9 enthält die Schmelztemperaturen von verschiedenen Zusammensetzungen aus diesem Feld.

Tabelle 9 .

Schmelztemperatur verschiedener Gemische im Gebiet F-G-12-13-14. Primäre Phase ist $\mathrm{CaO} . \mathrm{Al}_{2} \mathrm{O}_{3}$ (Smp. $\left.1600^{\circ} \pm 5^{0}\right)$.

\begin{tabular}{|c|c|c|c|c|c|c|c|c|}
\hline $\begin{array}{c}\text { Zusammensetzung } \\
\text { in } \\
\text { Gew.- } \%\end{array}$ & $\begin{array}{c}\mathrm{CaO} \\
\mathrm{Al}_{2} \mathrm{O}_{3} \\
\mathrm{SiO}_{2}\end{array}$ & $\begin{array}{r}45 \\
47 \\
8\end{array}$ & $\begin{array}{c}48.5 \\
45 \\
6.5\end{array}$ & $\begin{array}{r}36.7 \\
58.1 \\
5.2\end{array}$ & $\begin{array}{r}45 \\
50 \\
5\end{array}$ & $\begin{array}{r}40 \\
55 \\
5\end{array}$ & $\begin{array}{r}47 \\
49 \\
4 \\
\end{array}$ & $\begin{array}{r}47.5 \\
49.5 \\
3.0\end{array}$ \\
\hline Schmelztemperatur & & 1456 & 1361 & 1549 & 1468 & 1535 & 1432 & 1421 \\
\hline
\end{tabular}

XI. Das Feld von $5 \mathrm{CaO} .3 \mathrm{Al}_{2} \mathrm{O}_{3}(E-F-14-15)$. $5 \mathrm{CaO} .3 \mathrm{Al}_{2} \mathrm{O}_{3}$ tritt in einer stabilen und einer instabilen Form auf; diese erhält man aus Schmelzen recht häufig. Der Verlauf der Schmelzfläche auf dem engen Gebiet für die stabile Form ergibt sich aus den Schmelztemperaturen der Grenzen, die ermittelt wurden. 
XII. Das Feld von $3 \mathrm{CaO} \cdot \mathrm{Al}_{2} \mathrm{O}_{3}(E-15-16-17-D) .3 \mathrm{CaO} \cdot \mathrm{Al}_{2} \mathrm{O}_{3}$ ist eine bei ihrem Schmelzpunkt instabile Verbindung, deren $\mathrm{Zu}$ sammensetzung demnach außerhalb des Stabilitätsfeldes liegt. Tabelle 10 enthält die Schmelztemperaturen verschiedener Zusammensetzungen aus diesem Feld.

Tabelle 10.

Schmelztemperaturen verschiedener Gemische im Gebiet $E-15-16-17-D$. Primärphase ist $3 \mathrm{CaO} \cdot \mathrm{Al}_{2} \mathrm{O}_{3}$.

\begin{tabular}{c|c|c|c|c|c|c|c}
\hline Zusammensetzung & $\mathrm{CaO}$ & $\mathbf{5 8 . 5}$ & $\mathbf{5 7 . 5}$ & $\mathbf{5 2 . 5}$ & $\mathbf{6 0}$ & $\mathbf{5 5}$ & $\mathbf{5 2 . 5}$ \\
in & $\mathrm{Al}_{2} \mathrm{O}_{3}$ & 33.5 & $\mathbf{3 5}$ & 41 & $\mathbf{3 5}$ & $\mathbf{4 0}$ & 42.5 \\
Gew.-\% & $\mathrm{SiO}_{2}$ & 8 & $\mathbf{7 . 5}$ & $\mathbf{6 . 5}$ & $\mathbf{5}$ & $\mathbf{5}$ & $\mathbf{5}$ \\
\hline \hline Schmelztemperatur & & 1448 & 1435 & $\mathbf{1 3 7 0}$ & 1490 & $\mathbf{1 4 3 7}$ & 1390
\end{tabular}

XIII. Das Feld von Tricalciumsilikat, $3 \mathrm{CaO} . \mathrm{SiO}_{2}(16-$ 17-18). $3 \mathrm{CaO} . \mathrm{SiO}_{2}$ ist ebenso wie $3 \mathrm{CaO} \cdot \mathrm{Al}_{2} \mathrm{O}_{3}$ bei seinem Schmelzpunkt instabil, aber ungleich $3 \mathrm{CaO} \cdot \mathrm{Al}_{2} \mathrm{O}_{3}$, liegt das Stabilitätsfeld für $3 \mathrm{CaO} \cdot \mathrm{SiO}_{2}$ gänzlich innerhalb des ternären Systems. Dies Feld ist nur so schmal, daß der Verlauf der Schmelzfläche aus den Temperaturen der Grenzlinien abgeleitet wurde.

XIV. Das Feld von Calciumoxyd, CaO (D-CaO-C). Praktisch sind alle Schmelztemperaturen in diesem Feld zu hoch für die Bestimmung und demnach wurde der Verlauf der Schmelzfläche aus den Schmelztemperaturen der Grenzlinien abgeleitet.

\section{Die Grenzkurven.}

Eine Grenzkurve ist die Linie, die zwei Felder trennt und die Temperaturen und Konzentrationen darstellt, bei denen die feste Phase des einen Feldes neben der festen Phase des anderen Feldes, sowie neben Flüssigkeit und Dampf im Gleichgewicht ist.

Die Grenzlinien von Fig. 6 stellen die Projektion der Linien des räumlichen Konzentration-Temperatur-Modelles auf die horizontale Ebene dar. Um den Verlauf der Temperaturen auf diesen Linien zu veranschaulichen, haben wir Projektionen auf eine vertikale Ebene ausgeführt und erhalten so die Grenzkurven von Fig. 7, bei denen die Zahlen und Buchstaben denen von Fig. 6 entsprechen. Die Ordinaten stellen experimentell bestimmte Temperaturen dar.

Die Richtung steigender Temperatur entlang den Grenzkurven (Fig. 7) kann vorausgesagt werden durch Anwendung des Theorems von Van Alkemade auf die Projektionen dieser Kurven (Fig. 6). Betrachten wir z. B. die Grenze 5-2 in Fig. 6 zwischen $\alpha$-Ca $\mathrm{SiO}_{3}$ 


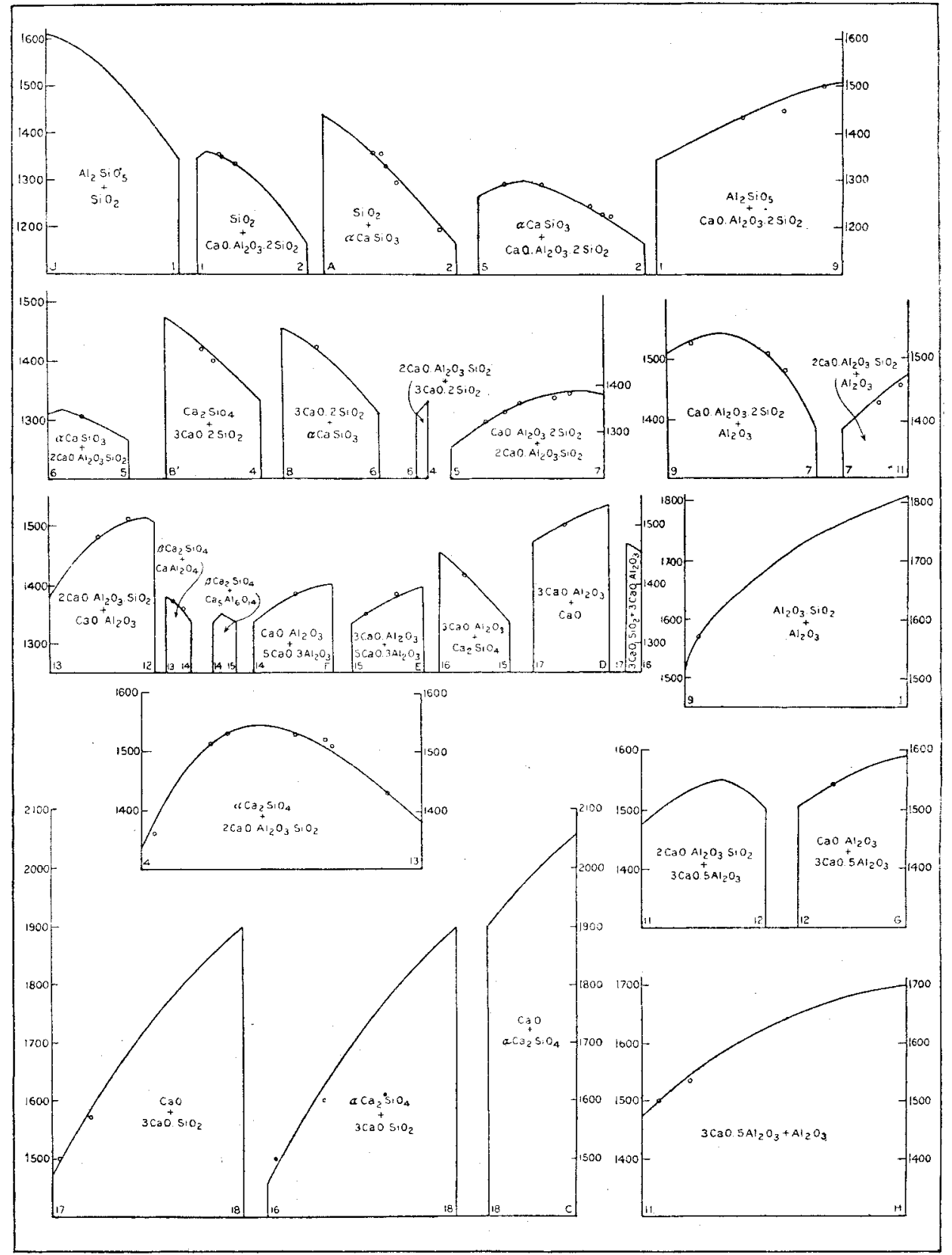

Fig. 7.

Temperatur-Konzentrations-Diagramme von 29 Grenzkurven, die die Grenzen der Felder im ternären System $\mathrm{CaO}-\mathrm{Al}_{2} \mathrm{O}_{3}-\mathrm{SiO}_{2}$ bestimmen. 
und $\mathrm{CaO} \cdot \mathrm{Al}_{2} \mathrm{O}_{3} \cdot 2 \mathrm{SiO}_{2}$. Es ist ganz augenscheinlich, daß eine gerade, die Zusammensetzungen der beiden Verbindungen verbindende Linie die Grenze 5-2 schneiden muß, und aus dem Theorem von Alkemade folgt dann, daß der Schnittpunkt ein Temperaturmaximum auf dieser Grenze 5-2 darstellt. Das Konzentrations-TemperaturDiagramm für die Grenze 5-2 in Fig. 7 zeigt dies Maximum. In Fig. 6 finden sich 9 Grenzlinien, auf denen man Maximaltemperaturen zu erwarten hätte; und das Konzentrations-TemperaturDiagramm (Fig. 7) zeigt, daß auf allen diesen Grenzkurven experimentell ein solches Maximum aufgefunden ist. Wenn wir nun die Grenze 1-9 zwischen $\mathrm{Al}_{2} \mathrm{SiO}_{5}$ und $\mathrm{CaO} \cdot \mathrm{Al}_{2} \mathrm{O}_{3} .2 \mathrm{SiO}_{2}$ betrachten, so ist augenscheinlich, daß eine gerade Linie zwischen den Zusammensetzungen dieser beiden Verbindungen die Grenze 1-9 nur dann schneiden würde, wenn man die Kurve über den Punkt 9 hinaus verlängerte. Demnach folgt aus dem Theorem von ALkmmade, daß die Temperaturen von 1-9 kontinuierlich von Punkt 1-9 ansteigen. Das Konzentrations-Temperatur-Diagramm für die Grenze 1-9 in Fig. 7 zeigt, daß dies der Fall ist. Es sind 19 von diesen Grenzkurven vorhanden, auf denen die Temperatur kontinuierlich steigt, d. h. 19 Grenzkurven zeigen kein Maximum.

Angenäherte Temperaturen auf den Grenzkurven können abgeleitet werden durch Extrapolation aus den Schmelztemperaturen, die für Punkte innerhalb der Felder bestimmt sind; aber für die genaueren Temperaturen sind Erhitzungskurven und Abschreckungsversuche erforderlich. Für die experimentelle Bestimmung der Temperatur eines Punktes auf der Grenzkurve ist es nicht notwendig, ein Gemisch der Zusammensetzung dieses Punktes anzuwenden, vielmehr kann jede Zusammensetzung benutzt werden, die auf einer geraden Linie liegt, welche durch den auf der Grenzkurve zu bestimmenden Punkt und die Zusammensetzung einer der beiden festen Phasen geht, die sich auf dieser Grenzkurve begegnen. Der Grund hierfür ergibt sich deutlich, wenn wir die aufeinanderfolgenden Änderungen betrachten, die beim Erhitzen eines ternären Gemisches stattfinden.

Auf Erhitzungskurven für ternäre Gemische entspricht der erste Knick (Wärmeabsorption) gewöhnlich der Temperatur eines Quintupelpunktes. Setzt man die Erhitzung fort, so geht das Schmelzen weiter, und das Gleichgewicht folgt einer Grenzkurve, bis die eine der beiden im Gleichgewicht mit Flüssigkeit und Dampf vorhandenen Phasen völlig geschmolzen ist; bei diesem Punkt finden wir den zweiten Knick, der die Schmelztemperatur des betreffenden 
Punktes auf der Grenzkurve darstellt. Jeder Punkt der Grenzkurven stellt die Zusammensetzung der Flüssigkeit dar, die bei diesem Punkt im Gleichgewicht ist mit dem noch festen Teil des Gemisches; aber wenn wir der Grenzkurve entlang gefolgt sind, bis sie die gerade Linie schneidet, die durch die Zusammensetzung des untersuchten ternären Gemisches und die Zusammensetzung der primären Phase des Gemisches geht, dann verläßt der Ort der Punkte, die die Zusammensetzung der flüssigen Phase darstellen, die Grenzkurve und verläuft in das benachbarte Feld auf der geraden Linie.

Eine vollständigere Besprechung der Änderungen, die beim Erhitzen eines ternären Gemisches stattfinden, wird bei der Betrachtung der ternären Kristallisationskurven erfolgen. Augenblicklich schien es ausreichend zu zeigen, daß die Temperatur für irgendeinen Punkt auf einer Grenzkurve bestimmt werden kann aus einer Anzahl verschiedener Gemische; daß ferner für irgendein ternäres Gemisch der Punkt, bei dem seine Erhitzungskurve die Grenzkurve verlassen wird, bekannt ist; und $\mathrm{da} B$ der zweite Knick auf einer Erhitzungskurve gewöhnlich die Temperatur eines Punktes auf der Grenzkurve ist. Für die ternären Gemische, bei denen der zweite Knick der Erhitzungskurve keine Temperatur der Grenzkurve ist, wird es notwendig, durch Abschrecken die Temperatur zu bestimmen, bei welcher während des Erhitzens das Gemisch die Zusammensetzung die Grenzkurve verläßt. Dies geschieht, indem man die Temperatur bestimmt, oberhalb welcher man nach Beobachtung mit dem Mikroskop eine feste kristallisierte Phase in Glas eingebettet erhält und unterhalb welcher zwei kristallisierte Phasen in Glas (Flüssigkeit) eingebettet sind.

Die für diese Temperaturen auf den Grenzkurven erhaltenen Daten werden nicht mitgeteilt werden, da die für diese Linien wesentlichen Temperaturen später als Quintupel- und Quadrupelpunkte angegeben werden.

Die Quintupelpunkte. Ein Quintupelpunkt, der gemeinsame Punkt für drei Grenzkurven, ist derjenige Punkt, bei dem drei feste Phasen neben Lösung und Dampf im Gleichgewicht sind. Ein Quintupelpunkt hat eutektischen Charakter, wenn er die niedrigste Temperatur der drei Grenzkurven darstellt, denen er gemeinsam angehört.

Die Temperatur eines Quintupelpunktes kann an irgendeinem Gemisch der bei diesem Quintupelpunkt vorhandenen festen Phasen bestimmt werden (da alle ternären Gemische mehr oder weniger von der Mischung des Quintupelpunktes enthalten) entweder durch 
Erhitzungskurven oder durch Abschreckversuche, durch die es ermöglicht wird, die Temperatur festzulegen, oberhalb welcher zwei kristallisierte Phasen in Glas eingebettet erhalten werden und unterhalb welcher drei feste kristallisierte Phasen entstehen; das heißt: die Temperatur, bei der in einem ternären Gemisch zuerst Glas entsteht, ist die Temperatur des Quintupelpunktes für dies Gemisch. Bei der Besprechung der Daten für die Quintupelpunkte werden wir zuerst die eutektischen Punkte betrachten, von denen 8 vorhanden sind, und später die 13 Quintupelpunkte ohne eutektischen Charakter.

Eutektische Punkte. Bei der Behandlung von Legierungen, die mehr oder weniger von einem eutektischen Gemisch enthalten, ist oft besprochen worden, was man unter ,eutektischer Struktur“" versteht; sie wird durch Benutzung des Mikroskopes leicht erkannt. Beim Arbeiten mit Silikatschmelzen sind Erscheinungen dieser Art nur selten zu beobachten; mit anderen Worten, ein eutektisches Gemisch von Silikaten hat im allgemeinen keine besondere Struktur, die sich von anderen Gemischen unterscheidet. Andererseits sind eutektische Gemische von Silikaten außerordentlich feinkörnig, und es ist sehr schwierig, Kristalle zu entwickeln oder wachsen zu lassen in einem Gemisch, das große Mengen des Eutektikums enthält.

Es folgt nunmehr eine Liste der eutektischen Gemische, die die Zusammensetzung nach Gewichtsprozenten von $\mathrm{CaO}, \mathrm{Al}_{2} \mathrm{O}_{3}$ und $\mathrm{SiO}_{2}$ sowie die entsprechenden Temperaturen enthält. Alle Daten, auf denen die Temperatur eines einzelnen eutektischen Punktes beruht, sind mitgeteilt; aus Mangel an Raum ist es jedoch nur möglich, einen Wert (das Mittel einer großen Zahl von Bestimmungen) für alle anderen eutektischen Punkte anzugeben. Die Nummer, welche jedem eutektischen Punkt beigefügt ist, entspricht der Nummer in Fig. 6.

Punkt 2 ist das Eutektikum für $\mathrm{SiO}_{2}, \alpha-\mathrm{CaSiO}_{3}$ und $\mathrm{CaO} \cdot \mathrm{Al}_{2} \mathrm{O}_{3}$. $2 \mathrm{SiO}_{2}\left(23.25 \% \mathrm{CaO}, 14.75 \%, \mathrm{Al}_{2} \mathrm{O}_{3}, 62 \%, \mathrm{SiO}_{2}\right)$. Seine Schmelztemperatur $\left(1165^{0} \pm 5^{0}\right)$ ist abgeleitet aus den in Tabelle $11 \mathrm{zu-}$ sammengestellten Werten für verschiedene Gemische.

Punkt 1 ist das Eutektikum für $\mathrm{Al}_{2} \mathrm{SiO}_{5}, \mathrm{CaO} . \mathrm{Al}_{2} \mathrm{O}_{3} .2 \mathrm{SiO}_{2}$ und $\mathrm{SiO}_{2}\left(9.8 \% \mathrm{CaO}, \mathbf{1 9 . 8 \%} \mathrm{Al}_{2} \mathrm{O}_{3}, 70.4 \% \mathrm{SiO}_{2}\right) ; 1345^{0} \pm 5^{0}$.

Punkt 5 ist das Eutektikum von $\mathrm{CaO} . \mathrm{Al}_{2} \mathrm{O}_{3} .2 \mathrm{SiO}_{2}, \mathrm{CaSiO}_{3}$ und $2 \mathrm{CaO} . \mathrm{Al}_{2} \mathrm{O}_{3} \cdot \mathrm{SiO}_{2}\left(38 \% \mathrm{CaO}, 20 \% \mathrm{Al}_{2} \mathrm{O}_{3}, 42 \% \mathrm{SiO}_{2}\right) ; 1265^{\circ} \pm 5^{0}$.

Punkt 6 ist das Eutektikum von $2 \mathrm{CaO} \cdot \mathrm{Al}_{2} \mathrm{O}_{3} \cdot \mathrm{SiO}_{2}, \mathrm{CaSiO}_{3}$ und $3 \mathrm{CaO} .2 \mathrm{SiO}_{2}\left(47.2 \% \mathrm{CaO}, 11.8 \% \mathrm{Al}_{2} \mathrm{O}_{3}, 41 \% \mathrm{SiO}_{2}\right) ; 1310^{\circ} \pm 5^{0}$.

Punkt 7 ist das Eutektikum für $\mathrm{Al}_{2} \mathrm{O}_{3}, 2 \mathrm{CaO} \cdot \mathrm{Al}_{2} \mathrm{O}_{3} \cdot \mathrm{SiO}_{2}$ und $\mathrm{CaO} . \mathrm{Al}_{2} \mathrm{O}_{3} .2 \mathrm{SiO}_{2}\left(29.2 \% \mathrm{CaO}, 39 \% \mathrm{Al}_{2} \mathrm{O}_{3}, 31.8 \% \mathrm{SiO}_{2}\right) ; 1380^{\circ} \pm 5^{0}$. 
Tabelle 11.

Schmelzpunkt des Eutektikums 2 für $\mathrm{CaSiO}_{3}+\mathrm{CaO} \cdot \mathrm{Al}_{2} \mathrm{O}_{3}$. $2 \mathrm{SiO}_{2}+\mathrm{SiO}_{2}$, bestimmt aus verschiedenen Zusammensetzungen.

\begin{tabular}{|c|c|c|c|c|c|c|c|c|c|c|c|}
\hline $\begin{array}{c}\text { Zusammensetzung } \\
\text { in } \\
\text { Gew.- } \%\end{array}$ & $\begin{array}{c}\mathrm{CaO} \\
\mathrm{Al}_{2} \mathrm{O}_{3} \\
\mathrm{SiO}_{2}\end{array}$ & $\begin{array}{r}25 \\
5 \\
70 \\
\end{array}$ & $\begin{array}{l}20 \\
10 \\
70 \\
\end{array}$ & $\begin{array}{l}15 \\
15 \\
70 \\
\end{array}$ & $\begin{array}{r}30 \\
5 \\
65 \\
\end{array}$ & $\begin{array}{l}25 \\
10 \\
65 \\
\end{array}$ & $\begin{array}{l}20 \\
15 \\
65 \\
\end{array}$ & $\begin{array}{l}15 \\
20 \\
65 \\
\end{array}$ & $\begin{array}{r}35 \\
5 \\
60 \\
\end{array}$ & $\begin{array}{l}30 \\
10 \\
60 \\
\end{array}$ & $\begin{array}{l}25 \\
15 \\
60 \\
\end{array}$ \\
\hline Schmelztemperatur & & $\begin{array}{l}1171 \\
1172 \\
1169\end{array}$ & $\begin{array}{r}1183 \\
1174 \\
1183 \\
.\end{array}$ & $\begin{array}{l}1174 \\
1175 \\
1175 \\
1174 \\
1174 \\
1173\end{array}$ & $\left|\begin{array}{l}1165 \\
1165 \\
1165 \\
1165\end{array}\right|$ & $\begin{array}{l}1175 \\
1175 \\
1174\end{array}$ & $\begin{array}{l}1173 \\
1177\end{array}$ & $\begin{array}{l}1173 \\
1175 \\
1173 \\
1169\end{array}$ & $\begin{array}{l}1170 \\
1171 \\
1170\end{array}$ & $\begin{array}{l}1175 \\
1169 \\
1173 \\
1172 \\
1171\end{array}$ & $\begin{array}{l}1172 \\
1173 \\
1171\end{array}$ \\
\hline Mittel & & 1171 & 1180 & 1174 & 1165 & 1175 & 1175 & 1172 & 1170 & 1172 & 1172 \\
\hline $\begin{array}{c}\text { Zusammensetzung } \\
\text { in } \\
\text { Gew. }-\%\end{array}$ & $\begin{array}{c}\mathrm{CaO} \\
\mathrm{Al}_{3} \mathrm{O}_{9} \\
\mathrm{SiO}_{2}\end{array}$ & $\begin{array}{l}20 \\
20 \\
60\end{array}$ & $\begin{array}{l}15 \\
25 \\
60 \\
\end{array}$ & $\begin{array}{r}40 \\
5 \\
55 \\
\end{array}$ & $\begin{array}{l}35 \\
10 \\
55\end{array}$ & $\begin{array}{l}25 \\
20 \\
55\end{array}$ & $\begin{array}{l}20 \\
25 \\
55\end{array}$ & \begin{tabular}{|l|}
23.5 \\
14.5 \\
62
\end{tabular} & $\begin{array}{l}30 \\
10 \\
60\end{array}$ & $\begin{array}{l}35 \\
10 \\
55\end{array}$ & \\
\hline Schmelztemperatur & & $\begin{array}{l}1167 \\
1171 \\
1172 \\
1173\end{array}$ & $\begin{array}{l}1168 \\
1163 \\
1167 \\
1167 \\
1166 \\
\end{array}$ & 116 & $\left|\begin{array}{l}1171 \\
1174 \\
1174\end{array}\right|$ & $\begin{array}{l}1176 \\
1173\end{array}$ & $\begin{array}{l}1173 \\
1178 \\
1178\end{array}$ & & hrect & $\frac{1170}{\text { kung }}$ & \\
\hline Mittel & & 17 & 16 & 117 & 177 & & 17 & 1 & & 117 & \\
\hline
\end{tabular}

Punkt 12 ist das Eutektikum von $2 \mathrm{CaO} . \mathrm{Al}_{2} \mathrm{O}_{3} \cdot \mathrm{SiO}_{2}, 3 \mathrm{CaO}$. $5 \mathrm{Al}_{2} \mathrm{O}_{3}$ und $\mathrm{CaO} . \mathrm{Al}_{2} \mathrm{O}_{3}\left(37.5 \%\right.$ CaO, $\left.53.25 \% \quad \mathrm{Al}_{2} \mathrm{O}_{3}, 9.25 \% \quad \mathrm{SiO}_{2}\right)$; $1505^{0} \pm 5^{0}$.

Punkt 14 ist das Eutektikum für $\mathrm{Ca}_{2} \mathrm{SiO}_{4}, 5 \mathrm{CaO} .3 \mathrm{Al}_{2} \mathrm{O}_{3}$ und $\mathrm{CaO} . \mathrm{Al}_{2} \mathrm{O}_{3}\left(49.5 \% \mathrm{CaO}, 43.7 \% \mathrm{Al}_{2} \mathrm{O}_{3}, 6.8 \%, \mathrm{SiO}_{2}\right) ; 1335^{\circ} \pm 5^{0}$.

Punkt 15 ist das Eutektikum für $\mathrm{Ca}_{2} \mathrm{SiO}_{4}, 5 \mathrm{CaO} .3 \mathrm{Al}_{2} \mathrm{O}_{3}$ und $3 \mathrm{CaO} . \mathrm{Al}_{2} \mathrm{O}_{3}\left(52 \% \mathrm{CaO}, 41.2 \% \mathrm{Al}_{2} \mathrm{O}_{3}, 6.8 \% \mathrm{SiO}_{2}\right) ; 1335^{\circ} \pm 5^{0}$.

Die einzige zufriedenstellende Methode zur Bestimmung der Schmelzpunkte der Eutektika 14 und 15 ist das Abschreckungsverfahren. Bei dem Versuch, die Temperaturen des Schmelzens dieser beiden Eutektika durch Erhitzungskurven zu bestimmen, erhielt man keine zufriedenstellenden Ergebnisse und zwar aus den folgenden Gründen:

1. $5 \mathrm{CaO} .3 \mathrm{Al}_{2} \mathrm{O}_{3}$ sowohl im reinen Zustand wie in ternären Gemischen, siedet auf, wenn man es fein gepulvert auf eine Temperatur erhitzt, bei dor das Schmelzen beginnt; und es siedet um so heftiger, je höher die Temperatur steigt. Der größte Teil einer Beschickung von $20 \mathrm{~g}$ siedet aus einem Platintiegel von $100 \mathrm{ccm}$ fort. Wenn man große Stücke dieser Verbindung oder ternäre Gemische, in denen eine beträchtliche Menge dieser Verbindung enthalten ist, 
zum Schmelzen bringt, so tritt diese Erscheinung nicht auf. Die Ursache für dieses Sieden haben wir nicht vollständig untersucht; wahrscheinlich ist sie auf absorbiertes Gas zurückzuführen, das erst beim Schmelzen entweicht. Wir wissen nur, daß dieses Sieden Störungen verursacht, wenn es bei einer Beschickung auftritt, von der wir die Erhitzungskurve zu ermitteln wünschen. 2. Die instabile Form von $5 \mathrm{CaO} .3 \mathrm{Al}_{2} \mathrm{O}_{3}$ erscheint und verschwindet in Schmelzen des ternären und des binären Systemes. Hierdurch werden unbestimmte und unregelmäBige Knicke beim Erhitzen hervorgerufen, die bei fast jeder Temperatur auftreten können. Aus diesen Gründen lassen sich zufriedenstellende Knicke auf den Erhitzungskurven für diese beiden Eutektika nicht erhalten, ausgenommen für die Gemische, die nur eine kleine Menge von $5 \mathrm{CaO} .3 \mathrm{Al}_{2} \mathrm{O}_{3}$ enthalten. In diesen Fällen aber ist nur so wenig Eutektikum vorhanden, daß der Knick nicht sehr scharf ausgeprägt ist.

Nichteutektische Quintupelpunkte. Die Daten für die nichteutektischen Quintupelpunkte sind in derselben Weise angegeben, wie für die eutektischen Punkte, d. h. es ist die vollständige Beobachtungsreihe, aus der die Temperatur eines Punktes abgeleitet ist, mitgeteilt, jedoch nur ein Wert (das Mittel einer großen Zahl von Bestimmungen) für jeden der anderen Punkte. Die Nummer, mit der jeder Quintupelpunkt bezeichnet ist, entspricht der Nummer von Fig. 6.

Tabelle 12.

Schmelztemperatur: Quintupelpunkt 4 für $\beta-2 \mathrm{CaO} . \mathrm{SiO}_{2}, 2 \mathrm{CaO}$. $\mathrm{Al}_{2} \mathrm{O}_{3} . \mathrm{SiO}_{2}$ und $3 \mathrm{CaO} .2 \mathrm{SiO}_{2}$, bestimmt aus verschiedenen Gemischen.

\begin{tabular}{|c|c|c|c|c|c|c|}
\hline $\begin{array}{c}\text { Zusammensetzung } \\
\text { in } \\
\text { Gew.- } \%\end{array}$ & $\begin{array}{l}\mathrm{CaO} \\
\mathrm{Al}_{2} \mathrm{O}_{3} \\
\mathrm{SiO}_{2} \\
\end{array}$ & $\begin{array}{r}60 \\
5 \\
35 \\
\end{array}$ & $\begin{array}{l}55 \\
10 \\
35 \\
\end{array}$ & $\begin{array}{l}45 \\
30 \\
25\end{array}$ & $\begin{array}{r}55 \\
5 \\
40 \\
\end{array}$ & $\begin{array}{l}48.5 \\
12 \\
39.5\end{array}$ \\
\hline Schmelztemperatur & & $\begin{array}{l}1333 \\
1334 \\
1334 \\
1332\end{array}$ & $\begin{array}{l}1337 \\
1337 \\
1338 \\
1339 \\
1340 \\
1341 \\
\end{array}$ & $\begin{array}{l}1334 \\
1337 \\
1337 \\
1336 \\
1336\end{array}$ & $\begin{array}{l}1333 \\
1336 \\
1333\end{array}$ & $\begin{array}{c}1337 \\
\mathrm{Ab}- \\
\text { schreckung }\end{array}$ \\
\hline Mittel & & 1333 & 1338 & 1336 & 1333 & 1337 \\
\hline
\end{tabular}

Punkt 4 ist der Quintupelpunkt für $2 \mathrm{CaO} \cdot \mathrm{Al}_{2} \mathrm{O}_{3} \cdot \mathrm{SiO}_{2}, \beta-\mathrm{Ca}_{2} \mathrm{SiO}_{4}$ und $3 \mathrm{CaO}_{2} 2 \mathrm{SiO}_{2}\left(48.2 \% \mathrm{CaO}, 11.9 \% \mathrm{Al}_{2} \mathrm{O}_{3}, 39.9 \% \mathrm{SiO}_{2}\right)$. Seine Schmelztemperatur $1335^{\circ} \pm 5^{0}$ hängt ab von den für verschiedene Gemisehe erhaltenen Werten, wie aus Tabelle 12 hervorgeht. 
Punkt 3 ist der Quintupelpunkt für $\alpha-2 \mathrm{CaO} . \mathrm{SiO}_{2}, \beta-2 \mathrm{CaO} . \mathrm{SiO}_{2}$ und $3 \mathrm{CaO} .2 \mathrm{SiO}_{2}\left(53.0^{\circ} \% \mathrm{CaO}, 4.8^{\circ} \mathrm{Al}_{2} \mathrm{O}_{3}, 42.8 \% \mathrm{SiO}_{2}\right) ; 1415^{\circ} \pm 5^{0}$.

Punkt 8 ist der Quintupelpunkt von $\alpha-2 \mathrm{CaO} . \mathrm{SiO}_{2}, \beta-2 \mathrm{CaO} . \mathrm{SiO}_{2}$ und $2 \mathrm{CaO} \cdot \mathrm{Al}_{2} \mathrm{O}_{3} \cdot \mathrm{SiO}_{2}\left(49 \% \mathrm{CaO}, 14.4 \% \mathrm{Al}_{2} \mathrm{O}_{3}, 36.6 \% \mathrm{SiO}_{2}\right) ; 1415^{0}$ $\pm 5^{0}$.

Punkt 9 ist der Quintupelpunkt von $\mathrm{Al}_{2} \mathrm{O}_{3}, \mathrm{CaO} \cdot \mathrm{Al}_{2} \mathrm{O}_{3} \cdot 2 \mathrm{SiO}$ und $\mathrm{Al}_{2} \mathrm{O}_{3} \cdot \mathrm{SiO}_{2}\left(15.6 \% \mathrm{CaO}, 36.5^{\circ} \mathrm{Al}_{2} \mathrm{O}_{3}, 47.9^{\circ} \% \mathrm{SiO}_{2}\right) ; 1512^{\circ} \pm 5^{0}$.

Punkt 10 ist der Quintupelpunkt von $\mathrm{SiO}_{2}$ (Tridymit), $\mathrm{SiO}_{2}$ (Cristobalit) und $\mathrm{Al}_{2} \mathrm{O}_{3} . \mathrm{SiO}_{2}$ (Sillimanit). (6.6\% $\mathrm{CaO}, 17.6 \% \mathrm{Al}_{2} \mathrm{O}_{3}$, $\left.75.8 \% \quad \mathrm{SiO}_{2}\right) ; 1470^{\circ}$.

Punkt 11 ist der Quintupelpunkt für $3 \mathrm{CaO} .5 \mathrm{Al}_{2} \mathrm{O}_{3}, 2 \mathrm{CaO} . \mathrm{Al}_{2} \mathrm{O}_{3}$. $\mathrm{SiO}_{2}$ und $\mathrm{Al}_{2} \mathrm{O}_{3}\left(31.2 \% \mathrm{CaO}, 44.5 \% \mathrm{Al}_{2} \mathrm{O}_{3}, 24.3 \% \mathrm{SiO}_{2}\right) ; 1475^{0} \pm 5^{0}$.

Punkt 13 ist der Quintupelpunkt für $2 \mathrm{CaO} \cdot \mathrm{Al}_{2} \mathrm{O}_{3} . \mathrm{SiO}_{2}, 2 \mathrm{CaO}$. $\mathrm{SiO}_{2}$ und $\mathrm{CaO} . \mathrm{Al}_{2} \mathrm{O}_{3}\left(48.3 \% \mathrm{CaO}, 42 \% \mathrm{Al}_{2} \mathrm{O}_{3}, 9.7 \% \mathrm{SiO}_{2}\right) ; 1380^{0} \pm 5^{0}$.

Punkt 16 ist der Quintupelpunkt für $3 \mathrm{CaO} . \mathrm{Al}_{2} \mathrm{O}_{3}, 3 \mathrm{CaO} . \mathrm{SiO}_{2}$ und $2 \mathrm{CaO} \mathrm{SiO}_{2}\left(58.3 \% \mathrm{CaO}, 33.0 \% \mathrm{Al}_{2} \mathrm{O}_{3}, 8.7 \% \mathrm{SiO}_{2}\right) ; 1455^{\circ} \pm 5^{0}$.

Punkt 17 ist der Quintupelpunkt für $\mathrm{CaO}, 3 \mathrm{CaO} \cdot \mathrm{Al}_{2} \mathrm{O}_{3}$ und $3 \mathrm{CaO} . \mathrm{SiO}_{2}\left(59.7 \% \mathrm{CaO}, 32.8 \% \mathrm{Al}_{2} \mathrm{O}_{3}, 7.5^{\%} \quad \mathrm{SiO}_{2}\right) ; 1470^{\circ} \pm 5^{0}$.

Punkt 18 ist der. Quintupelpunkt für $\mathrm{CaO}, 2 \mathrm{CaO} . \mathrm{SiO}_{2}$ and $3 \mathrm{CaO} . \mathrm{SiO}_{2}\left(68.4 \% \mathrm{CaO}, 9.2 \% \quad \mathrm{Al}_{2} \mathrm{O}_{3}, 22.4 \% \quad \mathrm{SiO}_{2}\right) ; 1900^{\circ} \pm 20^{\circ}$.

Punkt 18 wurde ermittelt durch Extrapolation auf den Grenzkurven 16-18 und 17-18. Die Richtung der unteren Teile dieser Kurven - bei Temperaturen bis $1600^{\circ}$ - bestimmte man durch Abschreckung; die Temperaturen der oberen Teile liegt außerhalb des Gebietes von Temperaturmessungen, so daß weder die Lage, noch die Temperatur von Punkt 18 sehr genau bestimmt ist. Punkt 18 ist die Dissoziationstemperatur vom $3 \mathrm{CaO} . \mathrm{SiO}_{2}$.

Punkt 19 ist der Quintupelpunkt für $\alpha-2 \mathrm{CaO} . \mathrm{SiO}_{2}, \beta-2 \mathrm{CaO} . \mathrm{SiO}_{2}$ und $3 \mathrm{CaO} \mathrm{Al}_{2} \mathrm{O}_{3}\left(54.5 \% \mathrm{CaO}, 38 \% \mathrm{Al}_{2} \mathrm{O}_{3}, 7.5 \% \mathrm{SiO}_{2}\right) ; 1390^{\circ}$.

Punkt 20 ist der Quintupelpunkt von $\alpha-2 \mathrm{CaO} . \mathrm{SiO}_{2}, \beta-2 \mathrm{CaO} . \mathrm{SiO}_{2}$ und $2 \mathrm{CaO} . \mathrm{Al}_{2} \mathrm{O}_{3} . \mathrm{SiO}_{2}\left(48.7 \% \mathrm{CaO}, 39.3 \% \mathrm{Al}_{2} \mathrm{O}_{3}, 12 \% \mathrm{SiO}_{2}\right) ; 1430^{\circ}$.

\section{Zusammenfassung: Das Konzentrations-Temperatur-Modell.}

Auf den voraufgehenden Seiten haben wir die Gleichgewichtstemperaturen, soweit sie sich mit unserem Apparat bestimmen ließen, für die drei binären Systeme und für das ternäre System mitgeteilt; mit anderen Worten, wir haben die Temperaturen angegeben, die den Quintupelpunkten, den Grenzkurven und den Feldern entsprechen. Durch Interpolationen aus den festgelegten Schmelztemperaturen können wir Linien konstanter Temperatur 
oder Isothermen ableiten; dies ist erfolgt für Zwischenräume von je $100^{\circ}$ von $1200^{\circ}$ bis $1700^{\circ}$, und die entstehende Zeichnung ist in Fig. 8 dargestellt, die überdies die Schmelztemperaturen und die Zusammensetzungen jeder Komponente, Verbindung, die Quadrupelpunkte und die Quintupelpunkte enthält.

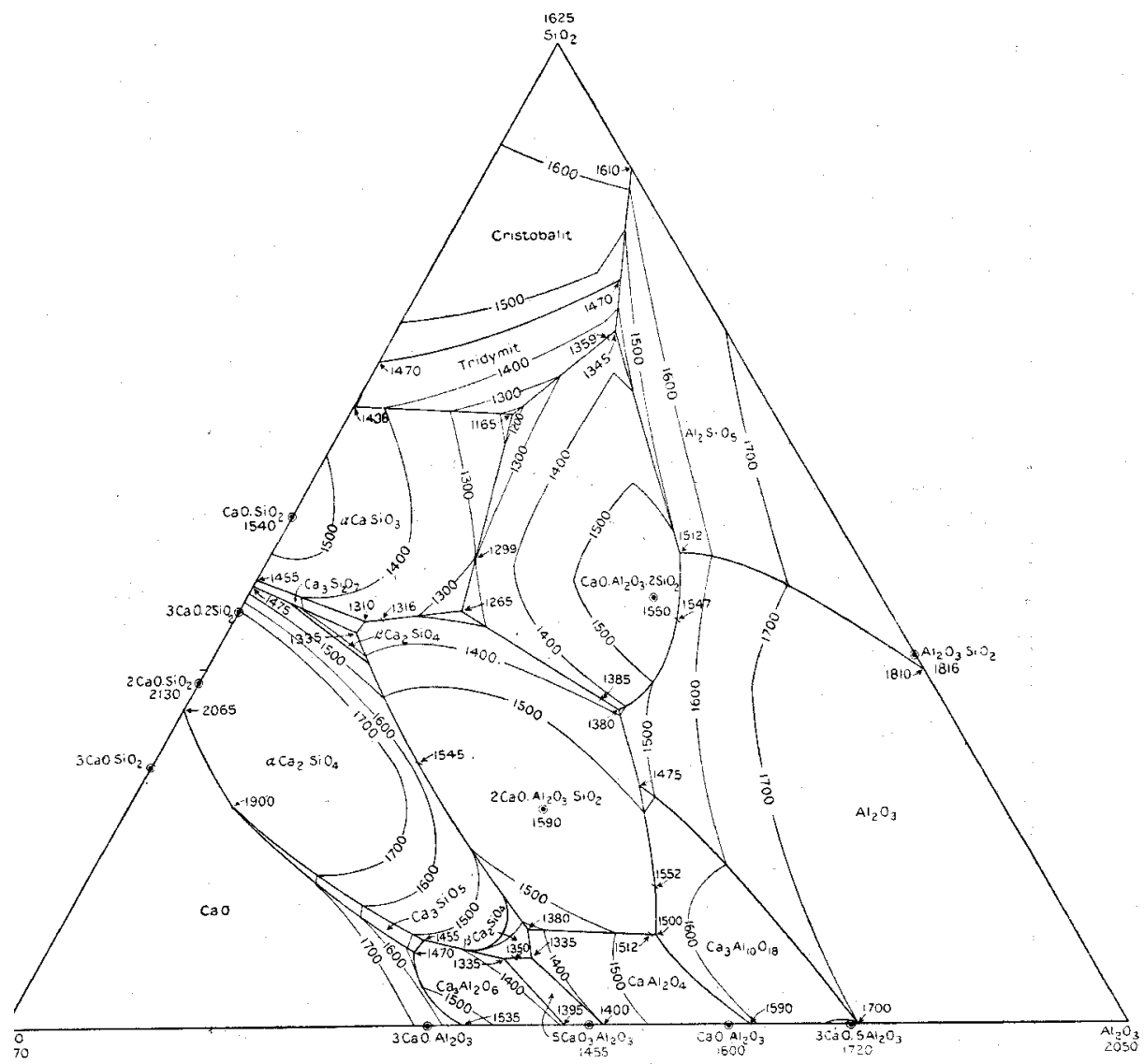

Fig. 8.

Projektion des Temperatur-Konzentrations-Diagrammes des Systemes $\mathrm{CaO}-\mathrm{Al}_{2} \mathrm{O}_{3}-\mathrm{SiO}_{2}$ mit Isothermen und Schmelztemperaturen der Verbindungen und invarianten Punkte.

Wenn man das Konzentrations-Temperatur-Diagramm (Fig. 7) für jede.Grenzkurve (Fig. 6) senkrecht auf der Ebene der entsprechenden Projektion jeder Grenzkurve (Fig. 6) errichtet, so stellt man ein räumliches Konzentrations-Temperatur-Modell her, dessen Oberfläche die Schmelztemperaturen aller ternären Mischungen von 
$\mathrm{CaO}-\mathrm{Al}_{2} \mathrm{O}_{3}-\mathrm{SiO}_{2}$ darstellt. Eine eingehende Beschreibung des Verfahrens zur Herstellung eines solchen räumlichen Modelles ist in einer Fußnote auf Seite 229 gegeben. Die Photographien eines auf diese Weise gewonnenen räumlichen Modelles sind in den Figg. 9-11 dargestellt. Fig. 9 ist aufgenommen von der $\mathrm{SiO}_{2}$-Ecke und zeigt die

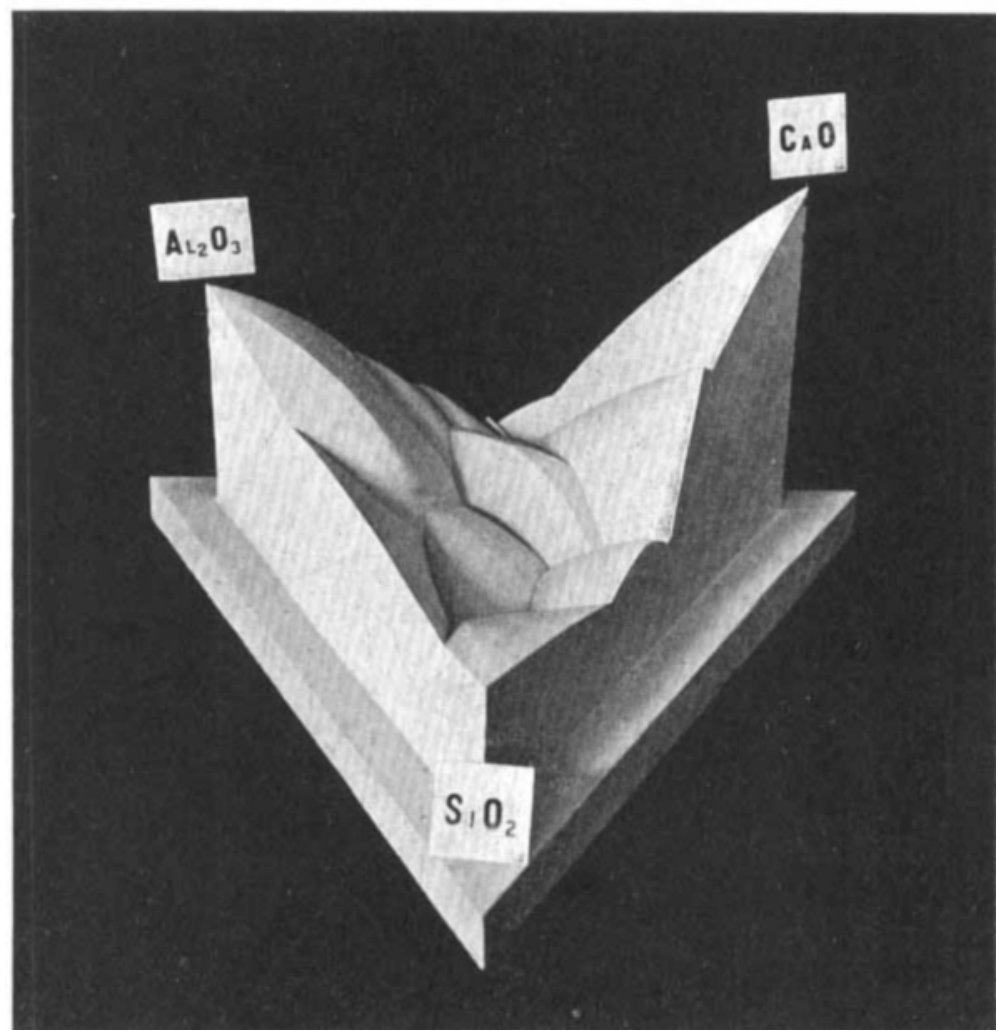

Fig. 9. Photographie des räumlichen Modelles des Konzentrations-TemperaturDiagrammes des ternären Systems; das Bild läßt die Beziehungen der binären Systeme $\mathrm{CaO}-\mathrm{SiO}_{2}$ und $\mathrm{Al}_{2} \mathrm{O}_{3}-\mathrm{SiO}_{2}$ zum ternären System erkennen.

binären Systeme $\mathrm{CaO}-\mathrm{SiO}_{2}$ und $\mathrm{Al}_{2} \mathrm{O}_{3}-\mathrm{SiO}_{2}$ sowie den Verlauf der ternären Felder, die von diesen beiden binären Systemen ausgehen. In ähnlicher Weise zeigt Fig. 10 die binären Systeme von $\mathrm{CaO}-\mathrm{Al}_{2} \mathrm{O}_{3}$ und $\mathrm{Al}_{2} \mathrm{O}_{3}-\mathrm{SiO}_{2}$ sowie ihre Beziehung zum ternären System. Fig. 11 ist eine Photographie, die das räumliche Modell direkt von oben zeigt und die relativen Lagen und den Verlauf der verschiedenen Felder erkennen läßt. 
Wie man aus den drei Photographien ersieht, hat das Konzentrations-Temperatur-Modell des ternären Systemes $\mathrm{CaO}-\mathrm{Al}_{2} \mathrm{O}_{3}-\mathrm{SiO}_{2}$ das allgemeine Aussehen eines Gebirges; die Berggipfel sind die Schmelzpunkte der Komponenten oder der bei ihrem Schmelzpunkt stabilen Verbindungen; der kontinuierliche Abfall auf den Seiten

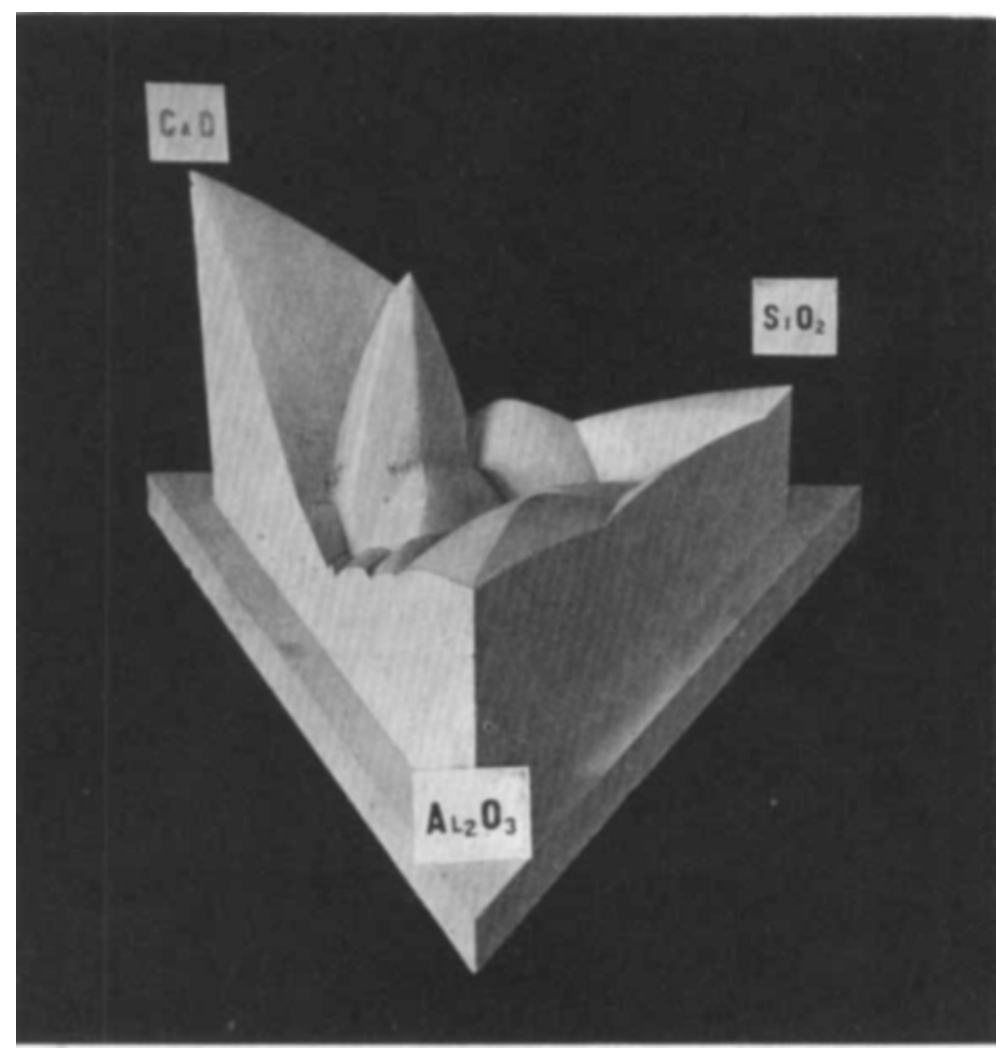

Fig. 10. Photographie des räumlichen Modelles; das Bild zeigt die Beziehung der binären Systeme $\mathrm{CaO}-\mathrm{Al}_{2} \mathrm{O}_{3}$ und $\mathrm{Al}_{2} \mathrm{O}_{3}-\mathrm{SiO}_{2}$ zum ternären System.

cines jeden Berges stellt die Schmelztemperaturen einer stabilen Verbindung oder Komponente im ternären System dar; wenn eine Unterbrechung im kontinuierlichen Abfall auf der Seite eines Gipfels stattfindet, dann entspricht der Abfall von dieser Unterbrechung bis zum Fuß des Berges den Schmelztemperaturen einer instabilen Verbindung in den ternären Lösungen; die Täler zwischen den Bergen stellen die Grenzkurven Z. anorg. Chem. Bd. 9?. 
dar; die Punkte, an denen sich die Flüsse in drei Tälern unter Bildung eines Sees vereinigen, sind eutektische Punkte; wenn sich aber zwei Flüsse an einem Punkt vereinigen und dann einen einzigen Strom bilden, dann ist dieser Punkt ein Quintupelpunkt, aber ohne eutektischen Charakter.

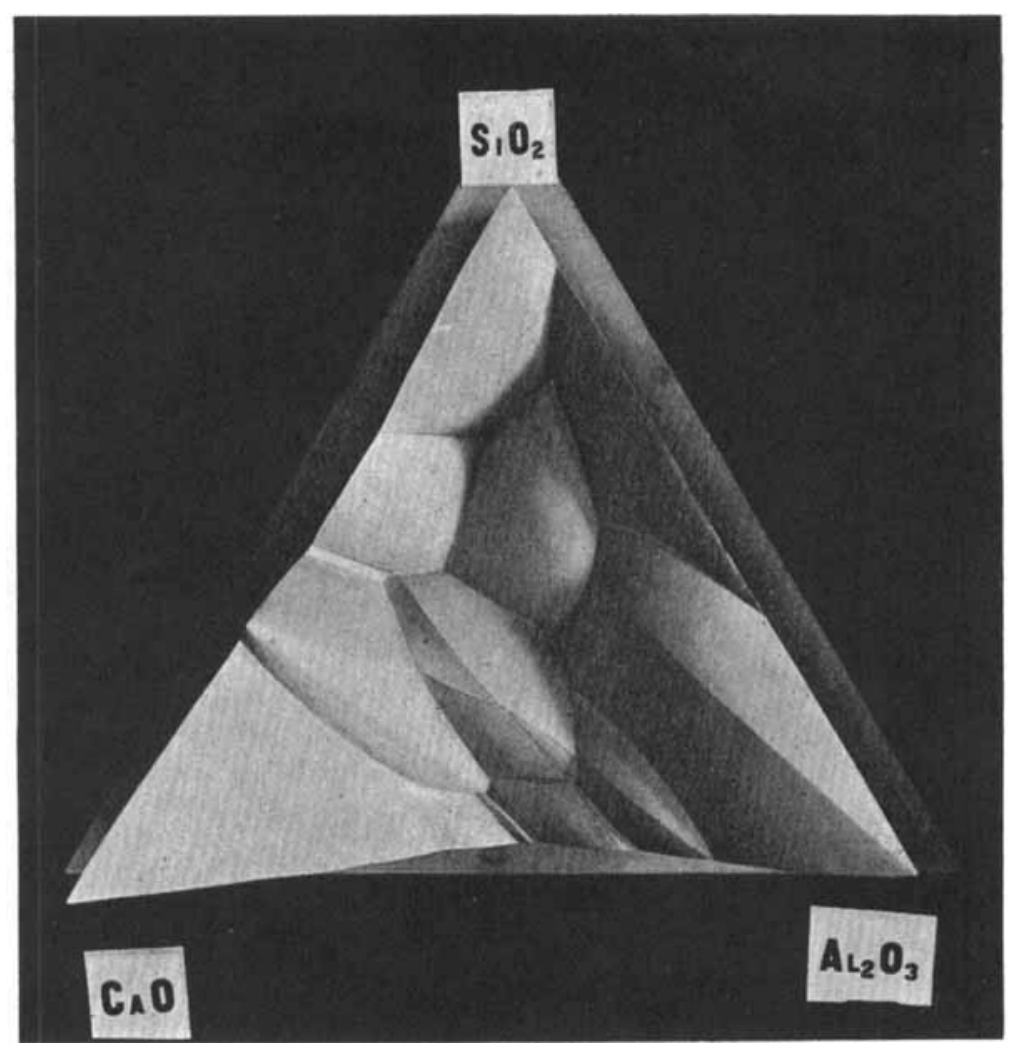

Fig. 11. Photographie des räumlichen Modelles von oben; das Bild zeigt die Beziehưngen der verschiedenen Felder.

\section{Einige allgemeine Betrachtungen über das System $\mathrm{CaO}-\mathrm{Al}_{2} \mathrm{O}_{3}-\mathrm{SiO}_{2}$.}

In dem Vorausgehenden haben wir die verschiedenen Punkte, Kurven und Existenzgebiete getrennt aufgenommen; wir werden nun dazu übergehen, ihre allgemeineren Beziehungen zu besprechen. Hierbei wird es zweckmäßig sein, jede Komponente mit ihrem Anfangsbuchstaben zu bezeichnen, also $C=\mathrm{CaO}, A=\mathrm{Al}_{2} \mathrm{O}_{3}, S=\mathrm{SiO}_{2}$. Hierdurch vereinfacht sich die Bezeichnung der Verbindungen, es 
wird demnach $C S=\mathrm{CaO} . \mathrm{SiO}_{2}, C A S_{2}=\mathrm{CaO} . \mathrm{Al}_{2} \mathrm{O}_{3} .2 \mathrm{SiO}_{2}, C_{3} A_{5}=$ $3 \mathrm{CaO} .5 \mathrm{Al}_{2} \mathrm{O}_{3}$ usw.

\section{Die verschiedenen binären Systeme im ternären System.}

Die Beziehungen sind zu erkennen aus einer Untersuchung der Zeichnung von Fig. 12. In dieser Zeichnung sind außer den Grenz-

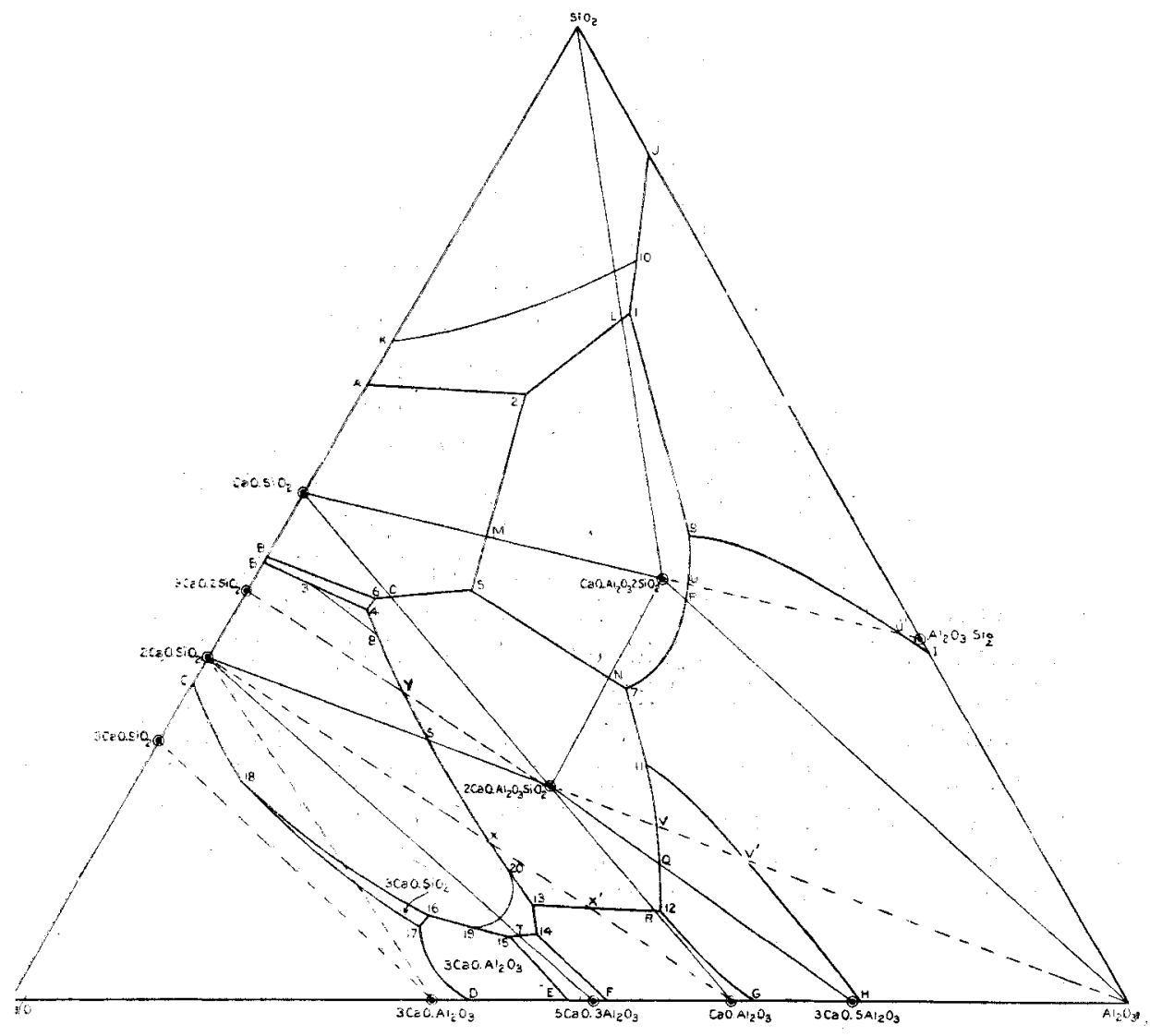

Fig. 12.

Diagramm des ternären Systemes, welches die verschiedenen in ihm vorhandenen binären Systeme zeigt.

kurven gerade Linien eingetragen, die die Zusammensetzungen eines jeden Paares von Verbindungen vereinigen, deren Felder eine gemeinschaftliche Grenzkurve besitzen; jede von diesen geraden Linien, die alle möglichen Gemische der beiden Verbindungen darstellt, 
deren Zusammensetzungen sie vereinigt, ist eine horizontale Projektion der Schmelzkurve dieser Gemische in ihren Lösungen. Ein Blick auf die Zeichnung lehrt folgendes : a) Einige der geraden Linien; die voll ausgezogen sind, liegen ganz in den Feldern der beiden Stoffe deren Zusammensetzungen sie verbinden; b) andere, die gestrichelt gezeichnet sind, liegen nur zum Teil in diesen beiden Feldern; $\mathrm{c}$ ) noch andere liegen zum Teil nur in einem dieser Felder und d) einige Linien liegen auch gänzlich außerhalb dieser Felder.

a) Die gerade Linie liegt ganz in den Feldern der beiden Stoffe, deren Zusammensetzung sie verbindet. Als Beispiel einer solchen Linie betrachten wir die Gerade, die die Zusammensetzungen der beiden Stoffe $C S\left(\mathrm{CaO}_{\mathrm{SiO}}\right)$ und $C A S_{2}$ $\left(\mathrm{CaO} . \mathrm{Al}_{2} \mathrm{O}_{3} .2 \mathrm{SiO}_{2}\right)$ verbindet. Diese Linie schneidet die ternäre Grenze 5-2 für $C S$ und $C A S_{2}$ bei $M$. Der Teil dieser Linie, welcher $C S$ und $M$ vereinigt, ist dann die Schmelzkurve von $C S$ in Lösungen mit $C A S_{2}$, und der Teil der Linie, der $C A S_{2}$ und $M$ verbindet, ist. die Schmelzkurve von $C A S_{2}$ in Lösungen mit $C S . M$ ist derjenige Punkt, bei dem $C S$ und $C A S_{2}$ gemeinsam schmelzen; es ist ein eutektischer Punkt. Das System $C S-C A S_{2}$ ist demnach ein einfaches binäres eutektisches System, wenn man die Verbindungen $C S$ und $C A S_{2}$ als Komponenten betrachtet. Acht solcher binärer Systeme, die durch ausgezogene Linien in Fig. 12 dargestellt werden, sind vorhanden. Zwei dieser Systeme $C S-C_{2} A S$ und $C_{2} A S-C A$ liegen auf derselben geraden Linie und können behandelt werden als ein einziges binäres System, in dem sich eine stabile Verbindung $C_{\mathbf{2}} A S$ bildet. Die Daten für die wichtigen Punkte dieser Systeme sind in den Tabellen 12 bis 20 zusammengestellt; die entsprechenden Temperatur-Konzentrations-Diagramme findet man in den Figg. 13 bis 16. Die Abszisse oder Konzentrationslinie für jedes dieser Systeme ist direkt aus Fig. 12 entnommen und die Ordinaten stellen die Temperaturen dar.

Tabelle 13.

Schmelztemperatur des binären Systemes $\mathrm{SiO}_{2}-\mathrm{CaO} \cdot \mathrm{Al}_{2} \mathrm{O}_{3} \cdot 2 \mathrm{SiO}_{2}$.

\begin{tabular}{c|c|c|cc|c}
\hline \hline & & $\begin{array}{c}\text { Komponente } \\
\mathrm{SiO}_{2}\end{array}$ & Eutektikum & $\begin{array}{c}\text { Komponente } \\
\mathrm{CaO} . \mathrm{Al}_{2} \mathrm{O}_{3} .2 \mathrm{SiO}_{2}\end{array}$ \\
\hline \hline Zusammensetzung & $\mathrm{CaO}$ & 0.0 & 10.5 & 19.0 & 20.09 \\
in & $\mathrm{Al}_{2} \mathrm{O}_{3}$ & 0.0 & 19.5 & 34.9 & 36.63 \\
Gew.-\% & $\mathrm{SiO}_{2}$ & 100.0 & 70.0 & 46.1 & 43.28 \\
\hline \hline Schmelztemperatur & & 1625 & 1359 & 1542 & 1550
\end{tabular}


Ternäres System: Calciumoxyd-Aluminiumoxyd-Silicium-2-oxyd. 261

Tabelle 14 .

Schmelatemperatur des binären Systemes $\mathrm{CaO} \cdot \mathrm{SiO}_{2}-\mathrm{CaO} \cdot \mathrm{Al}_{2} \mathrm{O}_{3} \cdot 2 \mathrm{SiO}_{2}$.

\begin{tabular}{c|c|c|c|c|c|c}
\hline & & $\begin{array}{c}\text { Komponente } \\
\mathrm{CaO} . \mathrm{SiO}_{2}\end{array}$ & \multicolumn{2}{|c|}{ Eutektikum } & $\begin{array}{c}\text { Komponente } \\
\mathrm{CaO} . \mathrm{Al}_{2} \mathrm{O}_{3} .2 \mathrm{SiO}_{2}\end{array}$ \\
\hline \begin{tabular}{c|c|c|c|c|c} 
Zusammensetzung \\
in
\end{tabular} & $\mathrm{CaO}$ & 48.2 & 41.4 & 34.1 & $\mathbf{2 7}$ & 20.9 \\
Gew.-\% $\%$ & $\mathrm{Al}_{2} \mathrm{O}_{3}$ & 0.0 & 9.0 & 18.6 & 27.5 & 36.63 \\
\hline $\mathrm{SiO}_{2}$ & 51.8 & 49.6 & 47.3 & 45.5 & 43.28 \\
\hline Schmelztemperatur & & 1540 & 1436 & 1299 & 1484 & 1550
\end{tabular}

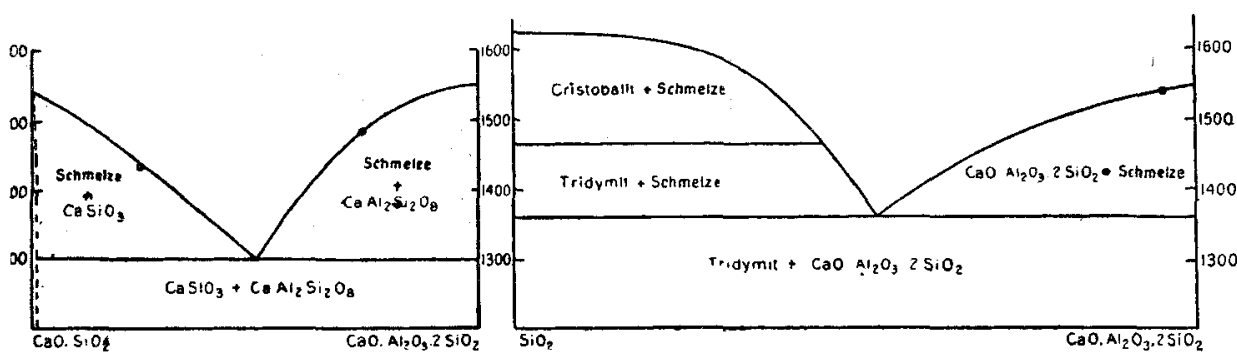

Fig. 13. Temperatur-Konzentrations-Diagramm der binären Systeme $\mathrm{CaO} . \mathrm{SiO}_{2}-\mathrm{CaO} . \mathrm{Al}_{2} \mathrm{O}_{3} .2 \mathrm{SiO}_{2}$ und $\mathrm{SiO}_{2}-\mathrm{CaO} . \mathrm{Al}_{2} \mathrm{O}_{3} \cdot 2 \mathrm{SiO}_{2}$.

Tabelle 15.

Schmelztemperatur des binären Systemes $\mathrm{CaO} \cdot \mathrm{Al}_{2} \mathrm{O}_{3} \cdot 2 \mathrm{SiO}_{2}-\mathrm{Al}_{2} \mathrm{O}_{3}$.

\begin{tabular}{c|c|c|c|c}
\hline & $\begin{array}{c}\text { Komponente } \\
\mathrm{CaO} . \mathrm{Al}_{2} \mathrm{O}_{3} \cdot 2 \mathrm{SiO} \mathrm{O}_{2}\end{array}$ & Eutektikum & $\begin{array}{c}\text { Komponente } \\
\mathrm{Al}_{2} \mathrm{O}_{3}\end{array}$ \\
\hline \hline Zusammensetzung & $\mathrm{CaO}$ & 20.09 & 19.3 & 0 \\
in & $\mathrm{Al}_{2} \mathrm{O}_{3}$ & 36.63 & 39.3 & 100 \\
Gew.-\% & $\mathrm{SiO}_{2}$ & 43.28 & 41.4 & 0 \\
\hline Schmelztemperatur & & 1550 & 1547 & 2050
\end{tabular}

Tabelle 16.

Schmelztemperatur des binären Systemes $2 \mathrm{CaO} \cdot \mathrm{Al}_{2} \mathrm{O}_{3} \cdot \mathrm{SiO}_{2}-2 \mathrm{CaO} \cdot \mathrm{SiO}_{2}$.

\begin{tabular}{c|c|c|c|c|c|c|c|c}
\hline \hline & $\begin{array}{c}\text { Komponente } \\
2 \mathrm{CaO} \mathrm{Al}_{2} \mathrm{O}_{3}\end{array}$ & \multicolumn{3}{c|}{ Eutektikum } & & \multicolumn{2}{c}{$\begin{array}{c}\text { Kom- } \\
\text { ponente } \\
\mathrm{Ca}_{2} \mathrm{SiO}_{4}\end{array}$} \\
\hline \hline Zusammensetzung & $\mathrm{CaO}$ & 40.83 & 42.3 & 45.8 & 46.6 & 49.6 & 50.7 & 65 \\
in & $\mathrm{Al}_{2} \mathrm{O}_{3}$ & 37.19 & 35.0 & 29.5 & 28.3 & 23.7 & 22.0 & 0 \\
Gew.-\% & $\mathrm{SiO}_{2}$ & 21.98 & 22.7 & 24.7 & 25.1 & 26.7 & 27.3 & 35 \\
\hline Schmelztemperatur & & 1590 & 1588 & 1574 & 1564 & 1545 & $1625 \mid$ & 2130
\end{tabular}


Tabelle 17.

Schmelztemperaturd. binär. Systemes $\mathrm{CaO} \cdot \mathrm{Al}_{2} \mathrm{O}_{3} \cdot 2 \mathrm{SiO}_{2}-2 \mathrm{CaO} \cdot \mathrm{Al}_{2} \mathrm{O}_{3} \cdot \mathrm{Si}_{2} \mathrm{O}$.

\begin{tabular}{|c|c|c|c|c|c|c|c|}
\hline \multirow[b]{2}{*}{$\begin{array}{c}\text { Zusammensetzung } \\
\text { in } \\
\text { Gew. } \%\end{array}$} & \multirow[b]{2}{*}{$\begin{array}{l}\mathrm{CaO} \\
\mathrm{Al}_{2} \mathrm{O}_{3} \\
\mathrm{SiO}_{2}\end{array}$} & \multirow{2}{*}{$\begin{array}{c}\begin{array}{c}\text { Komponente } \\
\mathrm{CaO} . \mathrm{Al}_{2} \mathrm{O}_{3} . \\
2 . \mathrm{SiO}_{2}\end{array} \\
20.09 \\
36.63 \\
43.28 \\
\end{array}$} & \multicolumn{4}{|c|}{ Eutektikum } & \multirow{2}{*}{\begin{tabular}{|c|}
$\begin{array}{r}\mathrm{Komponente} \\
2 \mathrm{CaO} \cdot \mathrm{Al}_{2} \mathrm{O}^{2} \\
\mathrm{SiO}_{2}\end{array}$ \\
40.83 \\
37.19 \\
21.98
\end{tabular}} \\
\hline & & & $\begin{array}{l}23.2 \\
36.8 \\
40.0\end{array}$ & $\begin{array}{l}30.2 \\
36.8 \\
33.0\end{array}$ & $\begin{array}{l}37.75 \\
37.25 \\
25.0\end{array}$ & \begin{tabular}{|l|}
35.6 \\
37.1 \\
27.3
\end{tabular} & \\
\hline Schmelztemperatur & & 1550 & 1538 & 1385 & 1586 & 1535 & 1590 \\
\hline
\end{tabular}
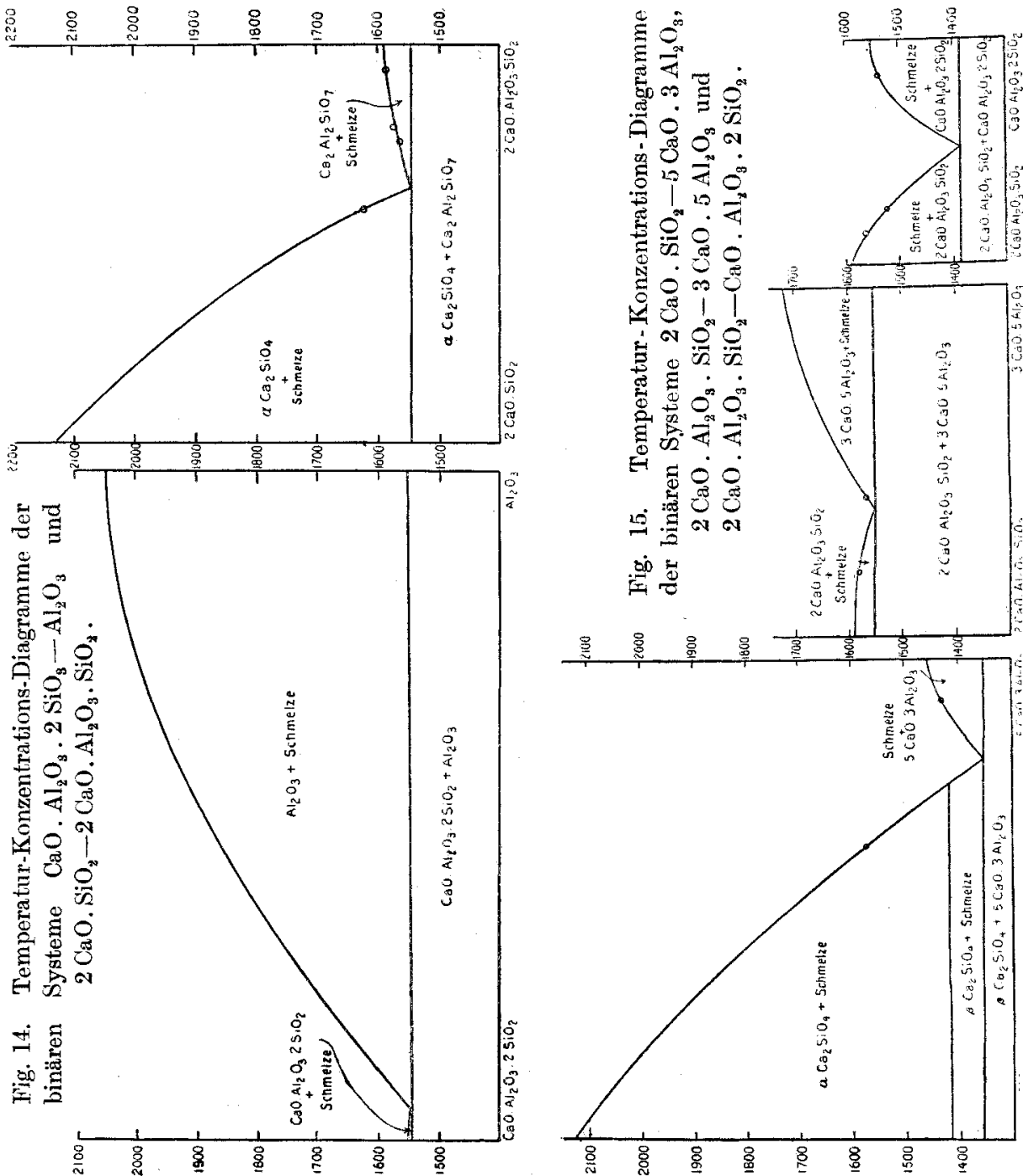
Ternäres System: Calciumoxyd-Aluminiumoxyd-Silicium-2-oxyd. 263

Tabelle 18.

Schmelztemperatur des binären Systemes

$2 \mathrm{CaO} \cdot \mathrm{Al}_{2} \mathrm{O}_{3} \cdot \mathrm{SiO}_{2}-3 \mathrm{CaO} .5 \mathrm{Al}_{2} \mathrm{O}_{3}$.

\begin{tabular}{c|c|c|c|c|c|c}
\hline \hline & & $\begin{array}{c}\text { Komponente } \\
\text { 2CaO. } \mathrm{Al}_{2} \mathrm{O}_{3} . \mathrm{SiO}_{2}\end{array}$ & \multicolumn{2}{c|}{ Eutektikum } & $\begin{array}{c}\text { Komponente } \\
\mathbf{3 C a O} .5 \mathrm{Al}_{2} \mathrm{O}_{3}\end{array}$ \\
\hline \hline Zusammensetzung & $\mathrm{CaO}$ & 40.83 & 38 & 35.0 & 34.3 & 24.78 \\
in & $\mathrm{Al}_{2} \mathrm{O}_{3}$ & 37.19 & 44 & 50.8 & 52.7 & 75.22 \\
Gew.-0\% & $\mathrm{SiO}_{2}$ & 21.98 & 18 & 14.2 & 13.0 & 0.0 \\
\hline Schmelztemperatur & & 1590 & 1583 & 1552 & 1570 & 1720
\end{tabular}

Tabelle 19.

Schmelztemperatur des binären Systemes $2 \mathrm{CaO} . \mathrm{SiO}_{2}-5 \mathrm{CaO} .3 \mathrm{Al}_{2} \mathrm{O}_{3}$.

\begin{tabular}{c|c|c|c|c|c|c}
\hline & & $\begin{array}{c}\text { Komponente } \\
2 \mathrm{CaO} . \mathrm{SiO}_{2}\end{array}$ & \multicolumn{2}{|c|}{ Eutektikum } & $\begin{array}{c}\text { Komponente } \\
5 \mathrm{CaO}_{3} .3 \mathrm{AOl}_{23}\end{array}$ \\
\hline Zusammensetzung & $\mathrm{CaO}$ & 65.0 & $\mathbf{5 4 . 5}$ & 51.3 & 49.3 & 47.78 \\
in & $\mathrm{Al}_{2} \mathrm{O}_{3}$ & 0.0 & 32.0 & 41.9 & 47.7 & 52.22 \\
Gew.-\% & $\mathrm{SiO}_{2}$ & 35.0 & 13.5 & 6.8 & 3.0 & 0.0 \\
\hline Schmelztemperatur & & 2130 & 1575 & 1350 & 1432 & 1455
\end{tabular}

Tabelle 20.

Schmelztemperatur des binären Systemes $\mathrm{CaSiO}_{3}-\mathrm{CaO} \cdot \mathrm{Al}_{2} \mathrm{O}_{3}$.

\begin{tabular}{|c|c|c|c|c|c|c|c|c|c|}
\hline \multirow[b]{2}{*}{$\begin{array}{c}\text { Zusammensetzung } \\
\text { in } \\
\text { Gew.. } \%\end{array}$} & \multirow[b]{2}{*}{$\begin{array}{l}\mathrm{CaO} \\
\mathrm{Al}_{2} \mathrm{O}_{3} \\
\mathrm{SiO}_{2}\end{array}$} & \multirow{2}{*}{$\begin{array}{c}\text { Komponente } \\
\mathrm{CaSiO}_{3} \\
48.2 \\
0.0 \\
51.8\end{array}$} & \multicolumn{7}{|c|}{ Eutektikum } \\
\hline & & & $\begin{array}{r}48.0 \\
2.0 \\
50.0\end{array}$ & $\begin{array}{r}47.0 \\
6.3 \\
46.7\end{array}$ & $\begin{array}{l}46.3 \\
10.0 \\
43.7\end{array}$ & \begin{tabular}{l|}
45.7 \\
13.2 \\
41.1
\end{tabular} & $\begin{array}{l}44.3 \\
20.0 \\
35.7\end{array}$ & $\left|\begin{array}{l}43.0 \\
26.2 \\
30.8\end{array}\right|$ & $\begin{array}{l}41 \\
36 \\
23\end{array}$ \\
\hline \multirow[t]{2}{*}{ Schmelztemperatur } & & 1540 & 1522 & 1472 & $|1405|$ & 1316 & 1477 & 1549 & 1586 \\
\hline & & \multicolumn{2}{|c|}{$\begin{array}{c}\text { Komponente } \\
2 \mathrm{CaO} \cdot \mathrm{Al}_{2} \mathrm{O}_{3} \cdot \mathrm{SiO}_{2}\end{array}$} & \multicolumn{4}{|c|}{ Eutektikum } & $\begin{array}{l}\text { Compon } \\
\mathrm{CaO} . \mathrm{A}\end{array}$ & $\begin{array}{l}\text { lente } \\
1_{2} \mathrm{O}_{3}\end{array}$ \\
\hline $\begin{array}{c}\text { Zusammensetzung } \\
\text { in } \\
\text { Gew.- } \%\end{array}$ & $\begin{array}{l}\mathrm{CaO} \\
\mathrm{Al}_{2} \mathrm{O}_{3} \\
\mathrm{SiO}_{3}\end{array}$ & $\begin{array}{l}40.83 \\
37.19 \\
21.98\end{array}$ & & $\begin{array}{l}39.2 \\
46.0 \\
14.8\end{array}$ & $\begin{array}{r}37.8 \\
52.9 \\
9.3\end{array}$ & $\begin{array}{r}36 \\
58 \\
5\end{array}$ & & $\begin{array}{r}35.44 \\
64.56 \\
0.0\end{array}$ & \\
\hline Schmelztemperatur & & 1590 & & 1572 & 1512 & 15 & 49 & 1600 & \\
\hline
\end{tabular}

b) Die gerade Linie liegt zum Teil innerhalb des Feldes der beiden Stoffe, deren Zusammensetzungen sie verbindet. Wir betrachten z. B. die Linie, die die Verbindung herstellt zwischen $C A S_{2}\left(\mathrm{CaO} \cdot \mathrm{Al}_{2} \mathrm{O}_{3} .2 \mathrm{SiO}_{2}\right)$ und $A S\left(\mathrm{Al}_{2} \mathrm{O}_{3} \cdot \mathrm{SiO}_{2}\right)$ in Fig. 12. Diese Linie schneidet nicht die Grenze (1-9) zwischen $C A S_{2}$ und $A S$, so daß kein Gemisch besteht, in dem $C A S_{2}$ und $A S$ 
im Gleichgewicht mit ihrer eigenen Lösung sind; das bedeutet, die Schmelzkurven $C A S_{2}-U$ für $C A S_{2}$ und $A S-U^{\prime}$ für $A S$ begegnen sich nicht. Diese Schmelzlinien werden getrennt durch die Linie $U-U^{\prime}$, die das Feld von $A$ kreuzt und die Schmelzkurve von $\mathrm{Al}_{2} \mathrm{O}_{3}$ in Lösungen vom $A S$ und $C A S_{2}$ darstellt; mit anderen Worten: weder $C A S_{2}$ noch $A S$ sind stabil in Lösungen von Gemischen auf der Linie $U-U^{\prime}$. Dies ist demnach ein Fall, wo zwei Verbindungen, die bei ihren Schmelzpunkten stabil sind und deren Felder eine gemeinsame Grenze haben, keine binäre Reihe bilden, weil die Linie, die die Zusammensetzungen dieser beiden Stoffe verbindet, das Feld einer anderen Verbindung kreuzt. Von diesem Fall haben wir drei Bei-

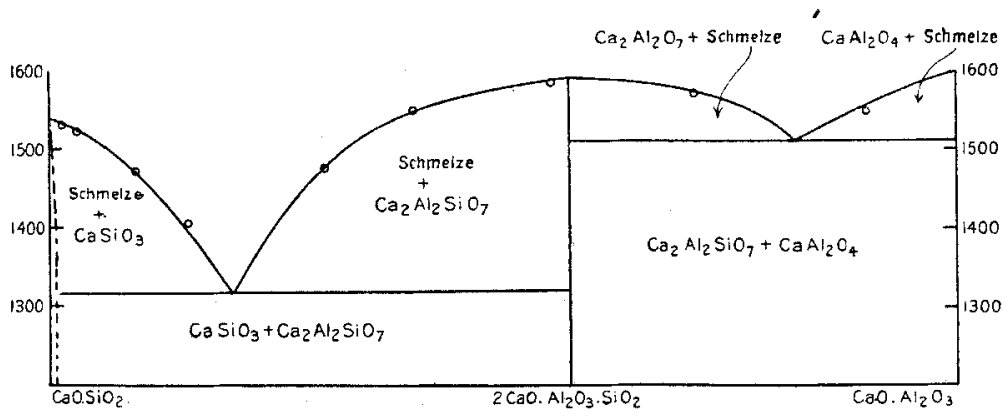

Fig. 16. Temperatur-Konzentrations-Diagramm des binären Systemes $\mathrm{CaO} . \mathrm{SiO}_{2}-\mathrm{CaO} . \mathrm{Al}_{2} \mathrm{O}_{3}$.

spiele; das eine ist bereits besprochen; ferner die Zusammensetzungslinie für $C_{2} S$ und $C A$, die das Feld für $C_{2} A S$ bei $X-X^{\prime}$ kreuzt, und die Zusammensetzungslinie für $C_{2} A S$ und $A$, die das Feld für $C_{3} A_{5}$ bei $V-V^{\prime}$ kreuzt (Fig. 12).

c) Die Zusammensetzungslinie verbindet in diesem Fall einen stabilen mit einem (bei seinem Schmelzpunkt) instabilen Stoff. Ein Beispiel ist die Linie, die die stabile Verbindung $C_{2} A S$ mit der Verbindung $C_{3} S_{2}$ vereinigt, die bei ihrem Schmelzpunkt instabil ist. Wie zu erwarten, hat die stabile Verbindung $C_{2} A S$ eine Schmelzkurve $C_{2} A S-Y$, aber die instabile Verbindung $C_{3} S_{2}$ hat keine Schmelzkurve, denn die Zusammensetzungslinie liegt nicht in ihrem Stabilitätsgebiet; dagegen kreuzt die Zusammensetzungslinie das Feld für $C_{2} S$ und die Linie $C_{3} S_{2}-Y$ ist die Schmelzkurve von $C_{2} S$ in Lösungen von $C_{3} S_{2}$ und $C_{2} A S$. Es ist noch ein anderes Beispiel für diesen Fall vorhanden: die Zusammensetzungslinie für die stabile Verbindung $C_{2} S$ und die instabile Verbindung $C_{3} A$, die die Felder für $C_{3} S$ und $C$ kreuzt. 
d) Die Zusammensetzungslinie der Stoffe $C_{3} S$ und $C_{3} A$ die beide bei ihrem Schmelzpunkt instabil sind, liegt völlig im Felde für $C$. Diese Linie ist dann die Schmelzkurve von $C$ in Lösungen von $C_{3} S$ und $C_{3} A$.

\section{Die Kristallisation im ternären System.}

Die Ergebnisse dieser Untersuchung sind bisher graphisch in Diagrammen dargestellt worden, um das Gleichgewicht zwischen den verschiedenen Phasen des Systemes von $\mathrm{CaO}-\mathrm{Al}_{2} \mathrm{O}_{3}-\mathrm{SiO}_{2}$ erkennen zu lassen. Um diese Daten bei praktischen Untersuchungen nutzbar zu machen, ist es wünschenswert $\mathrm{zu}$ wissen, welches die Zusammensetzung der festen Phasen ist, wenn eine ternäre Lösung abgekühlt wird. Die systematische Betrachtung der Theorie der Phasenausscheidung hat W. C. GeER ${ }^{1}$ in einer Arbeit durchgeführt, die den Titel trägt „Kristallisation in Systemen dreier Komponenten“. Anstatt lediglich hier diese wichtige Untersuchung anzuführen, erschien es wünschenswert, GEERs Ableitungen über Kristallisationen in Dreikomponentensystemen ziemlich ausführlich an dieser Stelle nochmals wiederzugeben, da sie die Theorie in sehr vollkommener Weise darstellen; denn wir glauben, daß die Kristallisation ternärer Lösungen selbst bei denen, die damit zu tun haben, nicht so allgemein bekannt ist und daß die Theorie von grundlegender Wichtigkeit für das Verständnis des Verhaltens eines ternären Systemes ist. Wo GEER hypothetische Verbindungen und Bedingungen benutzt hat, haben wir dafür der Wirklichkeit entnommene Daten eingesetzt, um an ihnen die Ableitungen vorzunehmen. Im übrigen ist das hier Gebotene im wesentlichen identisch mit der Abhandlung von GEER, wenn man von einigen kleinen Änderungen in der Anordnung absieht, sowie vom Wortlaut einiger Erläuterungen.

Für die Besprechung der Theorie der Kristallisation in Anwendung auf Lösungen, die nur aus den Komponenten $\mathrm{CaO}, \mathrm{Al}_{2} \mathrm{O}_{3}$ und $\mathrm{SiO}_{2}$ bestehen, werden wir Fig. 17 benutzen. In dieser Zeichnung sind dieselben Zahlen und Buchstaben für die Bezeichnung der Quintupel- und Quadrupelpunkte angewendet, wie bei den früheren Gleichgewichtsdiagrammen.

Die experimentellen Daten, die in diesem Diagramm dargestellt werden, sind derart, daß alle möglichen theoretischen Typen ternärer Kristallisation, die GEER besprochen hat, darin zu finden sind.

I Journ. Phys. Chem. 8 (1904), 257. 
Von der Besprechung werden wir die festen Lösungen ausschließen, da diese nur im Falle des $\mathrm{CaO} . \mathrm{SiO}_{2}$ auftreten und auch hier nur bis zu $2 \%$ von jeder der Verbindungen, mit denen der genannte Stoff zusammen vorkommt, nämlich $\mathrm{SiO}_{2}, 3 \mathrm{CaO} .2 \mathrm{SiO}_{2}, \mathrm{CaO} \cdot \mathrm{Al}_{2} \mathrm{O}_{3} \cdot 2 \mathrm{SiO}_{2}$ und $2 \mathrm{CaO} \cdot \mathrm{Al}_{2} \mathrm{O}_{3} \cdot \mathrm{SiO}_{2}$.

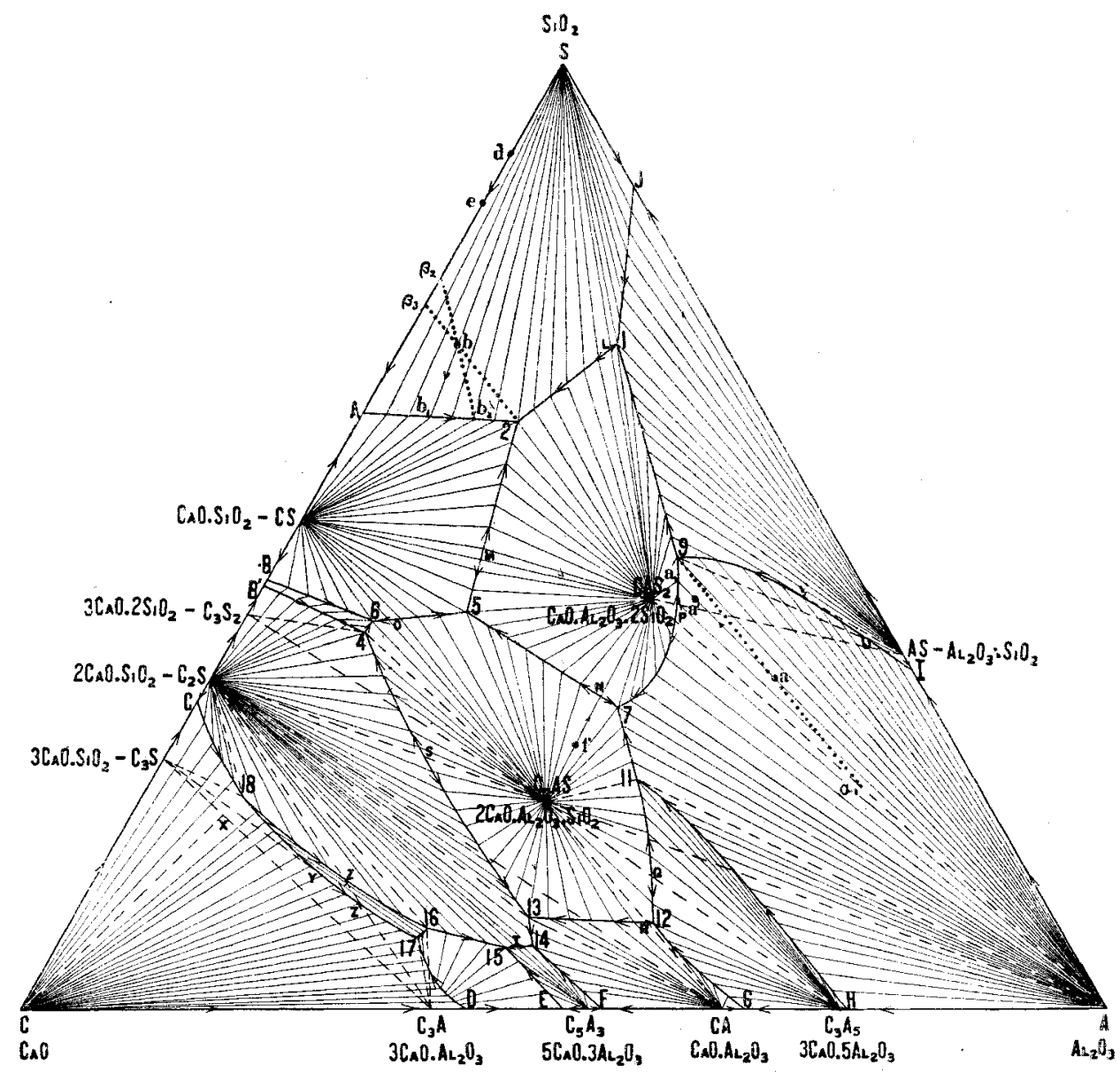

Fig. 17. Gleichgewichtsdiagramm zur Darstellung des Verlaufes der Kristallisation, wenn ternäre Lösungen von $\mathrm{CaO} ; \mathrm{Al}_{2} \mathrm{O}_{3}$ und $\mathrm{SiO}_{2}$ abgekühlt werden.

Wir wollen zunächst den einfachsten Fall der Kristallisation betrachten, der gleichzeitig bei den Lösungen aus $\mathrm{CaO}, \mathrm{Al}_{2} \mathrm{O}_{3}$ und $\mathrm{SiO}_{2}$ am häufigsten zur Beobachtung gelangt. Für diesen Zweck ist es nützlich, den Teil von Fig. 17, der eingeschlossen wird von dem Dreieck der Linien, die die Zusammensetzungen von $\mathrm{S}\left(\mathrm{SiO}_{2}\right)$, 
CS $\left(\mathrm{CaO} \cdot \mathrm{SiO}_{2}\right)$ und $\left.\mathrm{CAS}_{2}\left(\mathrm{CaO} \cdot \mathrm{Al}_{2} \mathrm{O}_{3} \cdot 2 \mathrm{SiO}\right)_{2}\right)$ verbinden, als ein besonderes und abgetrenntes ternäres System zu betrachten. Hierdurch erhalten wir ein ternäres System, in dem die drei Komponenten sich weder zu binären, noch ternären Verbindungen vereinigen. Aus Fig. 17 geht hervor, daB die Linien S-CS, S-CAS 2 und $\mathrm{CS}-\mathrm{CAS}_{2}$ drei binäre Systeme darstellen, in denen $A, L$ und $M$ die eutektischen Punkte sind, während 2 dem Quintupelpunkt entspricht. „Aus den Ableitungen von AlKemade ist bekannt, daß die Temperaturen auf den Seiten des Dreiecks gegen den binären eutektischen Punkt fallen und bei diesem Diagramm auch gegen den ternären eutektischen Punkt." 1 Die Richtung der fallenden Temperaturen ist in der Zeichnung durch Pfeile angedeutet. Fig. 17 ist natürlich eine Projektion des räumlichen Modells auf die Horizontalebene.

Wenn man eine gesättigte Lösung, deren Zusammensetzung durch irgendeinen Punkt im Dreieck S-CS-CAS ${ }_{2}$ dargestellt wird, abkühlt ${ }^{2}$, und daraus ein fester Stoff kristallisiert, so ändert sich die Konzentration der Lösung um die Menge der ausgeschiedenen Phase. Mit anderen Worten: der Unterschied zwischen der Anzahl von Kilogrammen von S, CS und $\mathrm{CAS}_{2}$ in der ursprünglichen Lösung und der Anzahl von Kilogrammen derselben Stoffe in der Restlösung ist gleich der Zahl von Kilogrammen des auskristallisierten festen Stoffes. Wenn man nun mit irgendeiner Lösung beginnt und diese abkühlt, so liegt die Zusammensetzung der zurückbleibenden Lösung nach teilweiser Kristallisation auf der geraden Linie zwischen dem Punkt, der die Zusammensetzung des kristallisierenden festen Stoffes bezeichnet und dem Punkt, der die Zusammensetzung der ursprünglichen Lösung darstellt. Eine derartige Änderung der Konzentration auf einer geraden Linie, die durch die anfängliche Lösung und den kristallisierenden festen Stoff geht, bildet ein Grundprinzip der Abscheidung von Phasen. Wenn man nach Fig. 17 eine Iüsung mit der durch $d$ dargestellten Zusammensetzung auf der Seite des Dreiecks $C S-S$ abkühlt, so kristallisiert die Komponente $S$, während die Lösung selbst ihre Konzentration in der entgegengesetzten Richtung auf der Linie $C S-S$ ändert; und bei Temperaturen unterhalb derjenigen der ursprünglichen Lösung ist ein Gleichgewicht vorhanden zwischen $S$ und einer neuen Lösung $e$.

1 GEeR, 1. c., S. 259.

2 Natürlich muß die Abkühlung so langsam erfolgen, daß fortdauernd Gleichgewicht erreicht wird. 
Mit Hilfe der Zeichnung kann man die Menge von S berechnen, die sich aus der Lösung ausgeschieden hat, und ebenso die Menge von $\mathrm{S}$, die in der Lösung $e$ noch vorhanden ist. Das Verhältnis der Linie $d-e$ zu der Linie $\mathrm{S}-e$ ist der Prozentgehalt des Gesamtgemisches der Lösung $d$, der sich als $\mathrm{S}$ beim Abkühlen von $d$ auf $e$ abgeschieden hat. Das Verhältnis von $\mathrm{S} d \mathrm{zu}$ Se ist der Prozentgehalt des Gesamtgemisches entsprechend $d$, der in der Lösung $e$ zurückbleibt. Es sei beispielsweise die Zusammensetzung von $d: 80 \mathrm{~kg} \mathrm{~S}$ und $20 \mathrm{~kg} C S$, und die von $e$ sei $70 \%$ S und $30 \%$ CS. Die Anzahl von Kilogrammen, die sich ausgeschieden haben, wird gefunden aus dem Verhältnis

$$
-\frac{d e}{\mathrm{~S} e} \cdot 100=\frac{10}{39} \cdot 100=33^{1} / \mathrm{s} \% \text {. }
$$

Demnach haben sich $331 / 3 \mathrm{~kg}$ als $\mathrm{S}$ ausgeschieden und $66^{2} / \mathrm{kg}$ von $\mathrm{S}$ und CS bleiben in der Lösung $e$ zurück. Die ursprüngliche Lösung $d$ enthielt $80 \mathrm{~kg} \mathrm{~S}$, demnach bleiben bei e $80-331 / 3=46.6 \mathrm{~kg} \mathrm{~S}$ zurück mit den ursprünglichen $20 \mathrm{~kg}$ CS. Der Prozentgehalt an S und CS in $e$ kann an der Figur kontrolliert werden aus den angeführten Zahlen. Die Lösung e enthält $46.6 \mathrm{~kg} S$ und $20.0 \mathrm{~kg} \mathrm{C}=66.6 \mathrm{~kg}$

$$
\frac{46.6}{66.6} \cdot 100=70 \% \text { S. } \quad \frac{20}{66.6} \cdot 100=30 \% \text { CS. }
$$

Wenn die Lösung $e$ weiter abgekühlt wird, so schreitet die $\mathrm{Ab}$ scheidung von $\mathrm{S}$ fort, bis der Punkt $A$ erreicht ist; da dies die eutektische Temperatur ist, so scheiden sich reines $\mathrm{S}$ und reines CS gemeinsam ab ohne weitere Konzentrationsänderungen der Lösung.

Auf dem Feld $A-2-L-S$ sind Lösungen der drei Komponenten im Gleichgewicht mit S. Wenn z. B. die durch den Punkt $b$ dargestellte Lösung abgekühlt wird, so kristallisiert $\mathrm{S}$, während die Zusammensetzung der Restlösung dargestellt wird durch einen Punkt der geraden Linie zwischen $b$ und dem Punkt, der die Zusammensetzung der kristallisierenden Phase, nämlich S, am Scheitel des Dreiecks ausdrückt. Wird die Lösung weiter gekühlt, so hat jede folgende Restlösung eine Zusammensetzung, die einem Punkt der geraden Linie zwischen $b$ und $\mathrm{S}$ entspricht, bis schließlich eine Lösung $b_{1}$ erscheint, deren Zusammensetzung auf der Grenze A-2 liegt. Die Linie $b b_{1}$ ist demnach der Ort aller Punkte, die die Zusammensetzung der aufeinanderfolgenden Lösungen darstellen, welche sich bilden, wenn die ursprüngliche Lösung $b$ unter Abscheidung von $\mathrm{S}$ als fester Phase abgekühlt wird.

Die Menge von S, das aus der Lösung $b$ (einer ternären Flüssig- 
keit) auskristallisiert, wird ebenso berechnet, wie vorher für die binäre Lösung angegeben ist. Beim Kühlen vom $b$ auf $b_{1}$ haben sich $\frac{b b_{1}}{\mathrm{~S} b_{1}} \cdot 100 \%$ der Lösung von der Zusammensetzung $b$ als $\mathrm{S}$ abgeschieden. Demnach verbleiben auch $\frac{b \mathrm{~S}}{\mathrm{~S} b_{1}} \cdot 100 \%$ der ursprünglichen Lösung $b$ als Flüssigkeit der Zusammensetzung $b_{1}$. Nach der Definition der Phasenregel für univariante Systeme ist die Lösung $b_{1}$ im Gleichgewicht mit S und CS; wenn demnach die Temperatur wieder erniedrigt wird, muB ein Gemisch von $\mathrm{S}$ und CS kristallisieren. Es ist angegeben worden, daß eine gerade Linie zwischen den Punkten der Zusammensetzung einer festen Phase und einer Lösung, aus welcher sie sich abscheidet, durch die Zusammensetzungen der Restlösungen hindurchgeht. Die Umkehrung dieses Satzes, die gleichfalls zutrifft, ist von Bancroft ${ }^{\mathbf{1}}$ als Prinzip seines Verfahrens zur Analyse fester Phasen angewandt worden. Wenn eine gegebene Lösung abgekühlt wird, muß eine gerade Linie, die die aufeinanderfolgenden Zusammensetzungen der Lösung verbindet, bei Verlängerung durch den Punkt gehen, der der mittleren Zusammensetzung des ausgeschiedenen festen Stoffes entspricht. In den bisher betrachteten Fällen ist diese mittlere Zusammensetzung nichts anderes als die einer reinen Verbindung. Wenn jedoch zwei Phasen sich nebeneinander abscheiden, wie die Komponenten $\mathrm{S}$ und $\mathrm{SC}$ auf der Grenze $A-2$, so wird die mittlere Zusammensetzung des sich beim Abkühlen ausscheidenden festen Stoffes - vorausgesetzt, daß kein fester Stoff während dieses Vorganges entfernt wird - gefunden werden als ein Punkt auf der Linie, die die Zusammensetzung der konstituierenden Phasen verbindet. In dem erwähnten Fall ist diese Linie die Seite des Dreiecks S-CS. Eine solche Linie kann sowohI die reinen Komponenten verbinden wie eine Komponente und eine Verbindung oder auch zwei Verbindungen.

Da die mittlere.Zusammensetzung des gesamten, bei bestimmter Temperatur abgeschiedenen festen Stoffes auf der Linie liegt, die die Zusammensetzungen der konstituierenden Phasen verbindet, sowie auf den Verlängerungen der Linie durch die Zusammensetzungen der ursprünglichen und der zurückbleibenden Lösungen, so folgt, daß sie im Schnittpunkt beider Linien liegt. Demnach wird die Verbindungslinie vom $b$ und $b_{2}$ (in Fig. 17) bei Verlängerung auf $\beta_{2}$ auf der Linie S-CS treffen, welches die mittlere Zusammensetzung

1 Journ. Phys. Chem. 6 (1902), 178. 
des festen Stoffes angibt, der sich bei Abkühlung der Lösung $b$ von der Temperatur der Isotherme durch diesen Punkt auf die Isotherme durch $b_{2}$ abscheidet. In ähnlicher Weise wird beim Abkühlen von $\tilde{b}$ bis 2 die mittlere Zusammensetzung der festen Phase dargestellt durch $\beta_{3}$. Wenn die Temperatur des Quintupelpunktes 2 erreicht ist, tritt $\mathrm{CAS}_{2}$ als Phase in dem festen Stoff auf; aber nach der Definition des eutektischen Punktes haben Lösung und Kristalle dieselbe Zusammensetzung, so daß keine weitere Änderung der Konzentration eintritt.

Bei jeder zwischenliegenden Temperatur ist dieselbe Beweisführung anwendbar. Wir betrachten z. B. eine Lösung der Zusammensetzung $b_{1}$, die auf $b_{2}$ abgekühlt wird. Da die beiden Lösungen auf der Grenzkurve liegen, so kristallisieren S und CS nebeneinander aus. Die Verlängerung durch $b_{1}$ und $b_{2}$ bis zur Seite des Dreiecks S-CS liefert $A$, und dieser Punkt zeigt die mittlere Zusammensetzung - ausgedrückt durch S und. CS - des festen Stoffes, der sich während der Abkühlung von $b_{1}$ bis $b_{2}$ abgeschieden hat.

Die Menge des erhaltenen festen Stoffes zwischen den Temperaturen von $b$ und $b_{2}$ wird wie oben gegeben durch das Verhältnis $\frac{b b_{z}}{\beta_{2} b_{2}}$. Der Bruchteil der ursprünglichen Lösung $b$, der bei $b_{2}$ in Lösung bleibt, ist $\frac{\beta_{q} b}{\beta_{3} b_{2}}$.

Aus dem Grundgesetz der Abscheidung fester Stoffe sowie aus geometrischen Überlegungen ergibt sich, daß

$$
\frac{b b_{2}}{\beta_{2} b_{2}}+\frac{\beta_{2} b}{\beta_{2} b_{2}}=1
$$

Ähnliches gilt für andere Paare von Temperaturen (oder Zusammensetzungen) in dem Gebiet.

„Der Ausdruck Kristallisationskurve wird benutzt werden, um den Ort der Punkte zu bezeichnen, die die Zusammensetzung der Lösungen darstellen, welche aus einer gegebenen Lösung entstehen, wenn diese von einer gegebenen Temperatur bis zum Quintupelpunkt erkaltet, bei dem sie fest wird, und zwar unter der Annahme, daß während der Abkühlung keine Phase entfernt wird."1 Die besprochene Kristallisationskurve beginnt bei $b$, ist bis $b_{1}$ eine gerade Linie, wendet sich hier scharf um und folgt dann der Grenzlinie bis 2. Eine von irgendeinem Punkt der Kristallisationskurve durch die ursprüngliche Lösung gezogene gerade Linie trifft in ihrer

1 GEER, I. c., S. 263. 
Verlängerung den Punkt, der die mittlere Zusammensetzung der festen Phasen darstellt, welche sich bis zu diesem Augenblick beim Abkühlen ausgeschieden haben. So geht die Verlängerung der Linie $b_{1}-b$ nach $\mathrm{S}$; die Verlängerung vom $b_{2} b$ trifft $\beta_{2}$ usw. Wenn der Punkt 2 zuerst erreicht wird, hat die feste Phase die Zusammensetzung $\beta_{3}$. Bei weiterer Verfestigung der Lösung ändert sich die mittlere Zusammensetzung der festen Phase von $\beta_{3}$ nach $b$ und erreicht diesen letzteren Punkt, wenn der letzte Tropfen der Flüssigkeit verschwindet.

Aus dieser Untersuchung der Kristallisation einer Lösung $b$ lassen sich gewisse Verallgemëinerungen ziehen.

1. Die Kristallisationskurve für irgendeine Lösung, deren Zusammensetzung in einem Feld liegt, wird auf einer bestimmten geraden Linie bis zur Grenzkurve fortschreiten.

2. Die mittlere Zusammensetzung des kristallisierenden festen Stoffes oder der zurückbleibenden Flüssigkeit an irgendeinem Punkt der Kristallisationskurve oder die Menge des auskristallisierten festen Stoffes läßt sich geometrisch aus dem Gleichgewichtsdiagramm ableiten.

Wir werden jetzt die Natur der Kristallisationskurve für irgendeine Lösung von $\mathrm{CaO}, \mathrm{Al}_{2} \mathrm{O}_{3}$ und $\mathrm{SiO}_{2}$ bis zur Grenzkurve betrachten. Wie bereits dargelegt, bildet die Kristallisationskurve für irgendeine Lösung zunächst eine gerade Linie, bis diese eine Grenzkurve trifft, and der Ursprung der geraden Linie ist derjenige Punkt, der die Zusammensetzung der primären Phase der Lösung darstellt. Um den Verlauf der Kristallisationkurven in jedem Feld zu zeigen, sind gerade Linien gezogen worden, die von dem Punkt ausstrahlen, der die Zusammensetzung der primären Phase für jedes Feld darstellt; diese Linien enden auf den Grenzkurven eines jeden dieser Felder. So liegt im Felde $\mathrm{SiO}_{2}-A-2-1-J \mathrm{SiO}_{2}$, die primäre Phase, und die von $\mathrm{SiO}_{2}$ nach den Kurven $A-2,2-1$ und $1-J$ ausstrahlenden Linien zeigen die Richtung der Kristallisationskurven bis zu den Grenzlinien für alle Lösungen, deren Zusammensetzungen innerhalb dieses Feldes liegen. In jedem Feld, wo die primäre Phase eine bei ihrem Schmelzpunkt stabile Verbindung ist, treffen sich diese gerade Linien tatsächlich bei dem Punkt, der die Zusammensetzung der primären Phase darstellt; wenn jedoch die Verbindung bei ihrem Schmelzpunkt nicht stabil ist, so treffen sich diese Linien nicht bei dem Punkt, der die Zusammensetzung der primären Phase darstellt, sondern die Richtung dieser Linien wird erhalten, wenn man diesen 
Punkt als imaginären Ausgangspunkt annimmt. So ist $3 \mathrm{CaO} .2 \mathrm{SiO}_{2}$ bei seinem Schmelzpunkt instabil, und seine Zusammensetzung liegt demnach außerhalb des Feldes; die Richtung der Kristallisationskurve irgendeiner Lösung im Felde $B-B^{\prime}-4-6$ wird erhalten, wenn man gerade Linien von dem Punkte $3 \mathrm{CaO} .2 \mathrm{SiO}_{2}$ durch dies Feld zieht; wie sich aus der Zeichnung ergibt, schneiden sich diese Linien nicht. In derselben Weise sind die Linien im Felde 16-17-18 der instabilen Verbindung $3 \mathrm{CaO} . \mathrm{SiO}_{2}$ und im Felde $D-17-16-15-E$ der instabilen Verbindung $3 \mathrm{CaO} \cdot \mathrm{Al}_{2} \mathrm{O}_{3}$ gezogen worden.

Es ist demnach augenscheinlich, daß beim Abkühlen irgendeiner Lösung von $\mathrm{CaO}, \mathrm{Al}_{2} \mathrm{O}_{3}, \mathrm{SiO}_{2}$ ihre Kristallisationskurve einer bestimmten geraden Linie bis zur. Grenzkurve folgt, und daß die Richtung fallender Temperatur auf einer solchen Linie vom Ursprung (der Zusammensetzung der Verbindung) gegen die Grenze erfolgt; wie in Fig. 17 dargestellt ist, folgt nach Erreichung der Grenzkurve die Kristallisationskurve der Richtung fallender Temperatur, wie die Pfeile anzeigen.

Wenn die Kristallisationskurve der Lösung $b$ die. Grenzkurve erreicht, so folgt sie dieser, und es kristallisieren dann zwei Phasen, bis der ternäre eutektische Punkt erreicht ist, worauf drei Phasen kristallisieren und die Lösung vollständig fest wird. Alle Lösungen von $\mathrm{CaO}, \mathrm{Al}_{2} \mathrm{O}_{3}, \mathrm{SiO}_{2}$, deren Kristallisationskurven auf eine Grenze zulaufen, die direkt zu einem eutektischen Punkt führt, besitzen diesen Typus der Kristallisationskurve. Die ternären eutektischen Punkte, bei denen diese Art von Kristallisationskurven ihr Ende finden, sind im Diagramm durch die Zahlen 1, 2, 5, 6, 7, 12, 14, 15 bezeichnet. Es folgt demnach, daß die Kristallisation irgendeiner Lösung, deren Kristallisationskurve eine Grenzkurve schneidet, die mit fallender Temperatur (das heißt in Richtung der Pfeile) auf einen dieser eutektischen Punkte zuläuft, kontinuierlich erfolgt, bis die Lösung völlig fest geworden ist.

Einige Paare von festen Phasen verhalten sich als binäres System; wenn eine Lösung einer Zusammensetzung, die genau auf der Verbindungslinie eines solchen Paares liegt, z. B. $f$ auf der Verbindungslinie von $2 \mathrm{CaO} \cdot \mathrm{Al}_{2} \mathrm{O}_{3} \cdot \mathrm{SiO}_{2}$ und $\mathrm{CaO} \cdot \mathrm{Al}_{2} \mathrm{O}_{3} \cdot 2 \mathrm{SiO}$, abgekühlt wird, so kristallisiert $2 \mathrm{CaO} \cdot \mathrm{Al}_{2} \mathrm{O}_{3} \cdot \mathrm{SiO}_{2}$ und die Kristallisationskurve geht bis zum Quadrupelpunkt $N$, wo die Lösung vollständig fest wird, indem $\mathrm{C}_{2} \mathrm{AS}$ und $\mathrm{CAS}_{2}$ gemeinschaftlich kristallisieren. Neun solche binäre Eutektika sind in diesem ternären System vorhanden, nämlich $L, M, N, O, P, Q, R, S$ und $T$. Alle Kristalli- 
sationskurven, die eine Grenze bei einem dieser Punkte treffen, finden auch in diesem Punkt ihr Ende.

Bei den ternären Kristallisationskurven, die bisher betrachtet wurden, verlief die Kristallisation vom Beginn bis zum Ende der Kurve kontinuierlich, und der Endpunkt war ein eutektischer Punkt, in dem drei feste Phasen nebeneinander kristallisierten; auf solchen Kristallisationskurven kann die Zusammensetzung der flüssigen Phase in jedem Augenblick hergestellt werden aus den drei festen Phasen, die nebeneinander im eutektischen Punkt kristallisieren. Mit anderen Worten: eine solche Kristallisationskurve liegt völlig innerhalb des Dreiecks, das entsteht, wenn man die Zusammensetzungen der drei festen Phasen verbindet, die beim Quintupelpunkt vorhanden sind; denn die Fläche in diesem Dreieck stellt alle möglichen Konzentrationen dieser drei festen Phasen dar. So beginnt die Kristallisationskurve der Lösung $b$ natürlich bei $b$ und endet im eutektischen Punkt 2; ihr Verlauf liegt ganz in dem Dreieck S-CS-CAS 2 , das alle möglichen Konzentrationen dieser drei Komponenten darstellt. Demnach bedeuten alle Punkte dieser Fläche Lösungen von S, CS, $\mathrm{CAS}_{2}$.

Wir kommen jetzt zur Betrachtung der Kristallisationskurven, die zum Teil außerhalb des Dreiecks liegen, das gebildet wird aus den Linien, die die Zusammensetzungen der drei im Quintupelpunkt vorhandenen festen Phasen verbinden; dieser Punkt ist in einem solchen Falle nicht eutektisch, bildet aber das Ende der Kristallisationskurven. Als Beispiel wollen wir die Lösung $a$ im Dreieck $\mathrm{CAS}_{2}-\mathrm{AS}-\mathrm{A}$ betrachten (Fig. 17). Die Kristallisationskurve beginnt bei $a$ und ist bis $a_{1}$ eine gerade Linie; sie folgt dann der Grenze P-9 bis Punkt 9, wo sie ihr Ende findet. Vom $a$ bis $a_{1}$ kristallisiert A und vom $a_{1}$ bis 9 kristallisiert neben $\mathrm{A}$ auch $\mathrm{CAS}_{2}$. Die Zusammensetzung der Lösung ist gegeben durch die Punkte auf der Kristallisationskurve $a-a_{1}-9$; die Zusammensetzung des kristallisierenden festen Stoffes erhält man, wenn man die gerade Linie durch $a$ und die Punkte von $a_{1}-9$ bis zum Schnittpunkt der Zusammensetzungslinie $\mathrm{CAS}_{2}-\mathrm{A}$ verlängert. Der schließlich vorhandene feste Stoff wird natürlich dieselbe Zusammensetzung haben wie die ursprüngliche Lösung $a$, wenn nichts Festes im Verlauf der Kristallisation entfernt worden ist. Man sieht, daß Punkt 9 außerhalb des Dreiecks $\mathrm{CAS}_{2}-\mathrm{AS}-\mathrm{A}$ liegt; das heißt, Punkt 9, der die Zusammensetzung der Lösung darstellt, wenn die Kristallisationskurve 9 erreicht, hat derartige Zusammensetzung, daß sie nicht durch $\mathrm{CAS}_{2}$, AS und A 
allein ausgedrückt werden kann. Wir wissen aber, daß die Lösung $a$ bei der Temperatur von 9 fest wird, und daß die drei vorhandenen festen Phasen $\mathrm{CAS}_{2}$, AS und A sind. Wenn dies der Fall ist, so bleibt nur ein Weg übrig, auf dem dies Ergebnis erreicht werden kann: daß nämlich ein Teil vom auskristallisierten $\mathrm{Al}_{2} \mathrm{O}_{3}$ sich mit $\mathrm{SiO}_{2}$ in Lösung unter Bildung von $\mathrm{AS}\left(\mathrm{Al}_{2} \mathrm{O}_{3}\right.$. $\left.\mathrm{SiO}_{2}\right)$ vereinigt, und das findet in der Tat statt. Sobald die Kristallisationskurve den Punkt 9 erreicht, vereinigt sich $\mathrm{Al}_{2} \mathrm{O}_{3}$ mit $\mathrm{SiO}_{2}$ in Lösung zu $\mathrm{Al}_{2} \mathrm{O}_{3}$. $\mathrm{SiO}_{2}$ und $\mathrm{CaO} \cdot \mathrm{Al}_{2} \mathrm{O}_{3} \cdot 2 \mathrm{SiO}_{2}$ kristallisiert weiter. Dies geht so fort, bis entweder $\mathrm{SiO}_{2}$ aus der Lösung oder $\mathrm{Al}_{2} \mathrm{O}_{3}$ aus dem festen Stoff verschwunden ist. In dem besprochenen Fall (bei Lösung a) wird $\mathrm{SiO}_{2}$ zuerst verschwinden, und da die Flüssigkeit bei 9 fest wird, so ändert sich die Zusammensetzung der festen Phase von $\alpha_{1}$ bis $a$ und erreicht diesen letzten Punkt, wenn der letzte Tropfen Flüssigkeit verschwindet.

Wenn wir jedoch von einer Lösung $a^{\prime}$ ausgehen, deren Zusammensetzung in dem Dreieck liegt, das alle möglichen Konzentrationen von $\mathrm{S}, \mathrm{AS}$ und $\mathrm{CAS}_{2}$ darstellt, dann wird $\mathrm{Al}_{2} \mathrm{O}_{3}$, wenn die Kristallisationskurve $a^{\prime}-a_{1}-9$ den Punkt 9 erreicht, aus den festen Phasen verschwinden, bevor alles $\mathrm{SiO}_{2}$ aus der Lösung aufgebraucht ist, und die Kristallisationskurve folgt der Grenze 9-1, indem AS und $\mathrm{CAS}_{2}$ nebeneinander kristallisieren. Bei Punkt 1 endet die Kristallisation und die Flüssigkeit wird fest, indem AS, S und $\mathrm{CAS}_{2}$ nebeneinander kristallisieren, und die Gesamtzusammensetzung der festen Phasen ändert sich wieder bis $a^{\prime}$.

Es sind verschiedene Teile des Diagrammes vorhanden mit solchen Konzentrationen, daß ihre Lösungen diesen allgemeinen Typus der Kristallisationskurve besitzen. So haben alle Flüssigkeiten, die durch Punkte innerhalb der Fläche $\mathrm{C}_{2} \mathrm{AS}-11-\mathrm{A}-\mathrm{C}_{3} \mathrm{~A}_{5}$ dargestellt werden, Kristallisationskurven, auf denen die Kristallisation kontinuierlich erfolgt, bis der Quintupelpunkt 11 erreicht ist. Einige dieser Kurven werden bei diesem Punkt enden, andere werden der Grenze 11-7 folgen und im eutektischen Punkt 7 endigen. Folgt man den Ausführungen über die Kristallisationskurven für die Lösungen $a$ und $a_{1}$, so ist es augenscheinlich, daß die Lösungen im Dreieck $\mathrm{C}_{2} \mathrm{AS}-\mathrm{A}-\mathrm{C}_{3} \mathrm{~A}_{5}$ Kristallisationskurven haben, die im Quintupelpunkt 11 endigen, und daß diese Lösungen im Dreieck $\mathrm{C}_{2} \mathrm{AS}-11-\mathrm{A}$ durch den Punkt 11 gehen, wo $\mathrm{C}_{3} \mathrm{~A}_{5}$ völlig verschwindet, und sich entlang der Grenze 11-7 bis Punkt 7 fortsetzen, wo die Kristallisationskurve endet, indem $\mathrm{A}, \mathrm{C}_{2} \mathrm{AS}$ und $\mathrm{CAS}_{2}$ zusammen kristallisieren. 
In derselben Weise kann gezeigt werden, daß die Kristallisationskurven für alle Lösungen der Fläche $\mathrm{C}_{2} \mathrm{~S}-\mathrm{C}_{2} \mathrm{AS}-\mathrm{CA}-14$ zuerst bis zum Quintupelpunkt 13 verlaufen. Für die Flüssigkeiten im Dreieck $\mathrm{C}_{2} \mathrm{~S}-\mathrm{C}_{2} \mathrm{AS}-\mathrm{CA}$ enden die Kristallisationskurven bei 13 und für die Flüssigkeiten des Dreiecks $\mathrm{C}_{2} \mathrm{~S}-\mathrm{CA}-14$ gehen die Kristallisationskurven durch 13 hindurch und enden im eutektischen Punkt 14.

Bei allen bisher untersuchten Kristallisationskurven scheiden sich auf allen Punkten der Grenzkurven zwei Phasen nebeneinander ab; mit anderen Worten, die Zusammensetzung der festen Phase auf einer Grenzkurve konnte dargestellt werden durch die beiden sich abscheidenden festen Phasen. Dies war deswegen der Fall, weil die Verbindungslinie zweier beliebiger Punkte auf der Grenzkurve bei Verlängerung die gerade Linie schnitt, die die Zusammensetzungen der beiden sich abscheidenden Phasen verband. Es kommen jedoch Fälle vor, bei denen die Verlängerung einer solchen Linie nicht die Zusammensetzungslinie der sich abscheidenden Phasen selbst schneidet, sondern die Verlängerung dieser Linie.

Wir betrachten z. B. die Lösung $r$, deren Zusammensetzung im Dreieck CS $-\mathrm{C}_{3} \mathrm{~S}_{2}-\mathrm{C}_{2} \mathrm{AS}$ liegt. $\mathrm{C}_{2} \mathrm{~S}$ scheidet sich ab, bis die Kristallisationskurve die Grenzkurve $B^{\prime}-4$ bei $r_{1}$ erreicht hat (Fig. 18). (Fig. 18 ist ein Teil von Fig. 17 in vergrößertem Maßstab.) Von diesem Punkt wird die Grenzkurve bis 4 verfolgt; wobei der feste Stoff aus $\mathrm{C}_{2} \mathrm{~S}$ und $\mathrm{C}_{3} \mathrm{~S}_{2}$ besteht. $\mathrm{Da}$ die Zusammensetzung der Flüssigkeit auf der Grenzkurve $B^{\prime}-4$ von $r_{1}$ bis 4 sich ändert, so erkennt man aus der Figur, daß die mittlere Zusammensetzung des gesamten ausgeschiedenen festen Stoffes von $\mathrm{C}_{2} \mathrm{~S}$ bis $\sigma$ sich ändert. Die mittlere Zusammensetzung desjenigen festen Stoffes, der sich während des Abkühlens von $r_{1}$ bis 4 abgeschieden hat, wird gefunden durch Verlängerung der Linie, die $r_{1}$ und 4 verbindet; es ist $B^{\prime}$. $B^{\prime}$ liegt nun jedoch außerhalb der Linie, die die Phasen verbindet, welche mit der Flüssigkeit im Gleichgewicht sind, nämlich $\mathrm{C}_{2} \mathrm{~S}, \mathrm{C}_{3} \mathrm{~S}_{2}$. Demnach kann durch keinerlei Vereinigung von $\mathrm{C}_{2} \mathrm{~S}$ und $\mathrm{C}_{3} \mathrm{~S}_{2}$ ein Gemisch hergestellt werden, das die Konzentration $B^{\prime}$ besitzt. Es können sich demnach auch nicht $\mathrm{C}_{2} \mathrm{~S}$ und $\mathrm{C}_{3} \mathrm{~S}_{2}$ beide während der Abkühlung von $r_{1}$ bis 4 abgeschieden haben, sondern in Wirklichkeit hat sich $\mathrm{C}_{3} \mathrm{~S}_{2}$ allein abgeschieden, während $\mathrm{C}_{2} \mathrm{~S}$ verschwunden ist. Wenn mehr $\mathrm{C}_{2} \mathrm{~S}$ aus dem festen Stoff verschwindet, als sich mit $C S$ in Lösung unter Bildung von $\mathrm{C}_{3} \mathrm{~S}_{2}$ vereinigt, dann muB $\mathrm{C}_{2} \mathrm{~S}$ sich in Wirklichkeit auflösen. Aus der Lösung $r$ wird dann $\mathrm{C}_{2} \mathrm{~S}$ kristallisieren, bis die Kristallisationskurve die Grenze $B^{\prime}-4$ bei $r_{1}$ 
schneidet. Von $r_{1}$ bis 4 wird $\mathrm{C}_{2} \mathrm{~S}$ völlig oder zum Teil verschwinden und $\mathrm{C}_{3} \mathrm{~S}_{2}$ wird kristallisieren. Wenn 4 erreicht wird, so ist die Konzentration des festen Stoffes $\sigma$. Die Temperatur wird jetzt konstant bleiben, während $\mathrm{C}_{2} \mathrm{AS}$ neben $\mathrm{C}_{3} \mathrm{~S}_{2}$ kristallisiert; das feste $\mathrm{C}_{2} \mathrm{~S}$ wird unter Bildung von $\mathrm{C}_{3} \mathrm{~S}_{2}$ aufgebraucht, bevor das CS in der Flüssigkeit verschwindet, da die Konzentration von CS in der Flüssigkeit hoch ist. Demnach wird die Kristallisation entlang der Grenze 4-6

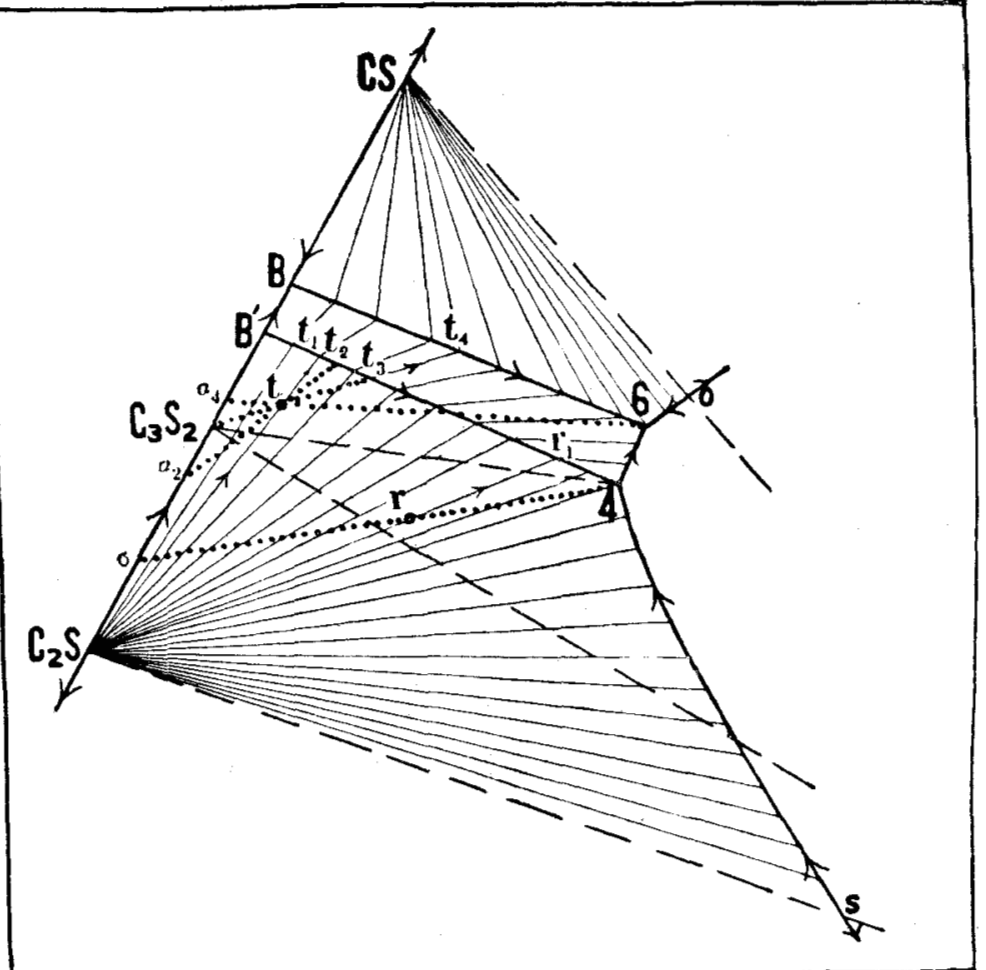

Fig. 18. Vergrößerter Teil von Fig. 17 zur Darstellung der ,Wiederauflösung““

fortschreiten, $\mathrm{C}_{2} \mathrm{AS}$ und $\mathrm{C}_{3} \mathrm{~S}_{2}$ werden sich abscheiden, bei 6 wird CS neben $\mathrm{C}_{2} \mathrm{AS}$ und $\mathrm{C}_{3} \mathrm{~S}_{2}$ kristallisieren, und die Kristallisationskurve endet in diesem Punkt.

In dem soeben besprochenen Fall verschwand eine auskristallisierte Phase zum Teil wieder, während die Kristallisation auf der Grenzkurve fortschritt. Es gibt auch Lösungstypen, bei denen eine auskristallisierte Phase völlig verschwindet, wenn die Kristallisationskurve einer Grenzkurve folgt. Eine solche Lösung ist die durch Punkt $t$ (Fig. 18) im Dreieck $\mathrm{CS}-\mathrm{C}_{3} \mathrm{~S}_{2}-\mathrm{C}_{2} \mathrm{AS}$ dargestellt. Da die 
Lösung $t$ im Felde für $\mathrm{C}_{2} \mathrm{~S}$ liegt, so wird $\mathrm{C}_{2} \mathrm{~S}$ der erste feste Stoff sein, der auftritt, wenn die Lösung abgekühlt wird. Wie in den früheren Fällen ändert sich die Zusammensetzung der Lösung auf einer geraden Linie und schneidet die Grenze $B^{\prime}-4$ bei $t_{1}$. Diese Lösung ist gesättigt sowohl in bezug auf $\mathrm{C}_{2} \mathrm{~S}$ wie auf $\mathrm{C}_{3} \mathrm{~S}_{2}$, und wenn demnach die $t_{2}$ entsprechende Temperatur erreicht ist, so besteht der feste Stoff aus $\mathrm{C}_{2} \mathrm{~S}$ und $\mathrm{C}_{3} \mathrm{~S}_{2}$ und seine mittlere Zusammensetzung ist $\alpha_{2}$. Bei weiterem Abkühlen wird ein Punkt $t_{3}$ erreicht, bei dem die Zusammensetzung des festen Stoffes der Zusammensetzung der Verbindung $\mathrm{C}_{3} \mathrm{~S}_{2}$ entspricht. Während des Abkühlens der Lösung $t$ durch die Punkte $t_{1}, t_{2}, t_{3}$ zeigt eine Verlängerung bis zur Linie, welche die Zusammensetzungen der sich abscheidenden reinen Phasen verbindet, daß der Prozentgehalt an $\mathrm{C}_{2} \mathrm{~S}$ in der Kristallmasse stetig abgenommen hat. Da die feste Phase, die ursprünglich aus $\mathrm{C}_{2} \mathrm{~S}$ bestand, sich kontinuierlich bis zur Zusammensetzung der Verbindung $\mathrm{C}_{3} \mathrm{~S}_{2}$ ändert, während $\mathrm{C}_{3} \mathrm{~S}_{2}$ und $\mathrm{C}_{2} \mathrm{~S}$ sich nebeneinander abscheiden, so folgt, da $\mathrm{B}$ diese Änderung bedeutet, daß reines $\mathrm{C}_{2} \mathrm{~S}$ schneller verschwindet, als es sich abscheidet; mit anderen Worten: die Verbindung $\mathrm{C}_{2} \mathrm{~S}$, die sich beim Abkühlen entlang der Kurve von $t$ bis $t_{1}$ ausgeschieden hat, ist verschwunden beim Abkühlen von $t_{1}$ bis $t_{3}$, während die Verbindung $\mathrm{C}_{3} \mathrm{~S}_{2}$ auskristallisiert ist. Mit der Lösung $t_{3}$ steht demnach im Gleichgewicht dann nur die Verbindung $\mathrm{C}_{3} \mathrm{~S}_{2}$.

Wenn die Lösung $t_{3}$ abgekühlt wird, so scheint wieder nur einer von den beiden Wegen der Kristallisationskurven eingeschlagen zu werden. Sie könnte der Grenzkurve folgen bis zum Quintupelpunkt 4, wie in den früheren Fällen, wobei dann die mittlere Zusammensetzung des festen Stoffes im Gleichgewicht mit der Lösung gegeben w ürde durch Punkte auf $\mathrm{C}_{2} \mathrm{~S}-\mathrm{CS}$, aber auf der von $\mathrm{C}_{2} \mathrm{~S}$ abgekehrten Seite von $\mathrm{C}_{3} \mathrm{~S}_{2}$. Ein solcher Verlauf ist jedoch unmöglich, weil kein Gemisch von $\mathrm{C}_{2} \mathrm{~S}$ and $\mathrm{C}_{3} \mathrm{~S}_{2}$ besteht, das durch irgendeine mögliche Verbindung auf einen geringeren Gehalt an $\mathrm{C}_{2} \mathrm{~S}$ gebracht werden kann, als für die Zusammensetzung von $\mathrm{C}_{3} \mathrm{~S}_{2}$ erforderlich ist. Demnach kann die Kristallisationskurve nicht der Grenzkurve $B^{\prime}-4$ bis zum Quintupelpunkt 4 folgen. Die Richtigkeit dieses Schlusses ergibt sich auch aus der Betrachtung der folgenden Tatsachen: Da $\mathrm{C}_{2} \mathrm{~S}$ während des Abkühlens von $t_{1}$ bis $t_{3}$ verschwindet und da $\mathrm{C}_{2} \mathrm{~S}$ nicht mehr für eine solche Änderung vorhanden ist und da $\mathrm{C}_{\mathbf{3}} \mathrm{S}_{\mathbf{2}}$ bei der fraglichen Temperatur stabil ist, so kann die Kristallisationskurve nicht der Grenzkurve über $t_{3}$ hinaus folgen. Infolgedessen 
schlägt die Kristallisationskurve beim weiteren Abkühlen den einzigen anderen möglichen Weg ein. $\mathrm{C}_{3} \mathrm{~S}_{2}$ wird weiter abgeschieden, die Kristallisationskurve verläßt die Grenze, kreuzt das Feld $B^{\prime}-B-$ 4-6, in dem $\mathrm{C}_{3} \mathrm{~S}_{2}$ feste Phase ist, und trifft auf die Grenze $B-6$ bei $t_{4}$. In Übereinstimmung mit der Gleichgewichtslehre ist die Lösung $t_{4}$ sowohl an CS als auch an $\mathrm{C}_{3} \mathrm{~S}_{2}$ gesättigt. Beim Abkühlen der Flüssigkeit geht demnach die Kristallisationskurve auf der Grenze $B-6$ fort, indem sich $\mathrm{CS}$ und $\mathrm{C}_{3} \mathrm{~S}_{2}$ abscheidet. Die mittlere Zusammensetzung des festen Stoffes bei $t_{4}$ ist $\mathrm{C}_{3} \mathrm{~S}_{2}$ und bei 6 - dem Ende der Kurve ist sie $\alpha_{4}$.

Bei der Abkühlung der Lösung $t$ sind demnach nacheinander aufgetreten: reines $\mathrm{C}_{2} \mathrm{~S}, \mathrm{C}_{2} \mathrm{~S}$ und $\mathrm{C}_{3} \mathrm{~S}_{2}$; reines $\mathrm{C}_{3} \mathrm{~S}_{2}, \mathrm{C}_{3} \mathrm{~S}_{2}$ und $\mathrm{CS}$ und schlieBlich $\mathrm{C}_{3} \mathrm{~S}_{2}$, CS und $\mathrm{C}_{2} \mathrm{AS}$. Man erkennt demnach, daß wenn ein ternäres System mit einer bei ihrem Schmelzpunkt instabilen Verbindung auftritt (in diesem Falle $\mathrm{C}_{3} \mathrm{~S}_{2}$ ), Lösungen gefunden werden können, bei denen die Kristallisationskurven nicht der ersten geschnittenen Grenzkurve bis zum Quintupelpunkt folgen, sondern daß sie in das Feld für die Verbindung eintreten, und einer anderen Grenze bis zu einem anderen Quintupelpunkt folgen können. Aus einer Betrachtung von Fig. 18 ergibt sich, daß die Kristallisationskurve die Grenze nur dann verlassen kann, wenn ein Punkt errejcht ist, von dem die Linie, die durch den Punkt gezogen ist, der der Zusammensetzung der ursprünglichen Lösung entspricht, auch durch die Zusammensetzung der fraglichen Verbindung geht. Lösungen, für die diese Bedingung zutrifft, liegen in einem begrenzten Feld. Bei einer Betrachtung von Fig. 18 erkennt man, daß solche Lösungen soweit $\mathrm{C}_{3} \mathrm{~S}_{2}$ in Betracht kommt, nur in den Grenzen des Dreiecks $\mathrm{C}_{3} \mathrm{~S}_{2}-B^{\prime}-4$ auftreten können. Diese Überlegungen sind augenscheinlich auf binäre und ternäre Verbindungen in gleicher Weise anwend bar.

Bei der Behandlung der Kristallisationskurven, die zu einer Grenze gelangen, auf der eine feste Phase gänzlich oder zum Teil verschwindet, haben wir den fall betrachtet, bei dem die Grenzkurve, welche das Feld einer bei ihrem Schmelzpunkt stabilen Verbindung von dem Feld einer bei ihrem Schmelzpunkt instabilen Verbindung trennt, eine gerade Linie ist. Dieselbe Theorie läßt sich auch anwenden, wenn die Grenze eine gekrümmte Linie darstellt, und sie trifft auch zu, einerlei ob die Grenze die Felder von Verbindungen trennt, die bei ihrem Schmelzpunkt stabil oder instabil sind. 
Für die Untersuchung gekrümmter Grenzlinien dieser Art betrachten wir die Kurve 9-I (Fig. 17). Die Grenzkurve 9-I trennt die Felder A und AS; beide Verbindungen sind bei ihrem Schmelzpunkt stabil. Wenn demnach eine Flüssigkeit der Zusammensetzung $U$ auf der Grenzkurve 9-I abgekühlt wird, so scheiden sich $A$ und AS ab, und die Kristallisationskurve folgt der Grenze I-9. Die Zusammensetzung der Lösung wird gegeben durch Punkte auf $I-9$, die des festen Stoffes durch eine Reihe von Punkten auf der Linie A-AS, deren Lage bestimmt ist durch Verlängerung einer Linie zwischen $U$ und Punkten von $I-9$ bis zum Schnitt der Zusammensetzungslinie A-AS. Weiterhin wird die mittlere Zusammensetzung des festen Stoffes, der sich zwischen zwei beliebigen Kurvenpunkten - etwa $U$ und $V$ - ausgeschieden hat, bestimmt durch Verlängerung einer durch $U$ und $V$ gehenden Linie bis zur Zusammensetzungslinie A-AS. Wenn jetzt die Punkte $U$ und $V$ auf der Kurve 9- $I$ einander näher gebracht werden, so nimmt die durch $U$ und $V$ gezogene Linie die Lage einer Tangente an und wird schließlich wirklich eine Tangente der Grenzkurve; demnach wird die Tangente eines jeden Punktes die Verbindungslinie zweier Phasen schneiden und so die mittlere Zusammensetzung des festen Stoffes angeben, der sich in diesem Punkt ausscheidet. Man erkennt aus der Zeichnung, daß alle Tangenten der Grenze $9-I$ an Punkten zwischẹn $V$ und $U$ die Zusammensetzungslinie A-AS schneiden würden, daB aber alle Tangenten an $9-I$ zwischen 9 und $V$ eine Verlängerung der Zusammensetzungslinie A-AS über A hinaus schneiden. Es folgt demnach, daß auf der Grenze $9-I$ A und AS nebeneinander von $U$ bis $V$ abgeschieden werden, daß aber von $V$ bis $9 \mathrm{~A}$ völlig oder teilweise verschwindet, während AS kristallisiert. Die Grenzkurve 9-I ist von der Art, daß zwei feste Phasen sich nebeneinander auf einem Teil der Kurve ausscheiden und daß auf einem anderen Teil der Kurve eine feste Phase verschwindet, während die andere kristallisiert. Die Änderungen, die auf der Grenze $9-I$ von $V$ bis 9 platzgreifen, sind im wesentlichen dieselben wie auf der Grenze $B^{\prime}-4$ mit Ausnahme der Tatsache, daß im Fall der Grenze 9- $I$ die mittlere Zusammensetzung der sich ausscheidenden festen Phase veränderlich ist, während für die Grenze $B^{\prime}-4$ die mittlere Zusammensetzung der kristallisierenden festen Phase in jedem Punkt konstant ist (Zusammensetzung von $B^{\prime}$ ), weil eine Tangente an jedem Punkt von $B^{\prime}-4$, welches eine gerade Linie ist, dieselbe gerade Linie vorstellt. Es sind noch drei andere Grenzkurven vorhanden, die den 
Grenzen $B^{\prime}-4$ und 9-I ähnlich sind, nämlich $D-17,18-17$ und 18-16. Wenigstens auf einem Teil von jeder dieser drei Kurven verschwindet ein fester Stoff, während der andere kristallisiert. Auf der Grenze D-17 verschwindet $\mathrm{CaO}$ völlig oder teilweise, während $3 \mathrm{CaO} \cdot \mathrm{Al}_{2} \mathrm{O}_{3}$ sich auf dem Wege jeder Kristallisationskurve abscheidet, die dieser Grenze folgt. Auf der Grenze 18-17 verschwindet $\mathrm{CaO}$ von 18 bis $Y$ völlig oder zum Teil, während $3 \mathrm{CaO} . \mathrm{SiO}_{2}$ kristallisiert, und von $Y$ bis 17 scheiden sich $\mathrm{CaO}$ und $3 \mathrm{CaO} . \mathrm{SiO}_{2}$ nebeneinander aus. Auf der Grenze 18-16 kristallisieren von 18 bis $Z 2 \mathrm{CaO} . \mathrm{SiO}_{2}$ und $3 \mathrm{CaO} . \mathrm{SiO}_{2}$ nebeneinander, während von $Z$ bis $162 \mathrm{CaO} . \mathrm{SiO}_{2}$ verschwindet und $3 \mathrm{CaO} . \mathrm{SiO}_{2}$ sich abscheidet.

Es sind demnach fünf Grenzkurven dieser Art vorhanden, nämlich $B^{\prime}-4,9-I, D-16,18-17$ und 18-16, auf denen allen Kristallisationskurven der verschiedenen Arten verlaufen, wie für die Grenze $B^{\prime}-4$ beschrieben ist. Diese verschiedenen Typen der Kristallisationskurven sind folgende: 1. Eine Kristallisationskurve, die bis zu einer Grenzkurve verläuft, auf welcher eine feste Phase teilweise verschwindet, während die andere kristallisiert, endet beim Quintupelpunkt dieser Grenze. 2. Eine Kristallisationskurve, die ebenso wie unter 1 bis zum Quintupelpunkt verläuft, bei der aber in diesem Punkt eine feste Phase völlig verschwindet und die Kristallisationskurve auf einer Grenze bis zu einem anderen Quintupelpunkt fortschreitet. 3. Die Kristallisationskurve, welche bis zu einer Grenze gelangt, auf der eine feste Phase völlig verschwindet, bevor ein Quintupelpunkt erreicht ist, worauf sie die Grenze verläßt und über ein Feld zu einer anderen Grenze gelangt, auf der sie bis zum Quintupelpunkt verläuft.

Welchen dieser drei Typen von Kristallisationskurven eine gegebene Lösung beim Abkühlen folgt, hängt, wie gezeigt worden ist, von der ursprünglichen Zusammensetzung der Lösung ab. Wir werden hier nur die Flächen angeben (Fig. 17), die die Zusammensetzungen der Lösungen für jede dieser drei Arten von Kristalljsationskurven darstellen, sowie die besonderen festen Phasen in der Reihenfolge, wie sie bei einer sich abkühlenden Lösung auftreten oder verschwinden.

1. Es sind vier Flächen vorhanden, die die Zusammensetzungen solcher Lösungen darstellen, welche beim Abkühlen Kristallisationskurven des Typus 1 besitzen. a) Dreieck $\mathrm{C}_{3} \mathrm{~S}_{2}-\mathrm{C}_{2} \mathrm{AS}-\mathrm{C}_{2} \mathrm{~S}$; aus Lösungen, deren Kristallisationskurven die Grenze $B^{\prime}-4$ schneiden, wird sich nacheinander reines $\mathrm{C}_{2} \mathrm{~S}$, sodann gemeinsam $\mathrm{C}_{2} \mathrm{~S}$ und $\mathrm{C}_{3} \mathrm{~S}_{2}$ 
und schließlich $\mathrm{C}_{2} \mathrm{~S}, \mathrm{C}_{3} \mathrm{~S}_{2}$ und $\mathrm{C}_{2} \mathrm{AS}$ abscheiden. b) Dreieck $\mathrm{CAS}_{2}$ AS-A; aus Lösungen, deren Kristallisationskurven die Grenze 9-I schneiden, wird sich nacheinander abscheiden: reines A, A und AS, schließlich . A, AS und $\mathrm{CAS}_{2}$. c) Dreieck $\mathrm{C}_{2} \mathrm{~S}-Z-18$, in dem nacheinander auftreten: $\mathrm{C}_{2} \mathrm{~S}, \mathrm{C}_{2} \mathrm{~S}$ und $\mathrm{C}_{3} \mathrm{~S}$, schließlich $\mathrm{C}_{2} \mathrm{~S}, \mathrm{C}_{3} \mathrm{~S}$ und $\mathrm{C}_{3} \mathrm{~A}$. d) Dreieck $\mathrm{CaO}-\mathrm{X}-\mathrm{C}_{3} \mathrm{~A}$; aus diesen Lösungen, deren Kurve die Grenze 18-17 schneidet, scheiden sich nacheinander ab: $\mathrm{CaO}, \mathrm{CaO}$ und $\mathrm{C}_{3} \mathrm{~S}$, schließlich $\mathrm{CaO}, \mathrm{C}_{3} \mathrm{~S}$ und $\mathrm{C}_{3} \mathrm{~A}$; aus denjenigen Lösungen, deren Kurven die Grenze 17-D treffen, entsteht $\mathrm{CaO}, \mathrm{CaO}$ und $\mathrm{C}_{3} \mathrm{~A}$ und schließlich $\mathrm{CaO}, \mathrm{C}_{3} \mathrm{~A}$ und $\mathrm{C}_{3} \mathrm{~S}$.

2. Acht Flächen stellen die Zusammensetzung solcher Flüssigkeiten dar, die Kristallisationskurven vom Typus 2 liefern. a) Dreieck $\mathrm{C}_{3} \mathrm{~S}_{2}-\mathrm{C}_{2} \mathrm{AS}-4$; aus diesen Flüssigkeiten, deren Kristallisationskurven die Grenzen $B^{\prime}-4$ treffen, scheidet sich nacheinander $a b$ : reines $\mathrm{C}_{2} \mathrm{~S}, \mathrm{C}_{2} \mathrm{~S}$ und $\mathrm{C}_{3} \mathrm{~S}_{2} ; \mathrm{C}_{2} \mathrm{~S}, \mathrm{C}_{3} \mathrm{~S}_{2}$ und $\mathrm{C}_{2} \mathrm{AS} ; \mathrm{C}_{3} \mathrm{~S}_{2}$ und $\mathrm{C}_{2} \mathrm{AS}$ und schlieBlich $\mathrm{C}_{3} \mathrm{~S}_{2}, \mathrm{C}_{2} \mathrm{AS}$ und CS. b) Dreieck $\mathrm{CAS}_{2}-9-\mathrm{AS}$; aus diesen Flüssigkeiten, deren Kristallisationslinien die Grenze 9-I schneiden, tritt nacheinander auf: reines $\mathrm{A}, \mathrm{A}$ und $\mathrm{AS} ; \mathrm{A}, \mathrm{AS}$ und $\mathrm{CAS}_{2} ; \mathrm{CAS}_{2}$ und AS; schließlich $\mathrm{CAS}_{2}$, AS und $\mathrm{S}$. c) Dreieck $\mathrm{C}_{-} \mathrm{C}_{2} \mathrm{~S}-18$; es erscheinen nacheinander: reines $\mathrm{C}_{2} \mathrm{~S}, \mathrm{C}_{2} \mathrm{~S}$ und $\mathrm{CaO} ; \mathrm{C}_{2} \mathrm{~S}$, CaO und $\mathrm{C}_{3} \mathrm{~S}$; $\mathrm{C}_{2} \mathrm{~S}$ und $\mathrm{C}_{3} \mathrm{~S}$ und schließlich $\mathrm{C}_{2} \mathrm{~S}, \mathrm{C}_{3} \mathrm{~S}$ und $\mathrm{C}_{3} \mathrm{~A}$. d) Dreieck $\mathrm{C}_{3} \mathrm{~S}-18-\mathrm{C}$; es scheiden sich nacheinander ab: reines $\mathrm{CaO} ; \mathrm{CaO}$ und $\mathrm{C}_{2} \mathrm{~S} ; \mathrm{C}_{2} \mathrm{~S}$, $\mathrm{CaO}$ und $\mathrm{C}_{3} \mathrm{~S} ; \mathrm{C}_{2} \mathrm{~S}$ und $\mathrm{C}_{3} \mathrm{~S}$, sowie schlieBlich $\mathrm{C}_{2} \mathrm{~S}, \mathrm{C}_{3} \mathrm{~S}$ and $\mathrm{C}_{3} \mathrm{~A}$. e) Dreieck $\mathrm{CaO}-\mathrm{X}-\mathrm{C}_{3} \mathrm{~S}$; es treten nacheinander auf: reines $\mathrm{CaO}$, $\mathrm{CaO}$ und $\mathrm{C}_{2} \mathrm{~S}$; $\mathrm{CaO}, \mathrm{C}_{2} \mathrm{~S}$ und $\mathrm{C}_{3} \mathrm{~S} ; \mathrm{CaO}$ und $\mathrm{C}_{3} \mathrm{~S}$, sowie schließlich $\mathrm{CaO}, \mathrm{C}_{3} \mathrm{~S}$ und $\mathrm{C}_{3} \mathrm{~A}$. f) Dreieck $\mathrm{C}_{2} \mathrm{~S}-\mathrm{Z}-16$; nacheinander kristallisieren: $\mathrm{C}_{2} \mathrm{~S} ; \mathrm{C}_{2} \mathrm{~S}$ and $\mathrm{C}_{3} \mathrm{~S} ; \mathrm{C}_{2} \mathrm{~S}, \mathrm{C}_{3} \mathrm{~S}$ and $\mathrm{C}_{3} \mathrm{~A} ; \mathrm{C}_{2} \mathrm{~S}$ und $\mathrm{C}_{3} \mathrm{~A}$; schließlich $\mathrm{C}_{2} \mathrm{~S}$, $\mathrm{C}_{3} \mathrm{~A}$ und $\mathrm{C}_{5} \mathrm{~A}_{3}$. g) Fläche $\mathrm{C}_{3} \mathrm{~S}-\mathrm{C}_{3} \mathrm{~A}-\mathrm{Y}$; nacheinander treten auf: reines $\mathrm{CaO}$; $\mathrm{CaO}$ und $\mathrm{C}_{3} \mathrm{~S}$; $\mathrm{CaO}, \mathrm{C}_{3} \mathrm{~S}$ and $\mathrm{C}_{3} \mathrm{~A} ; \mathrm{C}_{3} \mathrm{~S}$ und $\mathrm{C}_{3} \mathrm{~A}$ and schließlich $\mathrm{C}_{3} \mathrm{~S}, \mathrm{C}_{3} \mathrm{~A}$ and $\mathrm{C}_{2} \mathrm{~S}$. h) Fläche $\mathrm{C}_{3} \mathrm{~A}-17-Z^{\prime}$; es bilden sich nacheinander: reines $\mathrm{CaO}$; $\mathrm{CaO}$ und $\mathrm{C}_{3} \mathrm{~S}$ oder $\mathrm{CaO}$ und $\mathrm{C}_{3} \mathrm{~A} ; \mathrm{CaO}$, $\mathrm{C}_{3} \mathrm{~S}$ und $\mathrm{C}_{3} \mathrm{~A} ; \mathrm{C}_{3} \mathrm{~S}$ and $\mathrm{C}_{3} \mathrm{~A} ; \mathrm{C}_{3} \mathrm{~S}, \mathrm{C}_{3} \mathrm{~A}$ and $\mathrm{C}_{2} \mathrm{~S} ; \mathrm{C}_{2} \mathrm{~S}$ und $\mathrm{C}_{3} \mathrm{~A}$, sowie schließlich $\mathrm{C}_{3} \mathrm{~A}, \mathrm{C}_{2} \mathrm{~S}$ und $\mathrm{C}_{5} \mathrm{~A}_{3}$.

3. Vier Flächen stellen die Zusammensetzungen solcher Flüssigkeiten dar, die Kristallisationskurven des dritten Typus besitzen. a) Dreieck $\mathrm{C}_{3} \mathrm{~S}_{2}-4-B^{\prime}$; es scheiden sich nacheinander ab: reines $\mathrm{C}_{2} \mathrm{~S}$; $\mathrm{C}_{2} \mathrm{~S}$ und $\mathrm{C}_{3} \mathrm{~S}_{2}$; reines $\mathrm{C}_{3} \mathrm{~S}_{2} ; \mathrm{C}_{3} \mathrm{~S}_{2}$ und $\mathrm{CS}$; schlieBlich $\mathrm{C}_{3} \mathrm{~S}_{2}$, CS und $\mathrm{C}_{2} \mathrm{AS}$. b) Fläche $9-U-V$; es kristallisieren nacheinander: reines $\mathrm{A}$; $A$ und $A S$; reines AS; AS und $\mathrm{CAS}_{2}$, sowie schließlich AS, $\mathrm{CAS}_{2}$ und $\mathrm{S}$. c) Dreieck $\mathrm{C}_{3} \mathrm{~S}-18-Y$; wenn die Kristallisationskurve die 
Grenze zwischen 18 und $\mathrm{C}$ trifft, so scheiden sich nacheinander ab: $\mathrm{CaO} ; \mathrm{CaO}$ und $\mathrm{C}_{2} \mathrm{~S} ; \mathrm{CaO}, \mathrm{C}_{2} \mathrm{~S}$ und $\mathrm{C}_{3} \mathrm{~S} ; \mathrm{CaO}-\mathrm{C}_{3} \mathrm{~S}$; reines $\mathrm{C}_{3} \mathrm{~S} ; \mathrm{C}_{3} \mathrm{~S}$ und $\mathrm{C}_{2} \mathrm{~S}$; sowie schließlich $\mathrm{C}_{3} \mathrm{~S}, \mathrm{C}_{2} \mathrm{~S}$ und $\mathrm{C}_{3} \mathrm{~A}$; oder wenn die Lösungen auf die Grenze zwischen 18 und $Y$ treffen, so tritt auf: reines $\mathrm{CaO}$; $\mathrm{CaO}$ und $\mathrm{C}_{3} \mathrm{~S}$; reines $\mathrm{C}_{3} \mathrm{~S} ; \mathrm{C}_{3} \mathrm{~S}$ und $\mathrm{C}_{2} \mathrm{~S}$, sowie schließlich $\mathrm{C}_{3} \mathrm{~S}, \mathrm{C}_{2} \mathrm{~S}$ und $\mathrm{C}_{3} \mathrm{~A}$. d) Fläche $\mathrm{C}_{3} \mathrm{~A}-17-D$; nacheinander kristallisieren: reines $\mathrm{CaO}$; $\mathrm{CaO}$ und $\mathrm{C}_{3} \mathrm{~A}$; reines $\mathrm{C}_{3} \mathrm{~A} ; \mathrm{C}_{3} \mathrm{~A}$ und $\mathrm{C}_{2} \mathrm{~S}$ oder $\mathrm{C}_{3} \mathrm{~A}$ and $\mathrm{C}_{5} \mathrm{~A}_{3}$, sowie schließlich $\mathrm{C}_{3} \mathrm{~A}, \mathrm{C}_{2} \mathrm{~S}$ und $\mathrm{C}_{5} \mathrm{~A}_{3}$.

Allgemeine Folgerungen über die Art der Kristallisation in ternären Lösungen von $\mathrm{CaO}-\mathrm{Al}_{2} \mathrm{O}_{3}-\mathrm{SiO}_{2}$. Die Art der Kristallisation einer ternären Flüssigkeit hängt $a b$ von der ursprünglichen Zusammensetzung der Lösung, sowie von der Natur der Grenzkurve, welche die Kristallisationskurve schneidet. Zwei Klassen von Grenzkurven sind vorhanden:

1. Grenzkurven, auf denen zwei Phasen nebeneinander kristallisieren. Eine Tangente an irgendeinen Punkt einer solchen Grenzkurve wird die Verbindungslinie der Zusammensetzungen dieser beiden Phasen schneiden. Zu dieser Klasse gehören alle Grenzkurven, deren eines Ende ein eutektischer Punkt ist, z. B. in Fig. 17 die Grenzen $A-2, I-1, M-5,11-7$, $16-15,13-14, F-14$ usw.

2. Grenzkurven, auf denen eine Phase kristallisiert, während die andere zum Teil oder vollständig verschwindet. Eine an irgendeinen Punkt einer derartigen Grenzkurve gezogene Tangente wird die Verlängerung der Verbindungslinie der Zusammensetzungen von beiden Phasen schneiden. Zu dieser Klasse gehören die Grenzen $9-V, B^{\prime}-4, Z-16,18-Z$ und $D-17$.

Wenn man demnach die ursprüngliche Zusammensetzung einer ternären Flüssigkeit und die Art der Grenzkurve kennt, auf welche die Kristallisation beim Abkühlen der Lösung zuschreitet, so kann man aus einer Untersuchung des Gleichgewichtsdiagrammes genau feststellen, welche Phasen sich abscheiden, in welcher Reihenfolge die Kristallisation stattfindet, und welches das Enảprodukt der Kristallisation ist.

Das letzte Produkt der Kristallisation einer ternären Lösung von $\mathrm{CaO}, \mathrm{Al}_{2} \mathrm{O}_{3}$ und $\mathrm{SiO}_{2}$ wird immer aus drei festen Phasen bestehen, deren Stabilitätsgebiete nebeneinander liegen. Es werden dieselben drei festen Phasen das Endprodukt der Kristallisation einer jeden Lösung sein, deren Zusammensetzung in dem Dreieck 
liegt, das gebildet wird aus den Linien, die die Zusammensetzungen dieser drei Phasen miteinander verbinden.

Die Kenntnis der angeführten Tatsachen ist für das Studium eines ternären Systemes von beträchtlicher Bedeutung. Wenn man die Temperaturen der Grenzkurve und des Quintupelpunktes nach den Knicken etwa einer Abkühlungslinie feststellt, so ist es durchaus erforderlich, die Ursache eines derartigen Knickes (Energieänderung) zu kennen. Gewöhnlich geht man von einer ungesättigten Lösung aus, so daß der erste Knick der Erstarrungspunkt der ersten Phase ist; der zweite Knick tritt beim Schnitt der Grenzkurve auf und der dritte Knick stellt in einem solchen Fall den Quintupelpunkt dar. Lösungen von Silikatgemischen zeigen jedoch derartige Unterkühlungen, daß eine Zeit-Abkühlungskurve wertlos ist; deswegen wird bei Experimentaluntersuchungen die Zeit-Erhitzungskurve benutzt, auf welcher die festen Phasen in umgekehrter Reihenfolge wie bei der Kristallisation schmelzen. Selbst bei Erhitzungskurven sind jedoch die Knicke, die man für das Schmelzen gewisser Silikate erhält, nicht scharf; in solchen Fällen ist das Abschreckverfahren, nach dem man die optischen Eigenschaften einer Phase sowie ihre Schmelztemperatur bestimmen kann, besser geeignet.

Mit dem Abschreckungsverfahren haben wir die Beobachtungen gemacht, aus denen abzuleiten ist, was unter idealen Bedingungen der Kristallisation beim Abkühlen der Lösungen von $\mathrm{CaO}, \mathrm{Al}_{2} \mathrm{O}_{3}$ und $\mathrm{SiO}_{2}$ stattfinden wird. Die so erhaltenen Ergebnisse, die die vorhandenen Phasen und ihre Temperatur-Stabilitätsgebiete für gewisse Flüssigkeiten liefern, geben Beispiele von allen Arten der beschriebenen Kristallisationskurven; sie sind in den Tabellen 21-24 mitgeteilt.

Die Daten von Tabelle 21 entsprechen dem Typus der Kristallisationskurve, die einer Grenzkurve direkt bis zum eutektischen Punkte folgt. So beginnt bei der Lösung der Zusammensetzung $38 \% \mathrm{CaO}, 51.5 \% \mathrm{Al}_{2} \mathrm{O}_{3}, 10.5 \% \quad \mathrm{SiO}_{2}$ die Kristallisation von $2 \mathrm{CaO}$ $\mathrm{Al}_{2} \mathrm{O}_{3} \cdot \mathrm{SiO}_{2}$ bei einer Temperatur zwischen $1540^{\circ}$ und $1520^{\circ}$; bei $1515^{0}$ hat die Kristallisationskurve die Grenze 13-12 (Fig. 17) erreicht, auf der $2 \mathrm{CaO} \cdot \mathrm{Al}_{2} \mathrm{O}_{3} \cdot \mathrm{SiO}_{2}$ und $\mathrm{CaO} \cdot \mathrm{Al}_{2} \mathrm{O}_{3}$ nebeneinander kristallisieren, bis bei $1505^{\circ}$ der eutektische Punkt 12 erreicht ist, worauf dann $2 \mathrm{CaO} \cdot \mathrm{Al}_{2} \mathrm{O}_{3} . \mathrm{SiO}_{2}, \mathrm{CaO} . \mathrm{Al}_{2} \mathrm{O}_{3}$ und $3 \mathrm{CaO} .5 \mathrm{Al}_{2} \mathrm{O}_{3}$ nebeneinander sich abscheiden. 
Tabelle 21.

Beispiele der kristallisierten Phasen, die auf der Kristallisationskurve vom einfachsten Typus in einem ternären System auftreten.

\begin{tabular}{|c|c|c|c|c|}
\hline $\begin{array}{r}\text { Zusal } \\
\text { ir } \\
\mathrm{CaO}\end{array}$ & $\begin{array}{l}\text { amense } \\
\text { Gew.- } \\
\mathrm{Al}_{2} \mathrm{O}_{3}\end{array}$ & $\begin{array}{l}\text { zung } \\
1 / 0 \\
\mathrm{SiO}_{3}\end{array}$ & $\begin{array}{l}\text { Tempe- } \\
\text { ratur }\end{array}$ & Vorhandene Phasen \\
\hline 38 & 51.5 & 10.5 & $\begin{array}{l}1540 \\
1520 \\
1515 \\
1505\end{array}$ & $\begin{array}{l}\text { Glas } \\
\text { Glas + } 2 \mathrm{CaO} \cdot \mathrm{Al}_{2} \mathrm{O}_{3} \cdot \mathrm{SiO}_{2} \\
\text { Glas + } 2 \mathrm{CaO} \cdot \mathrm{Al}_{2} \mathrm{O}_{3} \cdot \mathrm{SiO}_{2}+\mathrm{CaO} \cdot \mathrm{Al}_{2} \mathrm{O}_{3} \\
\text { Glas + } \\
\quad 2 \mathrm{CaO} \cdot \mathrm{Al}_{2} \mathrm{O}_{3} \cdot \mathrm{SiO}_{2}+\mathrm{CaO} \cdot \mathrm{Al}_{2} \mathrm{O}_{3}+3 \mathrm{CaO} . \\
\quad 5 \mathrm{Al}_{2} \mathrm{O}_{3}\end{array}$ \\
\hline 52 & 38 & 10 & $\begin{array}{l}1470 \\
1450 \\
1410 \\
1340 \\
1330\end{array}$ & $\begin{array}{l}\text { Glas } \\
\text { Glas }+\mathrm{Ca}_{2} \mathrm{SiO}_{4} \\
\text { Glas }+\mathrm{Ca}_{2} \mathrm{SiO}_{4} \\
\text { Glas }+\mathrm{Ca}_{2} \mathrm{SiO}_{4}+5 \mathrm{CaO}_{3} \mathrm{Al}_{2} \mathrm{O}_{3} \\
\mathrm{Ca}_{2} \mathrm{SiO}_{4}+5 \mathrm{CaO} .3 \mathrm{Al}_{2} \mathrm{O}_{3}+\mathrm{CaO} \cdot \mathrm{Al}_{2} \mathrm{O}_{3}\end{array}$ \\
\hline 52.5 & 42.5 & 5 & $\begin{array}{l}1405 \\
1380 \\
1350 \\
1325\end{array}$ & $\begin{array}{l}\text { Glas } \\
\text { Glas }+3 \mathrm{CaO} \cdot \mathrm{Al}_{2} \mathrm{O}_{3} \\
\text { Glas }+3 \mathrm{CaO} \cdot \mathrm{Al}_{2} \mathrm{O}_{3}+5 \mathrm{CaO} \cdot 3 \mathrm{Al}_{2} \mathrm{O}_{3} \\
3 \mathrm{CaO} \cdot \mathrm{Al}_{2} \mathrm{O}_{3}+5 \mathrm{CaO} \cdot 3 \mathrm{Al}_{2} \mathrm{O}_{3}+\mathrm{Ca}_{2} \mathrm{SiO}_{4}\end{array}$ \\
\hline
\end{tabular}

Die Daten von Tabelle 22 entsprechen dem Typus der Kristallisation, bei dem eine Grenzkurve bis zu einem Quintupelpunkt nicht eutektischer Natur verfolgt wird. Soweit die Zahlen erkennen lassen, ist diese Art der Kurve dieselbe, wie bei Tabelle 21; nur besteht der Unterschied, daß nach Erreichung der Temperatur $1880^{\circ} \pm 5^{0}$ - des Quintupelpunktes (13) - die Verbindung $2 \mathrm{CaO} \cdot \mathrm{Al}_{2} \mathrm{O}_{3} \cdot \mathrm{SiO}_{2}$ zum Teil verschwindet, während $2 \mathrm{CaO} . \mathrm{SiO}_{2}$ kristallisiert.

\section{Tabelle 22.}

Beispiele von kristallisierten Phasen, die auf einer Kristallisationskurve auftreten, welche in einem nicht eutektischen Quintupelpunkt endet.

\begin{tabular}{|c|c|c|c|c|}
\hline \multicolumn{3}{|c|}{$\begin{array}{l}\text { Zusammensetzung } \\
\text { in Gew.- } \%\end{array}$} & $\begin{array}{l}\text { Tempe- } \\
\text { ratur }\end{array}$ & Vorhandene Phasen \\
\hline 45 & 43.5 & 11.5 & $\begin{array}{l}1490 \\
1450 \\
1385 \\
1375\end{array}$ & $\begin{array}{l}\text { Glas }+2 \mathrm{CaO} \cdot \mathrm{Al}_{2} \mathrm{O}_{3} \cdot \mathrm{SiO}_{2} \\
\text { Glas }+2 \mathrm{CaO} \cdot \mathrm{Al}_{2} \mathrm{O}_{3} \cdot \mathrm{SiO}_{2}+\mathrm{CaO} \cdot \mathrm{Al}_{2} \mathrm{O}_{3} \\
\text { Glas }+2 \mathrm{CaO} \cdot \mathrm{Al}_{2} \mathrm{O}_{3} \cdot \mathrm{SiO}_{2}+\mathrm{CaO} \cdot \mathrm{Al}_{2} \mathrm{O}_{3}+\mathrm{Ca}_{2} \mathrm{SiO}_{4} \\
2 \mathrm{CaO} \cdot \mathrm{Al}_{2} \mathrm{O}_{3} \cdot \mathrm{SiO}_{2}+\mathrm{CaO} \cdot \mathrm{Al}_{2} \mathrm{O}_{3}+\mathrm{Ca}_{2} \mathrm{SiO}_{4}\end{array}$ \\
\hline 47.5 & 37.5 & 15 & $\begin{array}{l}1500 \\
1475 \\
1400 \\
1350\end{array}$ & $\begin{array}{l}\text { Glas }+2 \mathrm{CaO} \cdot \mathrm{Al}_{2} \mathrm{O}_{3} \cdot \mathrm{SiO}_{2} \\
\text { Glas }+2 \mathrm{CaO} \cdot \mathrm{Al}_{2} \mathrm{O}_{3} \cdot \mathrm{SiO}_{2}+\mathrm{Ca}_{2} \mathrm{SiO}_{4} \\
\text { Glas }+2 \mathrm{CaO} \cdot \mathrm{Al}_{2} \mathrm{O}_{3} \cdot \mathrm{SiO}_{2}+\mathrm{Ca}_{2} \mathrm{SiO}_{4} \\
2 \mathrm{CaO} \cdot \mathrm{Al}_{2} \mathrm{O}_{3} \cdot \mathrm{SiO}_{2}+\mathrm{Ca}_{2} \mathrm{SiO}_{4}+\mathrm{CaO} \cdot \mathrm{Al}_{2} \mathrm{O}_{3}\end{array}$ \\
\hline
\end{tabular}


Die Zahlen von Tabelle 23 entsprechen dem Typus der Kristallisationskurve, bei welchem eine feste Phase bei einem Quintupelpunkt vollständig verschwindet. So hat für eine Lösung der Zusammensetzung $48.5 \% \mathrm{CaO}, 12 \% \quad \mathrm{Al}_{2} \mathrm{O}_{3}$ und $39.5 \% \quad \mathrm{SiO}_{2}$ bei der Temperatur $1340^{\circ}$ die Kristallisationskurve die Grenzkurve $S-4$ erreicht, worauf dann $2 \mathrm{CaO} \cdot \mathrm{SiO}_{2}$ und $2 \mathrm{CaO} \cdot \mathrm{Al}_{2} \mathrm{O}_{3} \cdot \mathrm{SiO}_{2}$ nebeneinander kristallisieren; bei $1330^{\circ}$ ist sie durch den Quintupelpunkt 4 hindurchgegangen und schreitet auf der Grenze 4-6 fort, wo sich dann $2 \mathrm{CaO} \cdot \mathrm{Al}_{2} \mathrm{O}_{3} \cdot \mathrm{SiO}_{2}$ und $3 \mathrm{CaO} .2 \mathrm{SiO}_{2}$ nebeneinander abscheiden; bei $1310^{\circ}$ hat sie den eutektischen Punkt 6 erreicht und die Lösung verfestigt sich $\mathrm{zu} \mathrm{CaO} . \mathrm{SiO}_{2}, 3 \mathrm{CaO} .2 \mathrm{SiO}_{2}$ und $2 \mathrm{CaO} \cdot \mathrm{Al}_{2} \mathrm{O}_{3} . \mathrm{SiO}_{2}$.

Tabelle 23.

Beispiele von kristallisierten Phasen auf einer Kristallisationskurve, die durch einen Quintupelpunkt hindurchgeht und in einem eutektischen Punkt endet.

\begin{tabular}{|c|c|c|c|c|}
\hline \multicolumn{3}{|c|}{$\begin{array}{l}\text { Zusammensetzung } \\
\text {, in Gew.- } \%\end{array}$} & \multirow[t]{2}{*}{$\begin{array}{c}\text { Tempe- } \\
\text { ratur }\end{array}$} & \multirow[t]{2}{*}{ Vorhandene Phasen } \\
\hline $\mathrm{CaO}$ & $\mathrm{Al}_{2} \mathrm{O}_{3}$ & $\mathrm{SiO}_{2}$ & & \\
\hline 48.5 & 12 & 39.5 & $\begin{array}{l}1340 \\
1330 \\
1310\end{array}$ & $\begin{array}{l}\text { Glas }+2 \mathrm{CaO} \cdot \mathrm{Al}_{2} \mathrm{O}_{3} \cdot \mathrm{SiO}_{2}+2 \mathrm{CaO} \cdot \mathrm{SiO}_{2} \\
\text { Glas }+2 \mathrm{CaO} \cdot \mathrm{Al}_{2} \mathrm{O}_{3} \cdot \mathrm{SiO}_{2}+3 \mathrm{CaO} \cdot 2 \mathrm{SiO}_{2} \\
\mathrm{CaOSiO}+2 \mathrm{CaO} \cdot \mathrm{Al}_{2} \mathrm{O}_{3} \cdot \mathrm{SiO}_{2}+3 \mathrm{CaO} \cdot 2 \mathrm{SiO}_{2}\end{array}$ \\
\hline 60 & 32.5 & 7.5 & $\begin{array}{l}1480 \\
1460 \\
1440\end{array}$ & $\begin{array}{l}\mathrm{Glas}+\mathrm{CaO} \\
\mathrm{Glas}+3 \mathrm{CaO} \cdot \mathrm{Al}_{2} \mathrm{O}_{3}+3 \mathrm{CaO} \cdot \mathrm{SiO}_{2} \\
\mathrm{Glas}+3 \mathrm{CaO} \cdot \mathrm{Al}_{2} \mathrm{O}_{3}+\mathrm{Ca}_{2} \mathrm{SiO}_{4}\end{array}$ \\
\hline 59 & 32.5 & 8.5 & $\begin{array}{l}1470 \\
1455 \\
1440\end{array}$ & $\begin{array}{l}\mathrm{Glas}+3 \mathrm{CaO} \cdot \mathrm{SiO}_{2} \\
\mathrm{Glas}+3 \mathrm{CaO} \cdot \mathrm{SiO}_{2}+3 \mathrm{CaO} \cdot \mathrm{Al}_{2} \mathrm{O}_{3}+\mathrm{Ca}_{2} \mathrm{SiO}_{4} \\
\text { Glas }+3 \mathrm{CaO} \cdot \mathrm{Al}_{2} \mathrm{O}_{3}+\mathrm{Ca}_{2} \mathrm{SiO}_{4}\end{array}$ \\
\hline
\end{tabular}

Die Zahlen von Tabelle 24 entsprechen dem Typus der Kristallisationskurve, bei der ein fester Stoff auf einer Grenzkurve völlig verschwindet und die Kurve durch ein Feld nach einer anderen Grenzkurve verläuft. In einer Lösung der Zusammensetzung 53\% $\mathrm{CaO}, 4 \% \mathrm{Al}_{2} \mathrm{O}_{3}, 43 \% \mathrm{SiO}_{2}$ kristallisiert bei $1420^{\circ} 2 \mathrm{CaO} . \mathrm{SiO}_{2}$; bei $1415^{\circ}$ hat die Kristallisationskurve die Grenze $B^{\prime}-4$ erreicht, wo $2 \mathrm{CaO} . \mathrm{SiO}_{2}$ verschwindet, und die Abscheidung von $3 \mathrm{CaO}_{2} \mathrm{SiO}_{2}$ beginnt; bei $1410^{\circ}$ ist $2 \mathrm{CaO} . \mathrm{SiO}_{2}$ völlig verschwunden, die Kristallisationslinie hat die Grenze $B^{\prime}-4$ verlassen und ist in das Feld $B^{\prime}-4-6-B$ eingetreten, auf dem reines $3 \mathrm{CaO}_{2} 2 \mathrm{SiO}_{2}$ sich abscheidet. 


\section{Tabelle 24.}

Beispiele von kristallisierten Phasen auf einer Kristallisationskurve, die durch ein Feld verläuft.

\begin{tabular}{|c|c|c|c|c|}
\hline $\mathrm{CaO}$ & $\begin{array}{l}\text { Gewense } \\
\mathrm{Al}_{2} \mathrm{O}_{3} \\
\end{array}$ & $\mathrm{SiO}_{2}$ & $\begin{array}{l}\text { Tempe- } \\
\text { ratur }\end{array}$ & Vorhandene Phasen \\
\hline 53 & 4 & 43 & $\begin{array}{l}1420 \\
1415 \\
1410\end{array}$ & $\begin{array}{l}\text { Glas }+2 \mathrm{CaO}_{\mathrm{SiO}} \\
\text { Glas }+2 \mathrm{CaO} . \mathrm{SiO}_{2}+3 \mathrm{CaO} .2 \mathrm{SiO}_{2} \\
\text { Glas }+3 \mathrm{CaO} .2 \mathrm{SiO}_{2}\end{array}$ \\
\hline 14 & 38.5 & 47.5 & $\begin{array}{l}1574 \\
1570 \\
1550 \\
1545\end{array}$ & $\begin{array}{l}\text { Glas }+\mathrm{Al}_{2} \mathrm{O}_{3} \\
\text { Glas }+\mathrm{Al}_{2} \mathrm{O}_{3}+\mathrm{Al}_{2} \mathrm{SiO}_{5} \\
\text { Glas }+\mathrm{Al}_{2} \mathrm{O}_{3}+\mathrm{Al}_{2} \mathrm{SiO}_{5} \\
\text { Glas }+\mathrm{Al}_{2} \mathrm{SiO}_{5}\end{array}$ \\
\hline 63 & 25 & 12 & $\begin{array}{l}1610 \\
1500 \\
1475 \\
1425\end{array}$ & $\begin{array}{l}\text { Glas }+\mathrm{CaO}+3 \mathrm{CaO} \cdot \mathrm{SiO}_{2} \\
\text { Glas }+3 \mathrm{CaO} \cdot \mathrm{SiO}_{2} \\
\text { Glas }+3 \mathrm{CaO} \cdot \mathrm{SiO}_{2}+\mathrm{Ca}_{2} \mathrm{SiO}_{4} \\
\text { Glas }+\mathrm{Ca}_{2} \mathrm{SiO}_{4}+3 \mathrm{CaO} \cdot \mathrm{Al}_{2} \mathrm{O}_{3}\end{array}$ \\
\hline
\end{tabular}

\section{Die Anwendung der Gleichgewichte im System $\mathrm{CaO}-\mathrm{Al}_{2} \mathrm{O}_{3}-\mathrm{SiO}_{2}$ auf Probleme, bei denen diese Oxyde in Frage kommen.}

Es ist nicht unsere Absicht, hier alle möglichen Anwendungen des Gleichgewichtsdiagrammes von $\mathrm{CaO}, \mathrm{Al}_{2} \mathrm{O}_{3}$ und $\mathrm{SiO}_{2}$ auf Probleme zu besprechen, an denen diese Oxyde teilnehmen. Wir wollen lediglich zeigen, wie dies Diagramm angewendet werden kann, und wir möchten kurz seine Anwendung auf die Untersuchung von Portlandzementklinkern und gewisse geologische Fragen betrachten. ${ }^{1}$

Um dies Diagramm zu erhalten, war es notwendig, die Gleichgewichtsbedingungen für die Existenz der Komponenten sowie auch von ihren verschiedenen Verbindungen, sei es in reinem Zustande oder in Mischungen, festzustellen. Diese Gleichgewichtsbedingungen sind dargestellt worden in Diagrammen, die hauptsächlich die Verhältnisse der Flüssigkeiten wiedergeben; mit anderen Worten: wir haben die Bedingungen dargelegt, unter denen die festen Komponenten und Verbindungen im Gleichgewicht mit Lösungen vorhanden sind. Die Zeichnung Fig. 17 stellt dar, wie diese Komponenten und Verbindungen aus Lösungen kristallisieren; sie ermöglicht uns, die Reihenfolge der Kristallisation sowie das Endprodukt festzustellen, wenn die Lösungen völlig kristallisiert sind.

1 Diese Anwendungen werden ausführlicher in Arbeiten besprochen werden, die demnächst erscheinen sollen. 
Diese letzten Produkte der Kristallisation von Lösungen des Systemes $\mathrm{CaO}, \mathrm{Al}_{2} \mathrm{O}_{3}, \mathrm{SiO}_{2}$, die die einzigen wesentlichen Daten sind, welche man braucht, um die meisten Probleme zu lösen, die bei aiesem System auftreten, werden auf der Zeichnung von Fig. 19

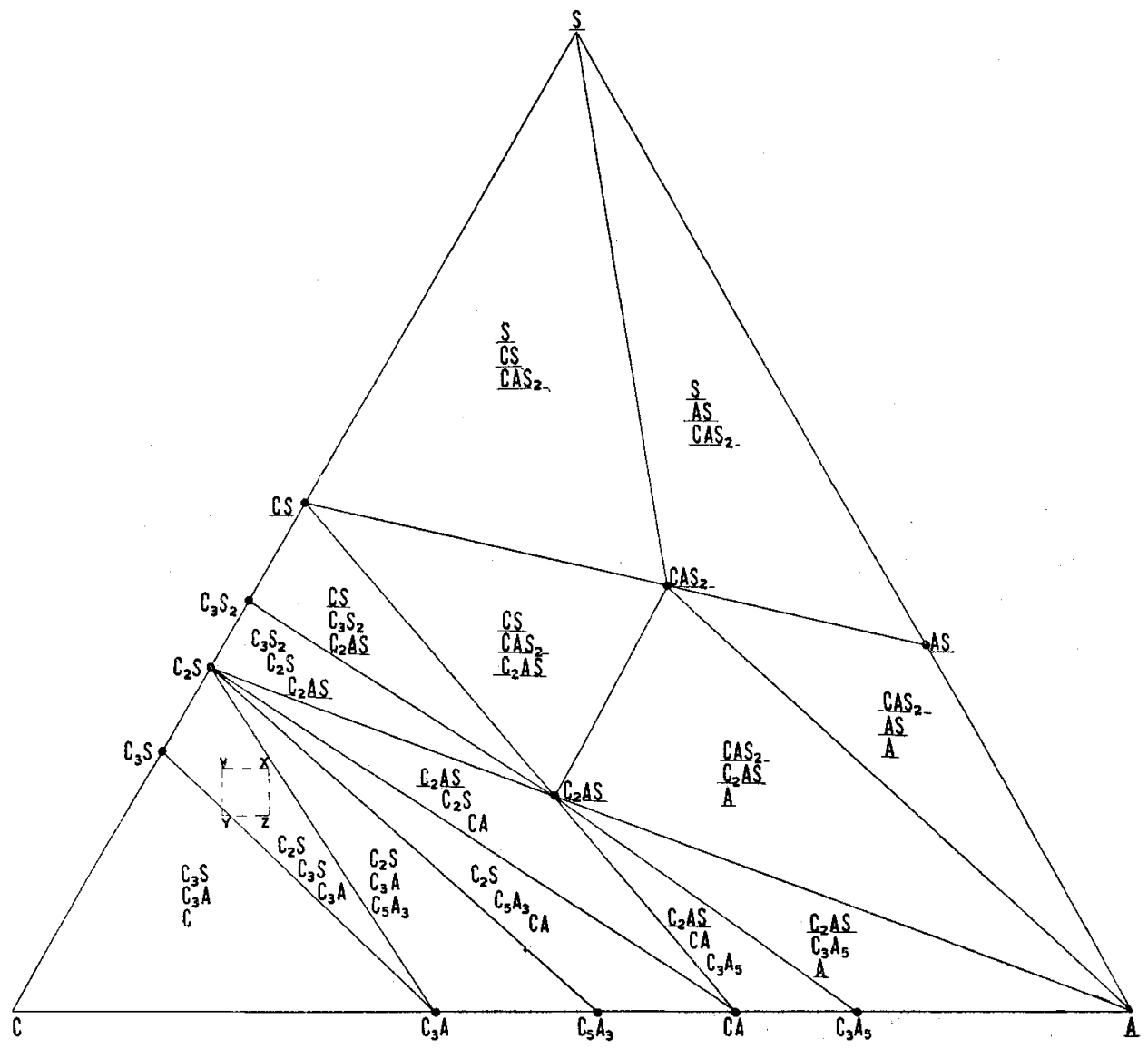

Fig. 19.

Endprodukte der Kristallisation von Lösungen aus $\mathrm{CaO} \cdot \mathrm{Al}_{2} \mathrm{O}_{8}-\mathrm{SiO}_{2}$.

dargestellt, die, wie man sieht, aus einer großen Anzahl dreieckiger Flächen besteht. Jedes dieser Dreiecke gibt alle möglichen Gemische der drei Verbindungen wieder, deren Zusammensetzungen durch die Spitzen der Dreiecke ausgedrückt werden. Wenngleich drei bestimmte Verbindungen gemeinschaftlich nur in einem Dreieck bestehen können, so kann doch, wie man aus der Zeichnung sieht, 
eine jede dieser drei Verbindungen in einer Anzahl verschiedener Dreiecke aufgefunden werden.

Wenn man die Angaben der Fig. 19 auf irgendeine Frage anwendet, bei der ein Teil dieser Zeichnung oder auch die ganze Zeichnung in Betracht kommt, so muß man sich daran erinnern, daß diese Darstellung Gleichgewichtsbedingungen entspricht; dies Diagramm stellt also die Komponenten und die Verbindungen so dar, wie sie nebeneinander in Mischungen vorkommen, wenn hinreichend viel Zeit vorhanden ist, daß alle Reaktionen vollständig verlaufen. Bei den Fragen, auf die wir dies Diagramm anzuwenden wünschen, wissen wir aber, daß die gegebenen Reaktionen nicht immer vollständig verlaufen und $d a ß$ die Gleichgewichtsbedingungen nicht erreicht werden. Daraus folgt jedọch nicht, daß die Anwendung dieses Diagrammes auf solche Fragen ohne Wert ist; vielmehr muß man folgern, daß dies Diagramm untersucht werden muß, um die möglichen Bedingungen festzustellen, die eintreten, wenn das Gleichgewicht nicht erreicht wird. Wenn man.z. B. ein Materialgemisch hat, dessen Gehalt an $\mathrm{CaO}, \mathrm{Al}_{2} \mathrm{O}_{3}$ und $\mathrm{SiO}_{2}$ irgendeinem Punkte der Zeichnung Fig. 19 entspricht, so könnte man erwarten, daß die vorhandenen Verbindungen dieser drei Oxyde in weitem Umfange den Verbindungen jenes kleinen Dreiecks entsprechen, in dem der betreffende Punkt liegt; wenn jedoch andere Verbindungen vorhanden sind, so würde man erwarten, daß diese Verbindungen in den benachbarten Dreiecken liegen.

\section{Anwendung auf die Portlandzement-Klinker.}

Es ist bekannt, daß Portlandzement aus den reinen Oxyden $\mathrm{CaO}, \mathrm{Al}_{2} \mathrm{O}_{3}$ und $\mathrm{SiO}_{2}$ hergestellt werden kann. Nach den Angaben von Richardson kann der Klinker aus Gemischen dieser drei Oxyde hergestellt werden, deren Zusammensetzung durch die Punkte der Fläche $V-X-Z-Y$ (Fig. 19) zur Darstellung kommen; diese Fläche liegt fast völlig im Dreieck $\mathrm{C}_{2} \mathrm{~S}-\mathrm{C}_{3} \mathrm{~S}-\mathrm{C}_{3} \mathrm{~A}$. Demnach würde ein Zementklinker aus reinem $\mathrm{CaO}, \mathrm{Al}_{2} \mathrm{O}_{3}$ und $\mathrm{SiO}_{2}$, der hinreichend lange bei einer genügend hohen Temperatur gebrannt ist, aus den drei Verbindungen $2 \mathrm{CaO} . \mathrm{SiO}_{2}, 3 \mathrm{CaO} . \mathrm{SiO}_{2}$ und $3 \mathrm{CaO} . \mathrm{Al}_{2} \mathrm{O}_{3}$ bestehen. Wenn man sich jedoch dem Gleichgewicht nur genähert hat, ohne es zu erreichen, so müßte man erwarten, außer diesen drei Verbindungen noch freies $\mathrm{CaO}$ oder die Verbindung $5 \mathrm{CaO} .3 \mathrm{Al}_{2} \mathrm{O}_{3}$ oder beide zu finden.

Käuflicher Portlandzement-Klinker hat einen Gehalt an $\mathrm{CaO}$, 
$\mathrm{Al}_{2} \mathrm{O}_{3}$ und $\mathrm{SiO}_{2}$ von über $90 \%$; seine Herstellung geschieht so, daß man eine Annäherung an Gleichgewichtsverhältnisse erwarten kann, so daß es möglich erscheint, aus Fig. $19 \mathrm{zu}$ entnehmen, welche Verbindungen von $\mathrm{CaO}, \mathrm{Al}_{2} \mathrm{O}_{3}$ und $\mathrm{SiO}_{2}$ gebildet werden, wenn man die Rohmaterialien für den Portlandzement brennt. Aus diesen Darstellungen haben wir vorausgesagt, daß Portlandzement-Klinker zum großen Teil aus $3 \mathrm{CaO} . \mathrm{SiO}_{2}, 2 \mathrm{CaO} . \mathrm{SiO}_{2}, 3 \mathrm{CaO} . \mathrm{Al}_{2} \mathrm{O}_{3}, 5 \mathrm{CaO} .3 \mathrm{Al}_{2} \mathrm{O}_{3}$ und vielleicht einer kleinen Menge von freiem Kalk bestehen; die dann folgende Untersuchung im Bureau of Standards in Pittsburg 1 hat die Anwendbarkeit der entwickelten Gesichtspunkte auf die Klinker des Handels gezeigt und diese Voraussage völlig bestätigt. Als wir uns über die wahrscheinliche Konstitution von Portlandzement-Klinkern äußerten, glaubten wir - was sich später auch bestätigte - daß andere Komponenten außer $\mathrm{CaO}, \mathrm{Al}_{2} \mathrm{O}_{3}$ und $\mathrm{SiO}_{2}$ praktisch vernachlässigt werden könnten. Wir meinen allerdings hiermit nicht, daß andere Komponenten wie $\mathrm{MgO}$, FeO usw., keine Bedeutung für Zement oder zementartige Materialien besitzen, sondern lediglich, daß diese Komponenten nur in so kleinen Mengen in dem rohen Zementmaterial vorhanden sind, daß wahrscheinlich die Klinker zum großen Teil aus Verbindungen von reinem Kalk, Tonerde und Kieselsäure bestehen. Es ist jedoch nicht.direkt zulässig, die Daten der Fig. 19 auf die Probleme des Systemes $\mathrm{CaO}, \mathrm{Al}_{2} \mathrm{O}_{3}$, $\mathrm{SiO}_{2}$ anzuwenden, wenn man nicht sicher ist, daß die anderen vorhandenen Komponenten vernachlässigt werden können und daß wenigstens angenähert die Gleichgewichts bedingungen erreicht worden sind.

\section{Anwendung der Ergebnisse auf einige geologische Fragen.}

Bei der Anwendung der bei dieser Untersuchung erhaltenen Ergebnisse auf Fragen, welche die natürlichen Mineralien aus $\mathrm{CaO}$, $\mathrm{Al}_{2} \mathrm{O}_{3}$ und $\mathrm{SiO}_{2}$ betreffen, $\mathrm{muB}$ man vorsichtig vorgehen, weil die Bedingungen der natürlichen Bildung sehr von den Verhältnissen abweichen, unter denen dies System im Laboratorium untersucht worden ist. Es sind zwei Hauptunterschiede vorhanden: 1. unter geologischen Bedingungen finden die Reaktionen unter hohem Druck statt (der überdies wahrscheinlich weder konstant noch gleichförmig ist); 2. die verwickelte Zusammensetzung der mineralischen Lösung, insbesondere die Gegenwart flüssiger Bestandteile, besonders Wasser bei unbekannter Konzentration (Druck); diese beiden Faktoren würden einen Einfluß von unbekannter Größe auf die Lage der

${ }^{1}$ Die Konstitution des Portlandzementes von P. H. BATES, ConcreteCement Age (Cement mill section) 2, 3 .

Z. anorg. Chem. Bd. 92 . 
verschiedenen Stabilitätsgebiete ausüben und könnten wohl ihre Zahl und ihren Charakter ändern. ${ }^{1}$ Aus diesem Grunde finden wir in der Natur Mineralien, die im wesentlichen aus $\mathrm{CaO}, \mathrm{Al}_{2} \mathrm{O}$ und $\mathrm{SiO}_{2}$ bestehen (z. B. Cyanit), die aber im Laboratorium nicht erhalten werden konnten; andererseits können Verbindungen dieser Oxyde, die bisher vom Petrographen nicht beobachtet worden sind, synthetisch hergestellt werden (z. B. $\mathrm{CaO} \cdot \mathrm{Al}_{2} \mathrm{O}_{3}$ ); aber aus diesen Umständen müssen wir den Schluß ziehen - in der Annahme, daß diese Verbindung in der Tat auf der Erde nicht vorkommt -, daB die Verbindung sich gebildet habe und später verschwunden ist, indem Verbindungen daraus entstanden sind, die unter den besonderen Verhältnissen größere Stabilität besitzen. In jedem Falle ist der erste Schritt bei einer vollständigen Untersuchung - die auch die Wirkungen des Druckes, der Gegenwart von Wasser usw. einschließen muß - eine sorgfältige Untersuchung der Beziehungen zwischen den reinen Komponenten und zwischen ihren Verbindungen, die sich unter den einfachsten Experimentalbedingungen bilden.

Einige mögliche Anwendungen auf geologische Probleme sollen in einer späteren Arbeit behandelt werden; im Augenblick möchten wir nur die Tatsache betonen, daß diese sehr wichtigen Unterschiede die Gültigkeit aller Folgerungen aus dem Verhalten und den Beziehungen der reinen trockenen Oxyde bei hohen Temperaturen und bei atmosphärischem Druck beeinflussen.

Die aus $\mathrm{CaO}, \mathrm{Al}_{2} \mathrm{O}_{3}$ und $\mathrm{SiO}_{2}$ bestehenden synthetischen Mineralien, die wir hergestellt haben, sind Quarz, Tridymit und Cristobalit $\left(\mathrm{SiO}_{2}\right)$; Korund $\left(\mathrm{Al}_{2} \mathrm{O}_{3}\right)$; Wollastonit $\left(\mathrm{CaO} . \mathrm{SiO}_{2}\right)$; Sillimanit $\left(\mathrm{Al}_{2} \mathrm{O}_{3} \cdot \mathrm{SiO}_{2}\right)$; Anorthit $\left(\mathrm{CaO} \cdot \mathrm{Al}_{2} \mathrm{O}_{3} \cdot 2 \mathrm{SiO}_{2}\right)$; diese alle haben dieselbe Zusammensetzung und praktisch dieselben optischen Eigenschaften wie die natürlichen Mineralien. Die Verbindung $2 \mathrm{CaO} \cdot \mathrm{Al}_{2} \mathrm{O}_{3} \cdot \mathrm{SiO}_{2}$ hat dieselben optischen Eigenschaften, aber nicht dieselbe Zusammensetzung, die man dem natürlichen Mineral Gehlenit zuschreibt; die Analyse dieses Minerals schwankt aber in so weiten Grenzen, daß man wohl ein Recht zu der Annahme hat, die wirkliche Zusammensetzung des natürlichen Gehlenits sei $2 \mathrm{CaO} \cdot \mathrm{Al}_{2} \mathrm{O}_{3} \cdot \mathrm{SiO}_{2}$. Die Verbindungen, welche gleichzeitig synthetische Mineralien sind, wurden in Fig. 19 unterstrichen; aus dieser sieht man, daß fünf Dreiecke rorhanden sind, in denen die allein vorkommenden Verbindungen synthetische Mineralien sind; jedes Dreieck ist das Stabilitätsgebiet

1 Eine Besprechung einiger dieser Punkte siehe bei JoHrston und NigGLI, Journ. Geology 21 (1913), 589-599. 
einer Gruppe von drei. In den Dreiecken, in denen $\mathrm{SiO}_{2}$ vorkommt, tritt es je nach der Temperatur entweder als Quarz oder als Tridymit oder als Cristobalit auf.

\section{Zusammenfassung.}

Auf den voraufgehenden Seiten ist ein Bericht über eine sorgfältige Untersuchung des Systemes $\mathrm{CaO}-\mathrm{Al}_{2} \mathrm{O}_{3}-\mathrm{SiO}_{2}$ gegeben, bei welcher etwa 7000 Erhitzungsversuche mit nachfolgenden optischen Prüfungen der Produkte ausgeführt wurden. Dies ternäre System erwies sich als kompliziert; das Gleichgewichtsdiagramm, das die Stabilitätsbeziehungen der Komponenten und Verbindungen bei atmosphärischem Druck darstellt, enthält nicht weniger als 14 getrennte Felder. Die Aufzeichnung der Grenzen der verschiedenen Felder war nur möglich, durch Anwendung der Phasenregel; das Ergebnis zeigt, daß dieses Prinzip ebenso auf die nur bei hohen Temperaturen flüssigen Oxydgemische anwendbar ist, wie auf die gewöhnlichen Lösungen.

Die Beziehungen zwischen den Komponenten und den verschiedẹen Verbindungen in den drei binären Systemen und im ternären System sind in einer Reihe von Temperatur-KonzentrationsDiagrammen dargestellt. Eine Liste dieser Verbindungen mit ihren Zusammensetzungen, Umwandlungs-, Schmelz- oder Dissoziationspunkten und optischen Eigenschaften sowie den Koordinaten (Temperatur und Zusammensetzung) der verschiedenen Quadrupel- und Quintupelpunkten ist beigefügt. Aus diesen Daten kann man mit Hilfe der Theorie der Kristallisationslinien, wie wir gezeigt haben, voraussagen, welche Stoffe sich aus einer langsam abkühlenden Lösung, die nur $\mathrm{CaO}, \mathrm{Al}_{2} \mathrm{O}_{3}$ und $\mathrm{SiO}_{2}$ enthält, abscheiden, in welcher Reihenfolge die Abscheidung stattfindet, und welches die entsprechenden Temperaturen sind.

Wenngleich das Diagramm mit den Endprodukten der Kristallisation die kontinuierliche Erreichung des Gleichgewichtes zur Voraussetzung hat, so kann man doch mit beträchtlicher Sicherheit das Endprodukt einer Reaktion in Gemischen der drei reinen Oxyde voraussagen, selbst wenn die Reaktion nicht vollständig verläuft. Dies ist der Fall beim Portlandzement-Klinker, der im wesentlichen ein Gemisch aus $3 \mathrm{CaO} . \mathrm{SiO}_{2}, 2 \mathrm{CaO} . \mathrm{SiO}_{2}, 3 \mathrm{CaO} . \mathrm{Al}_{2} \mathrm{O}_{3}$ mit etwas $5 \mathrm{CaO} .3 \mathrm{Al}_{2} \mathrm{O}_{3}$ und möglicherweise einer geringen Menge von freiem $\mathrm{CaO}$ ist, wenn man ihn in der gewöhnlichen Weise aus reinen Oxyden herstellt; dieser SchluB ist durch die Untersuchung des Bureau of Standards über technische Klinker bestätigt worden. 
Labelle 25. Optisch-kristallographische

\begin{tabular}{|c|c|c|c|c|c|c|}
\hline Zusammensetzung & $\begin{array}{l}\text { Krystall- } \\
\text { system }\end{array}$ & $\begin{array}{l}\text { Krystall- } \\
\text { tracht }\end{array}$ & Spaltbarkeit & Härte & $\begin{array}{l}\text { Ver- } \\
\text { länge- } \\
\text { rung }\end{array}$ & $\begin{array}{c}\text { Optische } \\
\text { Orientierung }\end{array}$ \\
\hline $\mathrm{CaO}$ & isometrisch & Würfel & $\begin{array}{c}\begin{array}{c}\text { vollkommen } \\
(\mathbf{1 0 0})\end{array} \\
\end{array}$ & $3-4$ & & \\
\hline $\begin{array}{c}\mathrm{Al}_{2} \mathrm{O}_{3} \\
\text { Korund }\end{array}$ & hexagonal & $\begin{array}{l}\text { dünne hexa- } \\
\text { gonal.Platten }\end{array}$ & & 9 & & $c=\alpha$ \\
\hline $\begin{array}{c}\mathrm{SiO}_{z} \\
\alpha-\text { Quarz }\end{array}$ & $\begin{array}{l}\text { hexagonal } \\
\text { trapezoedr.- } \\
\text { tetartoedr. } \\
\text { hexagonal }\end{array}$ & pyramidal & & 7 & $\gamma$ & $c=\gamma$ \\
\hline B-Quarz & $\begin{array}{l}\text { trapezoedr.- } \\
\text { hemiedr. }\end{array}$ & pyramidal & & & $\gamma$ & $c=\gamma$ \\
\hline$\alpha$-Tridymit & rhombisch & $\begin{array}{l}\text { dünne pseudo- } \\
\text { hex. Platten }\end{array}$ & & 6.5 & & \\
\hline$\beta-T r i d y m i t$ & & $\begin{array}{c}\text { hexagonale } \\
\text { Platten }\end{array}$ & & & & $c=\gamma$ \\
\hline$\alpha$-Cristobalit & $\begin{array}{c}\text { wahrscheinlich } \\
\text { tetragonal }\end{array}$ & Körner & & & & $c=\alpha$ \\
\hline$\beta$-Cristobalit & isometrisch & Körner & & & & \\
\hline $\begin{array}{l}\beta-\mathrm{CaO}, \mathrm{SiO}_{2} \\
\text { Wollastonit }\end{array}$ & monoklin & $\begin{array}{l}\text { Platten } \\
\text { Latten }\end{array}$ & $\begin{array}{l}g(100) \\
d(001)\end{array}$ & 5 & $\gamma$ & $\begin{array}{c}b=\beta \\
c: \alpha=+32\end{array}$ \\
\hline $\begin{array}{c}\alpha-\mathrm{CaO}_{-} \mathrm{SiO}_{2} \\
\text { Pseudo- } \\
\text { wollastonit }\end{array}$ & $\begin{array}{c}\text { monoklin } \\
\text { (pseado- } \\
\text { hexagonal) }\end{array}$ & Körner & & 5 & & \\
\hline $3 \mathrm{CaO} \cdot 2 \mathrm{SiO}_{2}$ & $\begin{array}{c}\text { wahrscheinlich } \\
\text { rhombisch }\end{array}$ & $\begin{array}{c}\text { unregelmäB } \\
\text { Körner }\end{array}$ & & & & \\
\hline$\gamma-2 \mathrm{CaO} \cdot \mathrm{SiO}_{3}$ & \begin{tabular}{|c|} 
wahrscheinlich \\
monoklin
\end{tabular} & prismatiseh & $\begin{array}{l}g(001) \\
g(100)\end{array}$ & & $\gamma$ & $\begin{array}{c}b=\beta \\
c: \gamma=3^{0}(?)\end{array}$ \\
\hline & $\begin{array}{c}\text { rhombisch oder } \\
\text { monoklin }\end{array}$ & $\begin{array}{l}\text { unregelmäB. } \\
\text { Körner,Prism. }\end{array}$ & schwach & $5-6$ & & \\
\hline $\begin{array}{l}\alpha-2 \mathrm{CaO} \cdot \mathrm{SiO}_{z} \\
\beta-2 \mathrm{CaO} \cdot \mathrm{SiO}_{2}\end{array}$ & $\begin{array}{c}\text { monoklin } \\
\text { oder triklin }\end{array}$ & $\begin{array}{c}\text { unregelmäB. } \\
\text { Körner } \\
\text { Körner } \\
\end{array}$ & & $5-6$ & & $c: \alpha=18^{\circ}$ \\
\hline $3 \mathrm{CaO} \cdot \mathrm{SiO}_{2}$ & monoklin (?) & Körner & & & & $c=\gamma$ \\
\hline $\begin{array}{c}\mathrm{Al}_{2} \mathrm{O}_{\mathrm{k}} \cdot \mathrm{SiO}_{2} \\
\text { Sillimanit }\end{array}$ & rhombiseh & $\begin{array}{l}\text { Fasern } \\
\text { Prismen }\end{array}$ & $g(100)$ & $6-7$ & $\gamma$ & $\begin{array}{l}b=\alpha \\
c=\gamma\end{array}$ \\
\hline $3 \mathrm{CaO} \cdot \mathrm{Al}_{2} \mathrm{O}_{3}$ & isometrisch & Körner & $\begin{array}{l}p(1111) \text { oder } \\
p(110)\end{array}$ & 6 & & \\
\hline 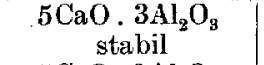 & isometrisch & Körner & $\begin{array}{c}\text { schwach } \\
\text { prismatisch }\end{array}$ & 5 & & \\
\hline $\begin{array}{c}5 \mathrm{CaO} .3 \mathrm{Al}_{2} \mathrm{O}_{3} \\
\text { instabil }\end{array}$ & $\begin{array}{c}\text { wahrscheinlich } \\
\text { rhombisch }\end{array}$ & $\begin{array}{l}\text { Fasern } \\
\text { Prismen }\end{array}$ & 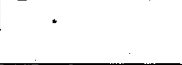 & 5 & $\alpha$ & \\
\hline $\mathrm{CaO} \cdot \mathrm{Al}_{2} \mathrm{O}_{3}$ & $\begin{array}{c}\text { wahrscheinlioh } \\
\text { monoklin }\end{array}$ & $\begin{array}{c}\text { unregelmäß. } \\
\text { Körn. Fasern }\end{array}$ & prismatisch & 6.5 & & \\
\hline $\begin{array}{c}3 \mathrm{CaO} .5 \mathrm{Al}_{2} \mathrm{O}_{3} \\
\text { stabil }\end{array}$ & tetragınal & Körner & & 6.5 & $\gamma$ & $c=\gamma$ \\
\hline $\begin{array}{c}3 \mathrm{CaO} .5 \mathrm{Al}_{2} \mathrm{O}_{3} \\
\text { instabil }\end{array}$ & $\begin{array}{c}\text { wahrscheinlich } \\
\text { rhombisch }\end{array}$ & $\begin{array}{c}\text { Faserm } \\
\text { Prismen }\end{array}$ & & 5.5 & $\gamma$ & \\
\hline $\begin{array}{c}\mathrm{CaO} . \mathrm{Al}_{2} \mathrm{O}_{3} \cdot 2 \mathrm{SiO}_{2} \\
\text { Anorthit }\end{array}$ & triklin & $\begin{array}{l}\text { Latten } \\
\text { Platten } \\
\end{array}$ & $\begin{array}{l}g(001) \\
g(010)\end{array}$ & 6 & & $\begin{array}{c}\alpha: \alpha^{\prime} \text { auf } \\
001=-35^{\circ}\end{array}$ \\
\hline $\begin{array}{c}\mathrm{CaO} \mathrm{Al}_{3} \mathrm{O}_{3} \cdot \mathrm{SiO}_{2} \\
\text { Gehlenit }\end{array}$ & tetragonal & Körner & $d(001)$ & 6 & & $c=\boldsymbol{x}$ \\
\hline $3 \mathrm{CaO}, \mathrm{Al}_{2} \mathrm{O}_{3}, \mathrm{SiO}_{2}$ & rhombisch (?) & Fasern & & & $\gamma$ & \\
\hline
\end{tabular}


Eigenschaften der Verbindungen.

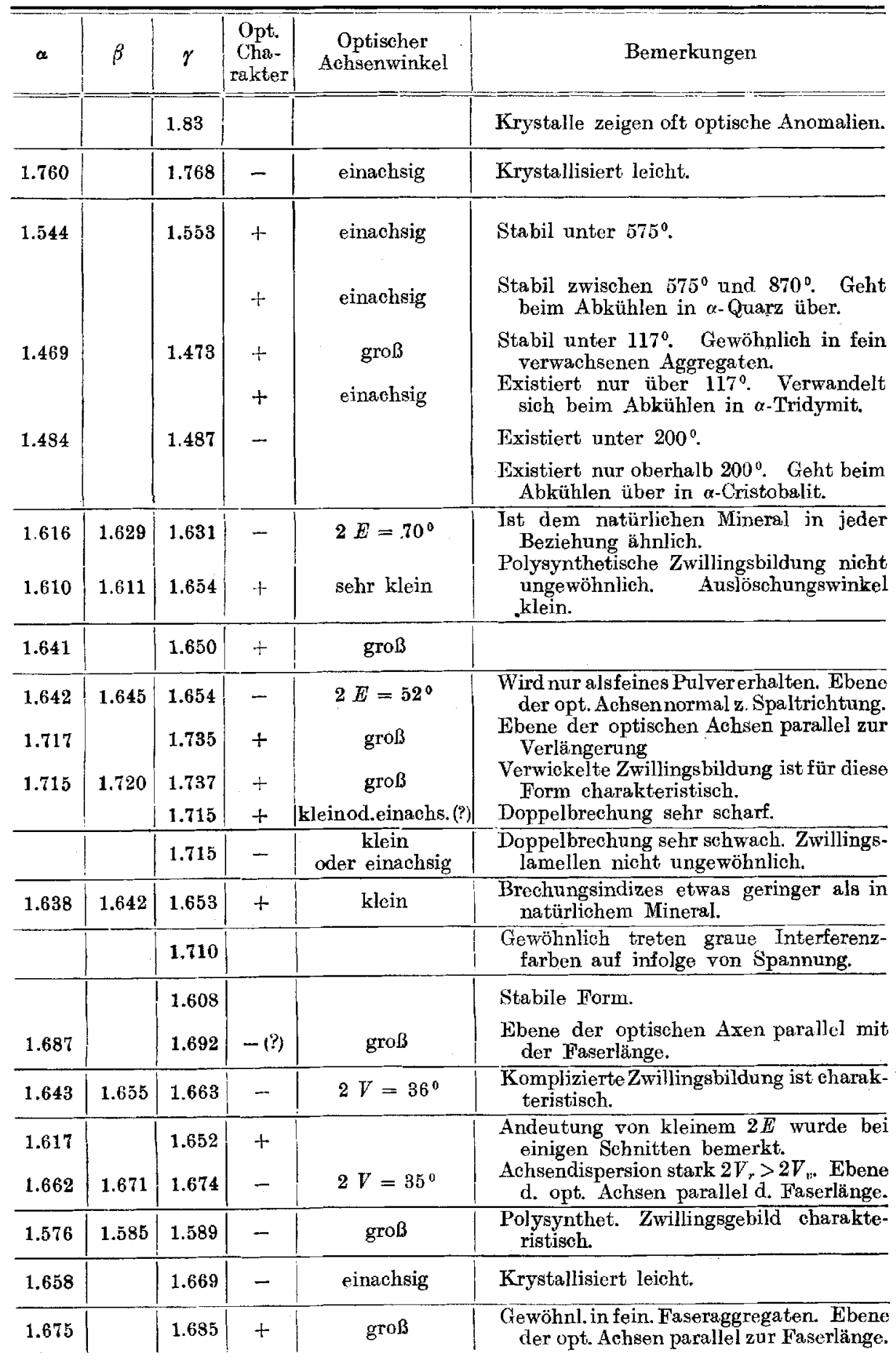


Tabelle 26.

A. Verbindungen. - Umwandlungspunkte.

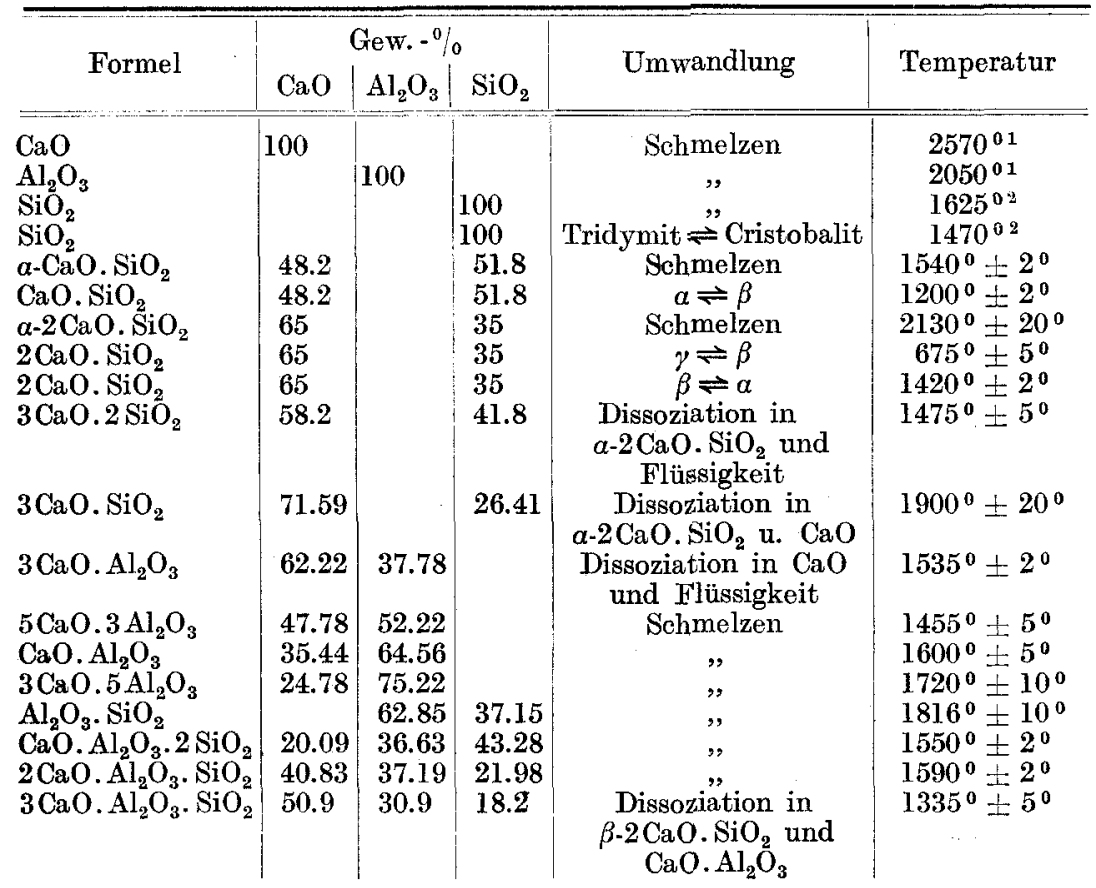

B. Binäre Eutektika - Schmelzpunkte.

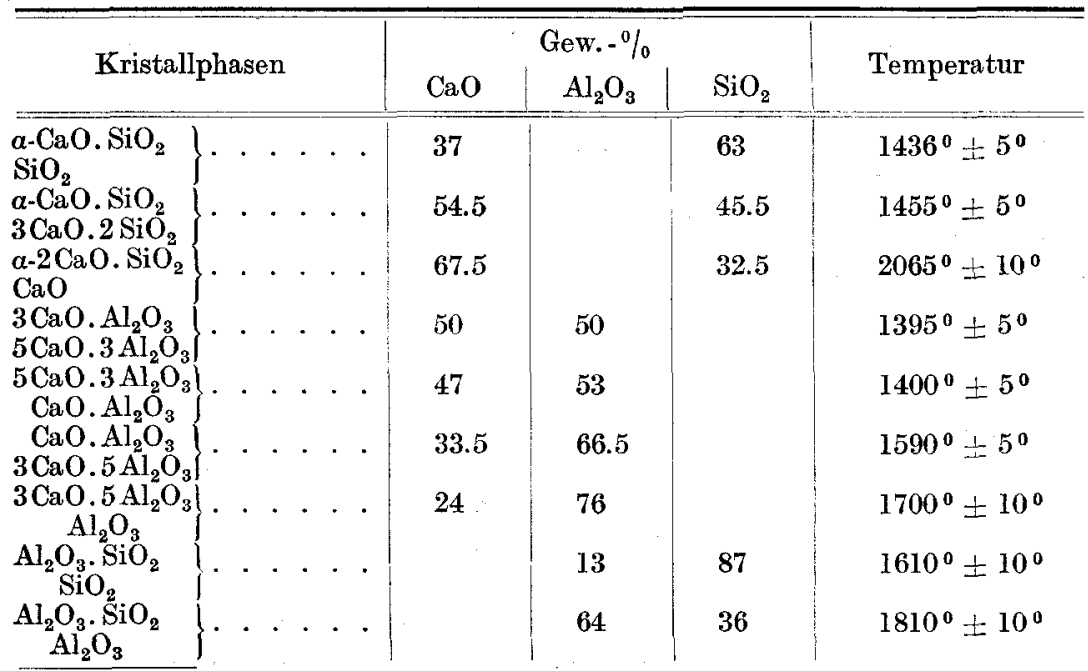

1 Kanolt, J., Wash. Acad. Sci. 3 (1913), 315.

2 Fenner, Am. J. Sci. (4) 37 (1913), 331-384. 
Ternäres System: Calciumoxyd-Aluminiumoxyd-Silicium-2•oxyd. 295

B. Binäre Eutektika - Schmelzpunkte (Fortsetzung).

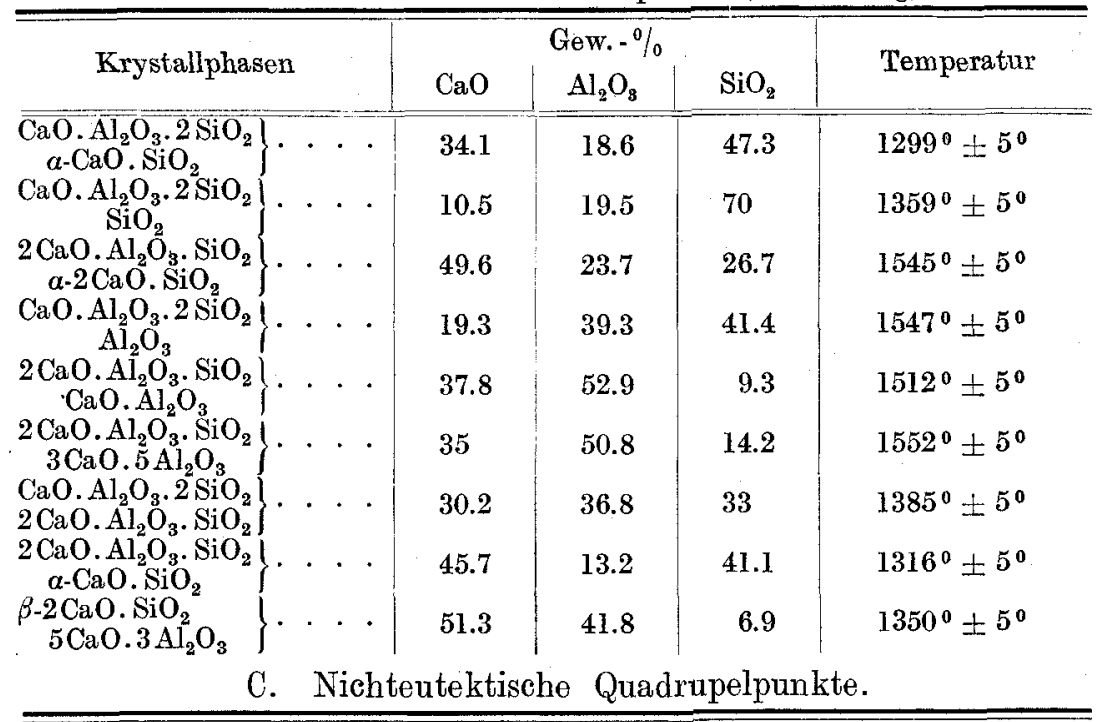

\begin{tabular}{|c|c|c|c|c|}
\hline Kristallphasen & $\mathrm{CaO}$ & $\begin{array}{l}\text { Gew. }-\% \\
\mathrm{Al}_{2} \mathrm{O}_{3}\end{array}$ & $\mathrm{SiO}_{2}$ & Temperatur \\
\hline $\left.\begin{array}{c}3 \mathrm{CaO} .2 \mathrm{SiO}_{2} \\
\mathrm{a}-2 \mathrm{CaO} . \mathrm{SiO}_{2} \\
\mathrm{CaO} \\
3 \mathrm{CaO} . \mathrm{Al}_{2} \mathrm{O}_{3}\end{array}\right\} \cdots$ & $\begin{array}{l}55.5 \\
57\end{array}$ & 43 & 44.5 & $\begin{array}{l}1475^{\circ} \pm 5^{0} \\
1535^{\circ} \pm 2^{\circ}\end{array}$ \\
\hline
\end{tabular}

\begin{tabular}{|c|c|c|c|c|}
\hline \multirow{2}{*}{ Kristallphasen } & \multicolumn{3}{|c|}{ Gew. $\%$} & \multirow{2}{*}{ Temperatur } \\
\hline & $\mathrm{CaO}$ & $\mathrm{Al}_{2} \mathrm{O}_{3}$ & $\mathrm{SiO}_{2}$ & \\
\hline $\begin{array}{c}\mathrm{CaO} \cdot \mathrm{Al}_{2} \mathrm{O}_{3} \cdot 2 \mathrm{SiO}_{2} \\
\mathrm{ilO}_{2} \\
a-\mathrm{CaO} . \mathrm{SiO}_{2}\end{array}$ & 23.25 & I4.75 & 62 & $1165^{\circ} \pm 5^{\circ}$ \\
\hline $\begin{array}{c}\mathrm{CaO} \cdot \mathrm{Al}_{2} \mathrm{O}_{3} \cdot 2 \mathrm{SiO}_{2} \\
\mathrm{Al}_{2} \mathrm{O}_{3} \cdot \mathrm{SiO}_{2} \\
\mathrm{SiO}_{2}\end{array}$ & 9.8 & 19.8 & 70.4 & $1345^{0} \pm 5^{0}$ \\
\hline $\begin{array}{c}2 \mathrm{CaO} . \mathrm{Al}_{2} \mathrm{O}_{3} \cdot \mathrm{SiO}_{2} \\
3 \mathrm{CaO} \cdot 2 \mathrm{SiO}_{2} \\
\alpha-\mathrm{CaO} \cdot \mathrm{SiO}_{2}\end{array}$ & 47.2 & 11.8 & 41 & $1310^{\circ} \pm 5^{0}$ \\
\hline $\begin{array}{c}2 \mathrm{CaO} . \mathrm{Al}_{2} \mathrm{O}_{3} \cdot \mathrm{SiO}_{2} \\
\mathrm{CaO} . \mathrm{Al}_{2} \mathrm{O}_{3} \\
3 \mathrm{CaO} .5 \mathrm{Al}_{2} \mathrm{O}_{3}\end{array}$ & 37.5 & 53.25 & 9.25 & $1505^{0} \pm 5^{0}$ \\
\hline $\begin{array}{c}\mathrm{CaO} \cdot \mathrm{Al}_{2} \mathrm{O}_{3} \cdot 2 \mathrm{SiO}_{2} \\
2 \mathrm{CaO} \cdot \mathrm{Al}_{2} \mathrm{O}_{3} \cdot \mathrm{SiO}_{2} \\
a \text {-CaO. } \mathrm{SiO}_{2}\end{array}$ & 38 & 20 & 42 & $1265^{\circ} \pm 5^{0}$ \\
\hline $\begin{array}{c}\mathrm{CaO} \cdot \mathrm{Al}_{2} \mathrm{O}_{3} \cdot 2 \mathrm{SiO}_{2} \\
2 \mathrm{CaO} \cdot \mathrm{Al}_{2} \mathrm{O}_{3} \cdot \mathrm{SiO}_{2} \\
\mathrm{Al}_{2} \mathrm{O}_{3}\end{array}$ & 29.2 & 39 & 31.8 & $1380^{\circ} \pm \mathbf{5}^{0}$ \\
\hline $\begin{array}{c}\beta-2 \mathrm{CaO} \cdot \mathrm{SiO}_{2} \\
\mathrm{CaO} \cdot \mathrm{Al}_{2} \mathrm{O}_{3} \\
5 \mathrm{CaO} .3 \mathrm{Al}_{2} \mathrm{O}_{3}\end{array}$ & 49.5 & 43.7 & 6.8 & $1335^{0} \pm 5^{0}$ \\
\hline $\begin{array}{l}\beta-2 \mathrm{CaO} \cdot \mathrm{SiO}_{2} \\
3 \mathrm{CaO} . \mathrm{Al}_{2} \mathrm{O}_{3} \\
5 \mathrm{CaO} .3 \mathrm{Al}_{2} \mathrm{O}_{3}\end{array}$ & 52 & 41.2 & 6.8 & $1335^{\circ} \pm 5^{0}$ \\
\hline
\end{tabular}


296 G. A. Rankin. Das ternäre System: Calciumoxyd-Aluminiumoxyd usw.

E. Nichteutektische Quintupelpunkte.

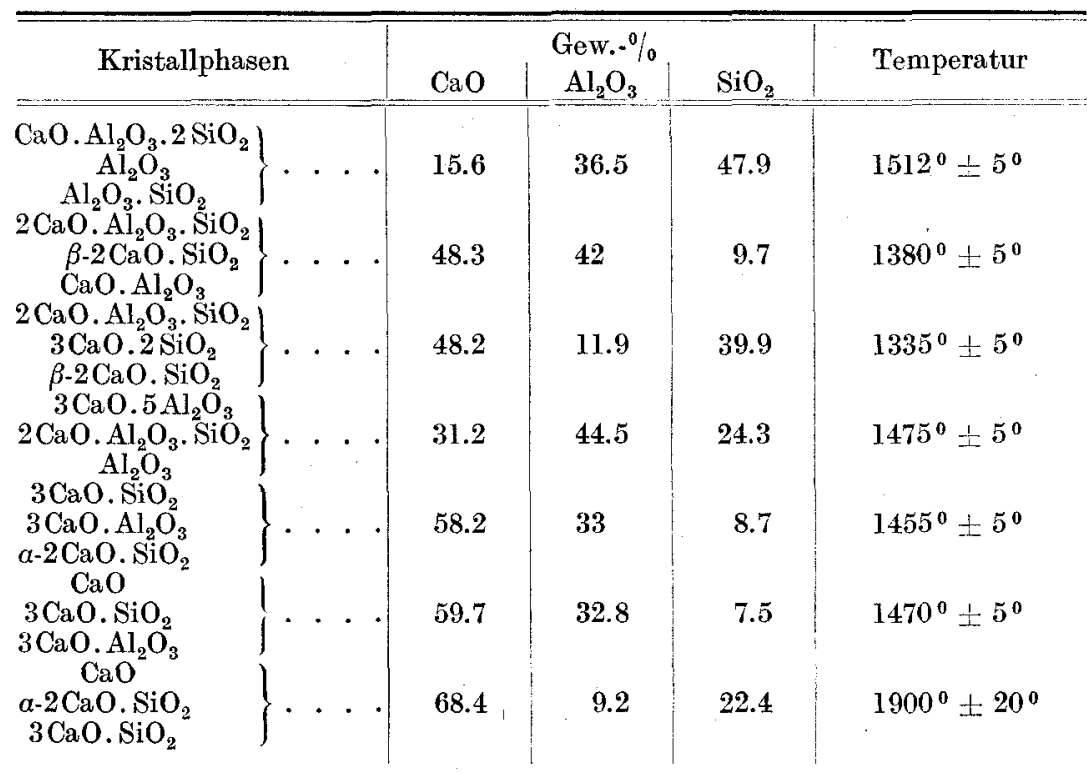

Beim Abschluß dieses Berichtes über eine lange und bisweilen recht schwierige Untersuchung ist es den Verfassern ein besonderes Vergnügen, Herrn Professor W. D. Bancroft für viele Ratschläge ihren Dank auszusprechen und ebenso ihren Herren Kollegen Dr. John Johnston, E. S. Shepherd und I. B. Ferguson für ihre freundliche vielseitige Unterstützung.

Geophysical Laboratory, Carnegie Inst. of Washington. Washington, D. C.

Bei der Redaktion eingegangen am 6. Februar 1915. 